\title{
The characteristic class and ramification of an $\ell$-adic étale sheaf
}

\author{
Ahmed Abbes and Takeshi Saito \\ September 18, 2018
}

Dedicated to Luc Illusie, with admiration

\begin{abstract}
We introduce the characteristic class of an $\ell$-adic étale sheaf using a cohomological pairing due to Verdier (SGA5). As a consequence of the Lefschetz-Verdier trace formula, its trace computes the Euler-Poincaré characteristic of the sheaf. We compare the characteristic class to two other invariants arising from ramification theory. One is the Swan class of Kato-Saito [16] and the other is the 0 -cycle class defined by Kato for rank 1 sheaves in [15].
\end{abstract}

Let $k$ be a perfect field, $\ell$ be a prime number invertible in $k$ and $\Lambda$ be a finite extension of either $\mathbb{F}_{\ell}$ or $\mathbb{Q}_{\ell}$. Let $X$ be a separated $k$-scheme of finite type. Let $f: X \rightarrow$ Spec $k$ denote the structural morphism and put $\mathcal{K}_{X}=R f^{!} \Lambda$. For an étale sheaf $\mathcal{F}$ of $\Lambda$-modules on $X$, its characteristic class is defined as follows. We put $\mathcal{H}=$ $R \mathcal{H o m}\left(\mathrm{pr}_{2}^{*} \mathcal{F}, R \mathrm{pr}_{1}^{!} \mathcal{F}\right)$ and $\mathcal{H}^{*}=R \mathcal{H}$ om $\left(\operatorname{pr}_{1}^{*} \mathcal{F}, R \operatorname{pr}_{2}^{!} \mathcal{F}\right)$ on $X \times X$ and let $\Delta=X \subset X \times$ $X$ denote the diagonal. Then, we have $1 \in \operatorname{End}(\mathcal{F})=H_{\Delta}^{0}(X \times X, \mathcal{H})=H_{\Delta}^{0}\left(X \times X, \mathcal{H}^{*}\right)$. The natural pairing $\mathcal{H} \otimes \mathcal{H}^{*} \rightarrow \mathcal{K}_{X \times X}$ induces a pairing $\langle\rangle:, H_{\Delta}^{0}(X \times X, \mathcal{H}) \otimes H_{\Delta}^{0}(X \times$ $\left.X, \mathcal{H}^{*}\right) \rightarrow H_{\Delta}^{0}\left(X \times X, \mathcal{K}_{X \times X}\right)=H^{0}\left(X, \mathcal{K}_{X}\right)$. We define the characteristic class of $\mathcal{F}$, denoted $C(\mathcal{F})$, to be the pairing $\langle 1,1\rangle \in H^{0}\left(X, \mathcal{K}_{X}\right)$. If $X$ is smooth of dimension $d$, we have $C(\mathcal{F}) \in H^{2 d}(X, \Lambda(d))$. By the Lefschetz-Verdier trace formula 11] Théorème 4.4, the trace $\operatorname{Tr} C(\mathcal{F})$ gives the Euler-Poincaré characteristic $\chi\left(X_{\bar{k}}, \mathcal{F}\right)$ if $X$ is proper, where $\bar{k}$ denotes a separable closure of $k$.

By devissage, computations of the characteristic classes are reduced to a computation of $C(j ! \mathcal{F})$ where $j: U \rightarrow X$ is an open immersion, $U$ is smooth and $\mathcal{F}$ is a smooth sheaf on $U$. In this paper, we compute the characteristic class of $C(j ! \mathcal{F})$ or rather the difference $C(j ! \mathcal{F})$-rank $\mathcal{F} \cdot C\left(j_{!} \Lambda\right)$ in terms of the ramification of $\mathcal{F}$ along the boundary $X \backslash U$. More precisely, we prove that $C\left(j_{!} \mathcal{F}\right)-\operatorname{rank} \mathcal{F} \cdot C\left(j_{!} \Lambda\right)$ is equal to the following two invariants, under certain assumptions. One is the Swan class $\operatorname{Sw}(\mathcal{F})$ of $\mathcal{F}$ defined in [16]. The other is the 0 -cycle class $c_{\mathcal{F}}$ defined by Kato [15] in rank 1 case. We also define a localization of the difference $C\left(j_{!} \mathcal{F}\right)-\operatorname{rank} \mathcal{F} \cdot C\left(j_{!} \Lambda\right)$ in $H_{X \backslash U}^{0}\left(X, \mathcal{K}_{X}\right)$ as a cohomology class with support on the boundary. 
The relation with the Swan class is a refinement of the generalized GrothendieckOgg-Shafarevich formula proved in [16]. The proof uses a finite étale covering of $U$ trivializing the reduction modulo $\ell$ of the $\ell$-adic sheaf $\mathcal{F}$. It is similar to that of the generalized Grothendieck-Ogg-Shafarevich formula in loc. cit. The key ingredients in the proof are the compatibility of the characteristic class with pull-back and an explicit computation of the characteristic class in the tamely ramified case. This argument works in arbitrary dimension and in arbitrary rank, under a certain mild assumption that the sheaf is "potentially of Kummer type" (see Definition 3.1.1). However, since we use the pull-back to a covering, we can not avoid a denominator.

The relation with the other invariant $c_{\mathcal{F}}$ in rank 1 case is proved using a blow-up at the ramification locus in the diagonal $X \subset X \times X$. This argument does not involve a denominator and we get an integral result. However, even to formulate the statement at least for the moment, we need to assume that the sheaf has rank one. Using this approach, we obtain a new proof of the Grothendieck-Ogg-Shafarevich formula for curves without using a covering trivializing the sheaf or the Weil formula. A crucial fact, used in the proof, is the following geometric interpretation of the ramification theory for Artin-Schreier-Witt characters of Kato [14, proved in [2].

Let $(X \times X)^{\prime} \rightarrow X \times X$ be the log blow-up and $X \rightarrow(X \times X)^{\prime}$ be the log diagonal map. We consider the blow-up $(X \times X)^{\prime \prime} \rightarrow(X \times X)^{\prime}$ at the Swan divisor $D_{\mathcal{F}} \subset X$ regarded as a closed subscheme of $(X \times X)^{\prime}$ by the log diagonal map. Then, the sheaf $\mathcal{H} o m\left(\operatorname{pr}_{2}^{*} \mathcal{F}, \operatorname{pr}_{1}^{*} \mathcal{F}\right)$ on $U \times U$ is unramified along the exceptional divisor of $(X \times X)^{\prime \prime}$. Further the restriction of a smooth extension of $\mathcal{H o m}\left(\operatorname{pr}_{2}^{*} \mathcal{F}, \operatorname{pr}_{1}^{*} \mathcal{F}\right)$ on the exceptional divisor is the Artin-Schreier sheaf defined by the refined Swan conductor. This interpretation means that one can kill the ramification of a sheaf not only by a ramified covering but also by blowing-up the diagonal on the ramification locus. More quantitatively, the Swan conductor measures the necessary blow-up to kill the ramification. This fits nicely with the ramification theory in 11. The authors expect that this argument should work with arbitrary rank (cf. [2]).

The paper is organized as follows. In Section 1, we recall some sorites on cohomological correspondences and the Verdier pairing, and we give some complements. In Section 2, we define the characteristic class of a cohomological correspondence. Proposition (2.1.12) gives a recipe to compute it. It plays a crucial role in this paper. Then we compute the characterstic class under some tameness condition. In Section 3, we introduce the notion of a sheaf of Kummer type, and recall the definition of the Swan class of Kato-Saito [16]. For a sheaf potentially of Kummer type, we prove the main formula of this paper relating its characteristic class and its Swan class. In Section 4, we study sheaves of rank one. We recall the ramification theory of Artin-Schreier-Witt characters in [14]. Then, we prove an integral relation between the characteristic class and the 0 -cycle class $c_{\mathcal{F}}$ for a rank one sheaf, by blowing-up the diagonal. In Section 5 , we define a cohomology class $C_{X \backslash U}^{0}(\mathcal{F}) \in H_{X \backslash U}^{0}\left(X, \mathcal{K}_{X}\right)$ with support on the boundary and show that it is a refinement of the difference $C(j ! \mathcal{F})-\operatorname{rank} \mathcal{F} \cdot C(j ! \Lambda)$.

The authors thank Luc Illusie and Kazuya Kato for inspiring discussions. They thank Illusie for pointing out an error in Lemma2.1.3 in an earlier version and informing 
the reference 9].

\section{Notation}

In this paper, $k$ denotes a field. In sections 3 and 4 , we will assume $k$ perfect. Schemes over $k$ are assumed separated and of finite type. Thus the diagonal map $\delta: X=\Delta_{X} \rightarrow X \times X$ is a closed immersion. For a divisor with simple normal crossings of a smooth scheme over $k$, we assume that the irreducible components and their intersections are also smooth over $k$.

The letter $\ell$ denotes a prime number invertible in $k$ and $\Lambda$ denotes a finite com-

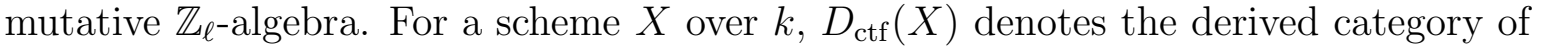
complexes of $\Lambda$-modules of finite tor-dimension on the étale site of $X$ with constructible cohomology 7. 4.6. We omit to write $R$ or $L$ to denote the derived functors unless otherwise stated explicitly or for $R \mathcal{H}$ om. Let $\mathcal{K}_{X}$ denote $f^{!} \Lambda$ where $f: X \rightarrow$ Spec $k$ is the structure map and let $\mathbf{D}$ denote the functor $R \mathcal{H}$ om $\left(, \mathcal{K}_{X}\right)$. For objects $\mathcal{F}$ and $\mathcal{G}$ of $D_{\text {ctf }}(X)$ and $D_{\text {ctf }}(Y)$ on schemes $X$ and $Y$ over $k, \mathcal{F} \otimes \mathcal{G}$ denotes $\operatorname{pr}_{1}^{*} \mathcal{F} \otimes \operatorname{pr}_{2}^{*} \mathcal{G}$ on $X \times Y$.

\section{Preliminaries on cohomological correspondences.}

We recall some generalities on étale cohomology and constructions in [1].

\subsection{Review on base change maps, cycle class maps etc.}

Let

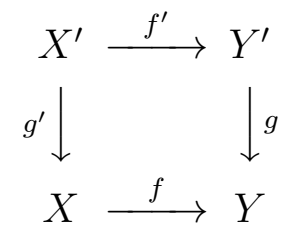

be a commutative diagram of schemes over $k$. We have base change morphisms of functors

$$
\begin{aligned}
g^{*} f_{*} & \rightarrow f_{*}^{\prime} g^{*}, \\
f_{!}^{\prime} g^{\prime !} & \rightarrow g^{!} f_{!}
\end{aligned}
$$

([5] 4.1.5, [6 3.1.13.2). If $f$ is proper, if $g$ is an open immersion and if the square is cartesian, the maps (1.1) and (1.2) are isomorphisms and are inverse to each other. If $g^{\prime}$ is proper, the base change map

$$
g^{*} f_{!} \rightarrow f_{!}^{\prime} g^{*}
$$


is similarly defined as the adjoint of the composition $f_{!} \rightarrow f_{!} g_{*}^{\prime} g^{\prime *}=f_{!} g_{!}^{\prime} g^{\prime *} \rightarrow g_{!} f_{!}^{\prime} g^{\prime *} \rightarrow$ $g_{*} f_{!}^{\prime} g^{\prime *}$. The base change maps (1.1) and (1.3) form a commutative diagram

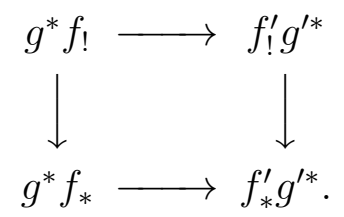

For a commutative diagram

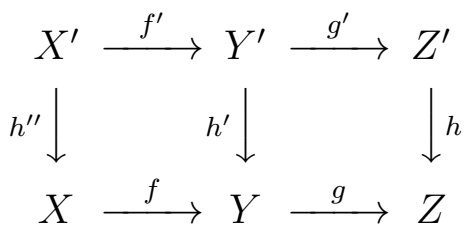

of schemes over $k$, the base change morphisms (1.2) are transitive. Namely, we have a commutative diagram

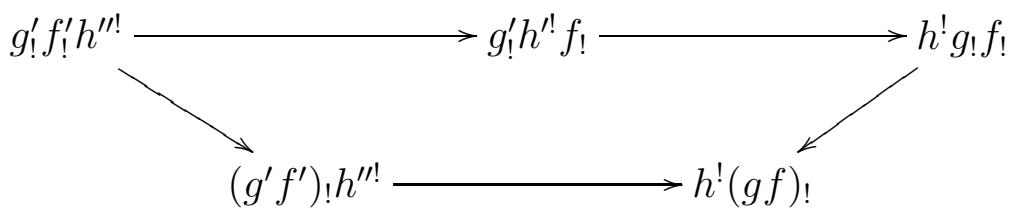

where the slant arrows are induced by the composition isomorphisms. We also have a similar commutative diagram for base change morphisms (1.1). If $h^{\prime}$ and $h^{\prime \prime}$ are proper, we have a similar commutative diagram for (1.3).

For a morphism $f: X \rightarrow Y$ of schemes over $k$ and objects $\mathcal{F}$ and $\mathcal{G}$ of $D_{\text {ctf }}(Y)$, we recall the definition of the canonical isomorphism

$$
R \mathcal{H o m}\left(f^{*} \mathcal{F}, f^{!} \mathcal{G}\right) \rightarrow f^{!} R \mathcal{H} \operatorname{om}(\mathcal{F}, \mathcal{G})
$$

(6] 3.1.12.2). A canonical map $\mathcal{F} \otimes f_{!} R \mathcal{H} \operatorname{com}\left(f^{*} \mathcal{F}, f^{!} \mathcal{G}\right) \rightarrow f_{!}\left(f^{*} \mathcal{F} \otimes R \mathcal{H o m}\left(f^{*} \mathcal{F}, f^{!} \mathcal{G}\right)\right)$ is defined in $\left(\left[\right.\right.$ [ 3.1.11.2). By composing $f_{!}\left(f^{*} \mathcal{F} \otimes R \mathcal{H}\right.$ om $\left.\left(f^{*} \mathcal{F}, f^{!} \mathcal{G}\right)\right) \rightarrow f_{!} f^{!} \mathcal{G} \rightarrow \mathcal{G}$, we obtain $\mathcal{F} \otimes f_{!} R \mathcal{H} o m\left(f^{*} \mathcal{F}, f^{!} \mathcal{G}\right) \rightarrow \mathcal{G}$. This induces the map (1.5) by adjunction. If $\mathcal{F}$ and $\mathcal{G}$ are objects of the filtered derived category [12 Chap. V $\S \S 1-3$, the isomorphism (1.5) is an isomorphism in the filtered derived category. Recall that an object of $D F_{\text {ctf }}(X)$ is a complex $\mathcal{F}$ of $\Lambda$-modules on the étale site of $X$ with finite filtration $F$. such that $G r_{\bullet}^{F} \mathcal{F}$ is an object of $D_{\text {ctf }}(X)$. In particular, we have an isomorphism

$$
\operatorname{Hom}_{D F_{\mathrm{ctf}}(X)}\left(f^{*} \mathcal{F}, f^{!} \mathcal{G}\right) \rightarrow \Gamma\left(X, f^{!} F_{0} R \mathcal{H} \operatorname{om}(\mathcal{F}, \mathcal{G})\right) .
$$

For a morphism $f: X \rightarrow Y$ of schemes over $k$ and objects $\mathcal{F}$ and $\mathcal{G}$ of $D_{\text {ctf }}(Y)$, a canonical map

$$
f^{*} \mathcal{F} \otimes f^{!} \mathcal{G} \rightarrow f^{!}(\mathcal{F} \otimes \mathcal{G})
$$


is defined as follows. We have the canonical isomorphism $f_{!}\left(f^{*} \mathcal{F} \otimes f^{!} \mathcal{G}\right) \rightarrow \mathcal{F} \otimes f_{!} f^{!} \mathcal{G}$ of the projection formula ([5] 5.2.9). By composing with the adjunction map $f_{!} f^{!} \mathcal{G} \rightarrow \mathcal{G}$, we obtain a map $f_{!}\left(f^{*} \mathcal{F} \otimes f^{!} \mathcal{G}\right) \rightarrow \mathcal{F} \otimes \mathcal{G}$. Thus, the canonical map (1.7) is defined by taking the adjoint.

For a flat morphism $f: X \rightarrow Y$ of fiber dimension $d$ of schemes over $k$, the trace map $f_{!} \Lambda(d)[2 d] \rightarrow \Lambda$ is defined ([6] 2.9) and the canonical class map

$$
\Lambda(d)[2 d] \rightarrow R f^{!} \Lambda
$$

is defined as its adjoint ([6] 3.2.3). If $f$ is smooth, the map (1.8) is an isomorphism ([6] $3.2 .5)$.

Let $f: X \rightarrow Y$ be a morphism of smooth schemes of the same dimension $n$ over $k$. The map $f$ is the composition $\operatorname{pr}_{2} \circ \gamma$ of the graph map $\gamma: X \rightarrow X \times Y$ and the second projection $\mathrm{pr}_{2}: X \times Y \rightarrow Y$. Since $\gamma$ is a section of the first projection $\operatorname{pr}_{1}: X \times Y \rightarrow$ $X$ and the projections are smooth of relative dimension $n$, a canonical isomorphism $\Lambda \rightarrow f^{!} \Lambda$ is defined as the composition $\Lambda \rightarrow \gamma^{!} \operatorname{pr}_{1}^{!} \Lambda \stackrel{\sim}{\leftarrow} \gamma^{!} \Lambda(n)[2 n] \rightarrow \gamma^{!} \operatorname{pr}_{2}^{!} \Lambda \rightarrow f^{!} \Lambda$. Thus the canonical map (1.7) for $\mathcal{G}=\Lambda$ induces a functor

$$
f^{*} \rightarrow f^{!}
$$

We recall the cycle class map. Let $C$ be a scheme over $k$ and $d \geq 0$ be an integer. For an integral closed subscheme $V \subset C$ of dimension $d$, the canonical class map $\Lambda(d)[2 d] \rightarrow \mathcal{K}_{V}$ gives the cycle class $[V] \in H^{0}\left(V, \mathcal{K}_{V}(-d)[-2 d]\right)=H_{V}^{-2 d}\left(C, \mathcal{K}_{C}(-d)\right)$. The cycle classes define a map cl: $Z_{d}(C) \rightarrow H^{-2 d}\left(C, \mathcal{K}_{C}(-d)\right)$ where $Z_{d}(C)$ denotes the free abelian group generated by the integral closed subschemes of $C$ of dimension $d$. The cycle class map $Z_{d}(C) \rightarrow H^{-2 d}\left(C, \mathcal{K}_{C}(-d)\right)$ factors through the quotient by rational equivalence and induces a map

$$
\mathrm{cl}_{C}: C H_{d}(C) \longrightarrow H^{-2 d}\left(C, \mathcal{K}_{C}(-d)\right)
$$

If $C$ is a closed subscheme of a smooth scheme $X$ of dimension $d+c$, the target $H^{-2 d}\left(C, \mathcal{K}_{C}(-d)\right)$ is identified with $H_{C}^{-2 d}\left(X, \mathcal{K}_{X}(-d)\right)=H_{C}^{2 c}(X, \Lambda(c))$. The cycle class map and the intersection product are compatible in the following sense. Let $X$ and $Y$ be smooth schemes of dimension $d+c$ and $e+c$ over $k$ and $f: X \rightarrow Y$ be a morphism over $k$. Let $C \subset X$ and $D \subset Y$ be closed subschemes satisfying $f^{-1}(D) \subset C$ set-theoretically. Then the diagram

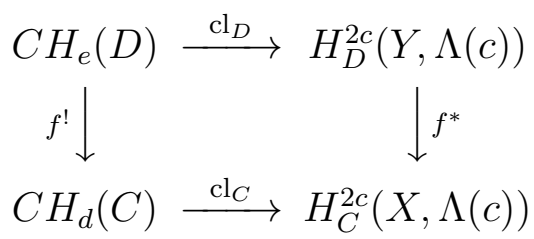

is commutative by [16] Lemma 2.1.2. The left vertical arrow $f^{!}: C H_{e}(D) \rightarrow C H_{d}(C)$ denotes the Gysin map in the intersection theory [10]. The commutative diagram (1.11) for the diagonal map $\delta: X \rightarrow Y=X \times X$ implies the compatibility

$$
\operatorname{cl}(V) \cup \operatorname{cl}(W)=\operatorname{cl}(V, W)_{X}
$$


in $H_{V \cap W}^{2(c+d)}(X, \Lambda(c+d))$ of the cup-product and the intersection theory for closed subschemes $V$ and $W$ in $X$ of codimensions $c$ and $d$.

\subsection{Cohomological correspondences.}

We recall definitions on cohomological correspondences following [11. In order to remain compatible with the convention in [5] 1.1.10 (ii), we have chosen to switch the factors in [11] and consider a cohomological correspondence as a map from the second factor to the first factor.

Definition 1.2.1 Let $X$ and $Y$ be schemes over $k$ and $\mathcal{F}$ and $\mathcal{G}$ be objects of $D_{\text {ctf }}(X)$ and of $D_{\mathrm{ctf}}(Y)$ respectively. We call a correspondence between $X$ and $Y$ a scheme $C$ over $k$ and morphisms $c_{1}: C \rightarrow X$ and $c_{2}: C \rightarrow Y$ over $k$. We put $c=\left(c_{1}, c_{2}\right): C \rightarrow$ $X \times Y$ the corresponding morphism. We call a morphism $u: c_{2}^{*} \mathcal{G} \rightarrow c_{1}^{!} \mathcal{F}$ a cohomological correspondence from $\mathcal{G}$ to $\mathcal{F}$ on $C$.

The definition here is slightly different from that given in [1] 3.2 if $c: C \rightarrow X \times Y$ is not proper. If $c: C \rightarrow X \times Y$ is the graph map $\gamma: X \rightarrow X \times Y$ of a map $f: X \rightarrow Y$, a cohomological correspondence from $\mathcal{G}$ to $\mathcal{F}$ on $C$ is nothing but a map $f^{*} \mathcal{G} \rightarrow \mathcal{F}$.

By the isomorphism (1.5)

$$
c^{!} R \mathcal{H o m}\left(\operatorname{pr}_{2}^{*} \mathcal{G}, \operatorname{pr}_{1}^{!} \mathcal{F}\right) \rightarrow R \mathcal{H o m}\left(c_{2}^{*} \mathcal{G}, c_{1}^{!} \mathcal{F}\right)
$$

and by the adjunction, the following three notions are equivalent:

(1) a cohomological correspondence $u: c_{2}^{*} \mathcal{G} \rightarrow c_{1}^{!} \mathcal{F}$ on $C$.

(2) a section of $c^{!} R \mathcal{H}$ om $\left(\operatorname{pr}_{2}^{*} \mathcal{G}, \operatorname{pr}_{1}^{!} \mathcal{F}\right)$ on $C$, or equivalently a map $\Lambda_{C} \rightarrow c^{!} R \mathcal{H}$ om $\left(\operatorname{pr}_{2}^{*} \mathcal{G}, \operatorname{pr}_{1}^{!} \mathcal{F}\right)$.

(3) a map $c_{!} \Lambda_{C} \rightarrow R \mathcal{H o m}\left(\operatorname{pr}_{2}^{*} \mathcal{G}, \operatorname{pr}_{1}^{!} \mathcal{F}\right)$.

We will identify them freely in the following. If $\mathcal{F}$ and $\mathcal{G}$ are objects in the filtered derived categories, a homomorphism $u: c_{2}^{*} \mathcal{G} \rightarrow c_{1}^{!} \mathcal{F}$ in $D F_{\text {ctf }}(C)$ corresponds bijectively to a section of $c^{!} F_{0} R \mathcal{H}$ om $\left(\mathrm{pr}_{2}^{*} \mathcal{G}, \mathrm{pr}_{1}^{!} \mathcal{F}\right)$ by the isomorphism (1.6). If $c: C \rightarrow X \times Y$ is a closed immersion, the three notions above are further equivalent to the following:

(4) an element of $H_{C}^{0}\left(X \times Y, R \mathcal{H} o m\left(\operatorname{pr}_{2}^{*} \mathcal{G}, \operatorname{pr}_{1}^{!} \mathcal{F}\right)\right)$.

Recall that a canonical isomorphism

$$
R \mathcal{H} o m\left(\operatorname{pr}_{2}^{*} \mathcal{G}, \operatorname{pr}_{1}^{!} \mathcal{F}\right) \longrightarrow \mathcal{F} \otimes^{L} \mathbf{D} \mathcal{G}
$$

is defined in [1] (3.1.1).

A typical example of a cohomological correspondence is given as follows. Assume $X$ and $Y$ are smooth of dimension $d$ over $k$ and $c=\left(c_{1}, c_{2}\right): C \rightarrow X \times Y$ is a closed immersion. Let $\mathcal{F}$ and $\mathcal{G}$ be sheaves of free $\Lambda$-modules on $X$ and $Y$ respectively and assume $\mathcal{G}$ is smooth. Then, the canonical map $c^{*} \mathcal{H} o m\left(\operatorname{pr}_{2}^{*} \mathcal{G}, \operatorname{pr}_{1}^{*} \mathcal{F}\right) \rightarrow \mathcal{H o m}\left(c_{2}^{*} \mathcal{G}, c_{1}^{*} \mathcal{F}\right)$ 
is an isomorphism and we identify $\operatorname{Hom}\left(c_{2}^{*} \mathcal{G}, c_{1}^{*} \mathcal{F}\right)=\Gamma\left(C, c^{*} \mathcal{H o m}\left(\operatorname{pr}_{2}^{*} \mathcal{G}, \operatorname{pr}_{1}^{*} \mathcal{F}\right)\right)$. Since $\mathrm{pr}_{1}: X \times Y \rightarrow X$ is smooth, we have a canonical isomorphism $\operatorname{pr}_{1}^{*} \mathcal{F}(d)[2 d] \rightarrow \mathrm{pr}_{1}^{!} \mathcal{F}([6]$ 3.2.5) and we identify $R \mathcal{H} o m\left(\operatorname{pr}_{2}^{*} \mathcal{G}, \operatorname{pr}_{1}^{!} \mathcal{F}\right)=\mathcal{H} o m\left(\operatorname{pr}_{2}^{*} \mathcal{G}, \operatorname{pr}_{1}^{*} \mathcal{F}\right)(d)[2 d]$. Then the cycle class map $C H_{d}(C) \rightarrow H_{C}^{2 d}(X \times Y, \Lambda(d))$ induces a pairing

$$
\begin{aligned}
& C H_{d}(C) \otimes H o m\left(c_{2}^{*} \mathcal{G}, c_{1}^{*} \mathcal{F}\right) \longrightarrow H_{C}^{2 d}(X \times Y, \Lambda(d)) \otimes \Gamma\left(C, c^{*} \mathcal{H o m}\left(\operatorname{pr}_{2}^{*} \mathcal{G}, \operatorname{pr}_{1}^{*} \mathcal{F}\right)\right) \\
& \longrightarrow \quad H_{C}^{0}\left(X \times Y, R \mathcal{H} \text { om }\left(\operatorname{pr}_{2}^{*} \mathcal{G}, \mathrm{pr}_{1}^{!} \mathcal{F}\right)\right)
\end{aligned}
$$

In other words, the pair $(\Gamma, \gamma)$ of a cycle class $\Gamma \in C H_{d}(C)$ and a homomorphism $\gamma: c_{2}^{*} \mathcal{F} \rightarrow c_{1}^{*} \mathcal{F}$ defines a cohomological correspondence $u(\Gamma, \gamma)$.

We recall the definition of the push-forward of a cohomological correspondence. We consider a commutative diagram

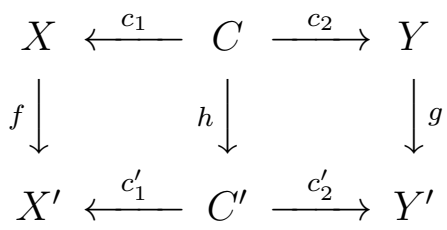

of schemes over $k$. A canonical isomorphism

$$
(f \times g)_{*} R \mathcal{H} o m\left(\operatorname{pr}_{2}^{*} \mathcal{G}, \operatorname{pr}_{1}^{!} \mathcal{F}\right) \rightarrow R \mathcal{H o m}\left(\operatorname{pr}_{2}^{*} g ! \mathcal{G}, \operatorname{pr}_{1}^{!} f_{*} \mathcal{F}\right)
$$

is defined in [11] (3.3.1), using the isomorphism (1.14). The diagram (1.16) defines a commutative diagram

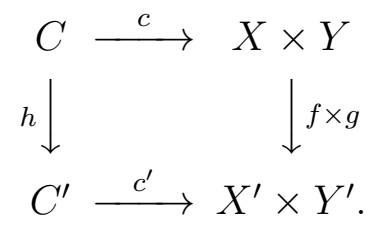

We assume the vertical arrows in (1.16) are proper. Let $u: c_{2}^{*} \mathcal{G} \rightarrow c_{1}^{!} \mathcal{F}$ be a cohomological correspondence. We identify $u$ with a section of $c^{!} R \mathcal{H}$ om $\left(\operatorname{pr}_{2}^{*} \mathcal{G}, \operatorname{pr}_{1}^{!} \mathcal{F}\right)$. Then, it defines a section $h_{*} u$ of $h_{*} c^{!} R \mathcal{H}$ om $\left(\operatorname{pr}_{2}^{*} \mathcal{G}, \operatorname{pr}_{1}^{!} \mathcal{F}\right)$. By the assumption that $f, g$ and $h$ are proper, the base change map (1.2) defines a map of functors $h_{*} c^{!}=h_{!} c^{!} \rightarrow c^{!}(f \times g) !=$ $c^{\prime !}(f \times g)_{*}$. This and the isomorphism (1.17) give maps

$$
\begin{aligned}
h_{*} c ! R \mathcal{H o m}\left(\operatorname{pr}_{2}^{*} \mathcal{G}, \operatorname{pr}_{1}^{!} \mathcal{F}\right) & \rightarrow c^{!}(f \times g)_{*} R \mathcal{H o m}\left(\mathrm{pr}_{2}^{*} \mathcal{G}, \mathrm{pr}_{1}^{!} \mathcal{F}\right) \\
& \rightarrow c^{\prime !} R \mathcal{H} \text { om }\left(\operatorname{pr}_{2}^{*} g_{!} \mathcal{G}, \operatorname{pr}_{1}^{!} f_{*} \mathcal{F}\right) .
\end{aligned}
$$

The push-forward $h_{*} u: c_{2}^{*} g_{*} \mathcal{G}=c_{2}^{*} g_{!} \mathcal{G} \rightarrow c_{1}^{\prime !} f_{*} \mathcal{F}$ is defined by the image of $h_{*} u$ by the composition. The push-forward $h_{*} u$ is equal to the composition of the maps

$$
c_{2}^{\prime *} g_{*} \mathcal{G} \stackrel{1.1}{\longrightarrow} h_{*} c_{2}^{*} \mathcal{G} \stackrel{h_{*}(u)}{\longrightarrow} h_{*} c_{1}^{!} \mathcal{F} \stackrel{1.2}{\longrightarrow} c_{1}^{\prime !} f_{*} \mathcal{F} .
$$

The formation of the push-forward is compatible with the composition. 
A variant $h_{!} u$ of $h_{*} u$ is defined if the map $c_{2}: C \rightarrow V$ in (1.16) is proper. In this case, we define $h_{!} u$ to be the composition

$$
c_{2}^{\prime *} g_{!} \mathcal{G} \stackrel{1.3}{\longrightarrow} h_{!} c_{2}^{*} \mathcal{G} \stackrel{h_{!}(u)}{\longrightarrow} h_{!} c_{1}^{!} \mathcal{F} \stackrel{1.2}{\longrightarrow} c_{1}^{\prime !} f_{!} \mathcal{F}
$$

The formation of the $h_{!} u$ is also compatible with the composition. If $f, g, h$ and $c_{2}$ are proper, we have $h_{*} u=h_{!} u$.

We study the restriction of a cohomological correspondence to an open subscheme. We consider a commutative diagram

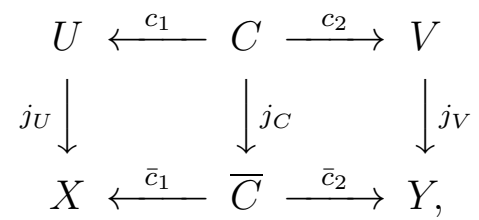

of schemes over $k$ where the vertical arrows are open immersions. Let $\mathcal{F}$ and $\mathcal{G}$ be objects of $D_{\text {ctf }}(X)$ and of $D_{\text {ctf }}(Y)$ respectively and $\bar{u}: \bar{c}_{2}^{*} \mathcal{G} \rightarrow \bar{c}_{1}^{!} \mathcal{F}$ be a cohomological correspondence on $\bar{C}$. Let $\mathcal{F}_{U}=j_{U}^{*} \mathcal{F}$ and $\mathcal{G}_{V}=j_{V}^{*} \mathcal{G}$ be the restrictions. We identify $j_{C}^{*} \bar{c}_{1}^{!} \mathcal{F}=j_{C}^{!} \bar{c}_{1}^{!} \mathcal{F}=c_{1}^{!} \mathcal{F}_{U}$ by the composition isomorphism. Then, the restriction $j_{C}^{*} \bar{u}$ on $C$ defines a cohomological correspondence $u: c_{2}^{*} \mathcal{G}_{V}=j_{C}^{*} \bar{c}_{2}^{*} \mathcal{G} \rightarrow j_{C}^{*} \bar{c}_{1}^{!} \mathcal{F}=c_{1}^{!} \mathcal{F}_{U}$.

Lemma 1.2.2 Let the notation be as above and let $j: U \times V \rightarrow X \times Y$ be the product $j_{U} \times j_{V}$. We put $\mathcal{H}=R \mathcal{H o m}\left(\mathrm{pr}_{2}^{*} \mathcal{G}_{V}, \mathrm{pr}_{1}^{!} \mathcal{F}_{U}\right)$ on $U \times V$ and $\overline{\mathcal{H}}=R \mathcal{H}$ om $\left(\mathrm{pr}_{2}^{*} \mathcal{G}, \mathrm{pr}_{1}^{!} \mathcal{F}\right)$ on $X \times Y$. We identify a cohomological correspondence $\bar{u}: \bar{c}_{2}^{*} \mathcal{G} \rightarrow \bar{c}_{1}^{!} \mathcal{F}$ with a section $\bar{u}$ of $\bar{c} \cdot \overline{\mathcal{H}}$ and the associated map $\bar{u}: \bar{c}_{!} \Lambda \rightarrow \overline{\mathcal{H}}$. We also identify the restriction $u=$ $j_{C}^{*} \bar{u}: c_{2}^{*} \mathcal{G}_{V} \rightarrow c_{1}^{!} \mathcal{F}_{U}$ with a section $u$ of $c^{!} \mathcal{H}$ and the associated map $u: c_{!} \Lambda \rightarrow \mathcal{H}$. Then, we have the following.

1. The section $u$ of $c^{!} \mathcal{H}$ is the image of the restriction of $\bar{u}$ by the composition isomorphism $j_{C}^{*} \bar{c}^{!} \overline{\mathcal{H}}=j_{C}^{!} \bar{c}^{!} \overline{\mathcal{H}} \rightarrow c^{!} j^{!} \overline{\mathcal{H}}=c^{!} j^{*} \overline{\mathcal{H}}=c^{!} \mathcal{H}$.

2. The square

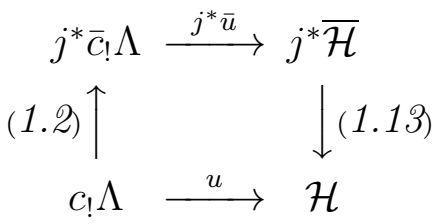

is commutative.

Proof. 1. The isomorphisms (1.13) form a commutative diagram

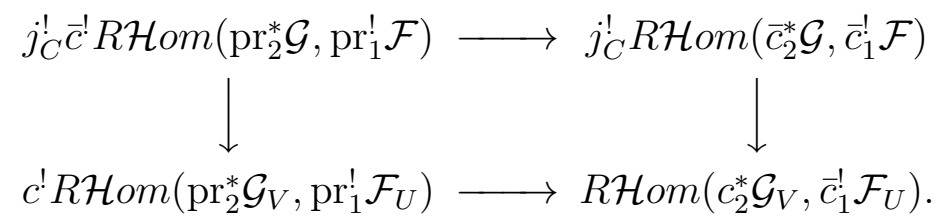

The assertion follows from this. 
2. We consider the diagram

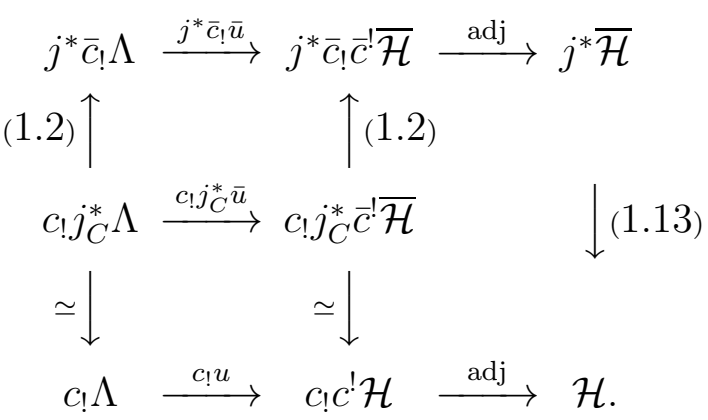

The upper left square is commutative by functoriality. The lower left square is commutative by 1 and the vertical arrows are isomorphisms. The right rectangle is commutative by the definition of the base change map (1.2). Since the square (1.20) is the outer square, the assertion is proved.

Lemma 1.2.3 Assume that the right square in the diagram (1.19) is cartesian. Let $\mathcal{F}$ and $\mathcal{G}$ be objects of $D_{\mathrm{ctf}}(U)$ and of $D_{\mathrm{ctf}}(V)$ respectively and $u: c_{2}^{*} \mathcal{G} \rightarrow c_{1}^{!} \mathcal{F}$ be a cohomological correspondence on $C$. Then, there exists a unique cohomological correspondence $\bar{u}: \bar{c}_{2}^{*} j_{V !} \mathcal{G} \rightarrow \bar{c}_{1}^{!} j_{U !} \mathcal{F}$ on $\bar{C}$ such that $j_{C}^{*} \bar{u}=u$.

Proof. Since the right square in (1.19) is assumed cartesian, we have $\bar{c}_{2}^{*} j_{V !}=j_{C !} c_{2}^{*}$. By adjunction, there exists a unique map $\bar{c}_{2}^{*} j_{V !} \mathcal{G}=j_{C !} c_{2}^{*} \mathcal{G} \rightarrow \bar{c}_{1}^{!} j_{U !} \mathcal{F}$ corresponding to $c_{2}^{*} \mathcal{G} \rightarrow j_{C}^{*} \bar{c}_{1}^{!} j_{U !} \mathcal{F}=c_{1}^{!} j_{C}^{*} j_{U !} \mathcal{F}$.

Corollary 1.2.4 1. Assume that the map $c_{2}: C \rightarrow V$ is proper and $C$ is dense in $\bar{C}$. Then the right square in the diagram (1.19) is cartesian.

2. Assume that the right square in the diagram (1.19) is cartesian. Let $\mathcal{F}$ and $\mathcal{G}$ be objects of $D_{\mathrm{ctf}}(U)$ and of $D_{\mathrm{ctf}}(V)$ respectively and $u: c_{2}^{*} \mathcal{G} \rightarrow c_{1}^{!} \mathcal{F}$ be a cohomological correspondence on $C$. Then, $\bar{u}=j_{C !} u: \bar{c}_{2}^{*} j_{V !} \mathcal{G} \rightarrow \bar{c}_{1}^{!} j_{U !} \mathcal{F}$ is the unique cohomological correspondence on $\bar{C}$ such that $j_{C}^{*} \bar{u}=u$.

Proof. 1. Since $C$ is closed and dense in $\bar{C} \times_{Y} V$, we have $C=\bar{C} \times_{Y} V$.

2. Clear from $j_{C}^{*} j_{C !} u=u$ and the uniqueness proved in Lemma 1.2.3.

We call $j_{C !} u: \bar{c}_{2}^{*} j_{V !} \mathcal{G} \rightarrow \bar{c}_{1}^{!} j_{U !} \mathcal{F}$ the zero-extension of $u$ (cf. [17] (2.3)). Similarly as the isomorphism (1.17), the isomorphism (1.14) induces a canonical isomorphism

$$
\left(1 \times j_{V}\right)_{*}\left(j_{U} \times 1\right) ! R \mathcal{H o m}\left(\mathrm{pr}_{2}^{*} \mathcal{G}, \mathrm{pr}_{1}^{!} \mathcal{F}\right) \rightarrow R \mathcal{H o m}\left(\mathrm{pr}_{2}^{*} j_{V !} \mathcal{G}, \mathrm{pr}_{1}^{!} j_{U !} \mathcal{F}\right)
$$

If the map $\bar{c}: \bar{C} \rightarrow X \times Y$ is a closed immersion and if $u: c_{2}^{*} \mathcal{G} \rightarrow c_{1}^{!} \mathcal{F}$ is identified with an element $u \in H_{C}^{0}\left(U \times V, R \mathcal{H} o m\left(\operatorname{pr}_{2}^{*} \mathcal{G}, \operatorname{pr}_{1}^{!} \mathcal{F}\right)\right)$, then the zero-extension $j_{C !} u$ is the inverse image of $u$ by the isomorphism

$$
\begin{aligned}
& H_{\bar{C}}^{0}\left(X \times Y, R \mathcal{H} \text { om }\left(\operatorname{pr}_{2}^{*} j_{V !} \mathcal{G}, \operatorname{pr}_{1}^{!} j_{U !} \mathcal{F}\right)\right) \\
\stackrel{\sim}{ } & H_{\bar{C}}^{0}\left(X \times Y,\left(1 \times j_{V}\right)_{*}\left(j_{U} \times 1\right)_{!} R \mathcal{H} \operatorname{om}\left(\operatorname{pr}_{2}^{*} \mathcal{G}, \operatorname{pr}_{1}^{!} \mathcal{F}\right)\right) \\
\rightarrow & H_{C}^{0}\left(X \times V,\left(j_{U} \times 1\right)_{!} R \mathcal{H} \operatorname{Hom}\left(\operatorname{pr}_{2}^{*} \mathcal{G}, \operatorname{pr}_{1}^{!} \mathcal{F}\right)\right)=H_{C}^{0}\left(U \times V, R \mathcal{H} o m\left(\operatorname{pr}_{2}^{*} \mathcal{G}, \operatorname{pr}_{1}^{!} \mathcal{F}\right)\right) .
\end{aligned}
$$


We define the pull-back of a cohomological correspondence. Let $f: X^{\prime} \rightarrow X$ and $g: Y^{\prime} \rightarrow Y$ be morphisms of smooth schemes over $k$. We assume $\operatorname{dim} X=\operatorname{dim} X^{\prime}$ and $\operatorname{dim} Y=\operatorname{dim} Y^{\prime}$. Let $\mathcal{F}$ and $\mathcal{G}$ be objects of $D_{\text {ctf }}(X)$ and of $D_{\text {ctf }}(Y)$ respectively. Then the canonical maps (1.9) and (1.13) induce a map

$$
\begin{aligned}
(f \times g)^{*} R \mathcal{H o m}\left(\operatorname{pr}_{2}^{*} \mathcal{G}, \operatorname{pr}_{1}^{!} \mathcal{F}\right) \rightarrow & (f \times g)^{!} R \mathcal{H} o m\left(\operatorname{pr}_{2}^{*} \mathcal{G}, \operatorname{pr}_{1}^{!} \mathcal{F}\right) \\
& \rightarrow R \mathcal{H} \text { om }\left(\operatorname{pr}_{2}^{\prime *} g^{*} \mathcal{G}, \operatorname{pr}_{1}^{\prime !} f^{!} \mathcal{F}\right) .
\end{aligned}
$$

With the isomorphism (1.14), the map (1.23) is identified with the composition

$$
(f \times g)^{*}(\mathcal{F} \otimes \mathbf{D} \mathcal{G}) \rightarrow f^{*} \mathcal{F} \otimes g^{*} \mathbf{D} \mathcal{G} \rightarrow f^{!} \mathcal{F} \otimes g^{!} \mathbf{D} \mathcal{G} \rightarrow f^{!} \mathcal{F} \otimes \mathbf{D} g^{*} \mathcal{G}
$$

where the middle arrow is defined by (1.9).

Let $c=\left(c_{1}, c_{2}\right): C \rightarrow X \times Y$ be a correspondence and $u: c_{2}^{*} \mathcal{G} \rightarrow c_{1}^{!} \mathcal{F}$ be a cohomological correspondence on $C$. We identify $u$ with a map $u: c_{!} \Lambda \rightarrow R \mathcal{H}$ om $\left(\operatorname{pr}_{2}^{*} \mathcal{G}, \operatorname{pr}_{1}^{!} \mathcal{F}\right)$ as above. We define a correspondence $c^{\prime}=\left(c_{1}^{\prime}, c_{2}^{\prime}\right): C^{\prime} \rightarrow X^{\prime} \times Y^{\prime}$ by the cartesian diagram

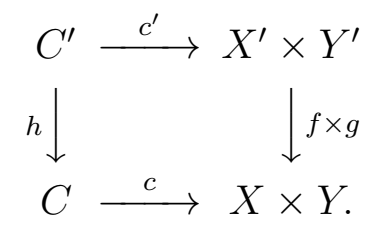

By the proper base change theorem, the base change map $(f \times g)^{*} c_{!} \Lambda \rightarrow c_{!}^{\prime} \Lambda$ is an isomorphism. Hence the map $u: c_{!} \Lambda \rightarrow R \mathcal{H}$ om $\left(\operatorname{pr}_{2}^{*} \mathcal{G}, \operatorname{pr}_{1}^{!} \mathcal{F}\right)$ induces a map

$$
c_{!}^{\prime} \Lambda \tilde{\leftarrow}(f \times g)^{*} c_{!} \Lambda \rightarrow(f \times g)^{*} R \mathcal{H o m}\left(\operatorname{pr}_{2}^{*} \mathcal{G}, \operatorname{pr}_{1}^{!} \mathcal{F}\right) \stackrel{1.23}{\longrightarrow} R \mathcal{H o m}\left(\operatorname{pr}_{2}^{*} g^{*} \mathcal{G}, \operatorname{pr}_{1}^{\prime} f^{!} \mathcal{F}\right) .
$$

The composition defines a cohomological correspondence $(f \times g)^{*} u: c_{2}^{\prime *} g^{*} \mathcal{G} \rightarrow c_{1}^{\prime !} f^{!} \mathcal{F}=$ $c_{1}^{\prime \prime} f^{*} \mathcal{F}$. We call $(f \times g)^{*} u$ the pull-back of $u$ by $f \times g$. If $c$ is a closed immersion, the correspondence $u: c_{2}^{*} \mathcal{G} \rightarrow c_{1}^{!} \mathcal{F}$ is identified with a cohomology class $u \in H_{C}^{0}(X \times$ $\left.Y, R \mathcal{H o m}\left(\operatorname{pr}_{2}^{*} \mathcal{G}, \operatorname{pr}_{1}^{\prime} \mathcal{F}\right)\right)$ and the pull-back $(f \times g)^{*} u$ is identified with the pull-back $(f \times g)^{*} u \in H_{C^{\prime}}^{0}\left(X^{\prime} \times Y^{\prime}, R \mathcal{H}\right.$ om $\left.\left(\operatorname{pr}_{2}^{\prime *} g^{*} \mathcal{G}, \operatorname{pr}_{1}^{\prime} f^{!} \mathcal{F}\right)\right)$.

For a cohomological correspondence $u(\Gamma, \gamma)$, its pull-back is computed by using the intersection product.

Lemma 1.2.5 Let the notation be as above. The diagram

$$
\begin{aligned}
& C H_{d}(C) \otimes H o m\left(c_{2}^{*} \mathcal{G}, c_{1}^{*} \mathcal{F}\right) \stackrel{1.15}{\longrightarrow} \quad H_{C}^{0}\left(X \times Y, R \mathcal{H o m}\left(\operatorname{pr}_{2}^{*} \mathcal{G}, \operatorname{pr}_{1}^{!} \mathcal{F}\right)\right) \\
& (f \times g)^{!} \otimes h^{*} \downarrow \quad \downarrow(f \times g)^{*} \\
& C H_{d}\left(C^{\prime}\right) \otimes H o m\left(c_{2}^{\prime *} g^{*} \mathcal{G}, c_{1}^{\prime *} f^{*} \mathcal{F}\right) \stackrel{1.15}{\longrightarrow} H_{C^{\prime}}^{0}\left(X^{\prime} \times Y^{\prime}, R \mathcal{H o m}\left(\operatorname{pr}_{2}^{*} g^{*} \mathcal{G}, \operatorname{pr}_{1}^{!} f^{*} \mathcal{F}\right)\right)
\end{aligned}
$$

is commutative.

Proof. Clear from the commutative diagram (1.11). 


\subsection{Complement on the Verdier pairing.}

We briefly recall the definition of the pairing [11] (4.2.5). We consider a cartesian diagram

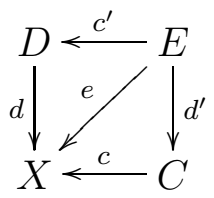

of schemes over $k$. Let $\mathcal{P}$ and $\mathcal{Q}$ be objects of $D_{\text {ctf }}(X)$ and let $\mathcal{P} \otimes \mathcal{Q} \rightarrow \mathcal{K}_{X}$ be a morphism. Then, the map (4.2.1) in 11] and the map $\mathcal{P} \otimes \mathcal{Q} \rightarrow \mathcal{K}_{X}$ induce a map $c^{!} \mathcal{P} \otimes d^{!} \mathcal{Q} \rightarrow e^{!} \mathcal{K}_{X}=\mathcal{K}_{E}$ and hence a pairing

$$
\langle,\rangle: H^{0}\left(C, c^{!} \mathcal{P}\right) \otimes H^{0}\left(D, d^{!} \mathcal{Q}\right) \rightarrow H^{0}\left(E, e^{!} \mathcal{K}_{X}\right)=H^{0}\left(E, \mathcal{K}_{E}\right)
$$

If the maps $c: C \rightarrow X$ and $d: D \rightarrow X$ are closed immersions, the pairing (1.27) gives a pairing $\langle\rangle:, H_{C}^{0}(X, \mathcal{P}) \otimes H_{D}^{0}(X, \mathcal{Q}) \rightarrow H_{E}^{0}\left(X, \mathcal{K}_{X}\right)=H^{0}\left(E, \mathcal{K}_{E}\right)$.

Lemma 1.3.1 Let the notation be as above. Then, we have a commutative diagram

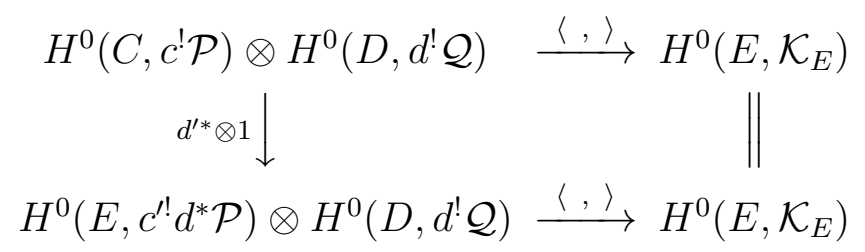

where the lower horizontal arrow is the pairing (1.27) for $E \stackrel{c^{\prime}}{\rightarrow} D \stackrel{\text { id }}{\leftarrow} D$ and the map $d^{*} \mathcal{P} \otimes d^{!} \mathcal{Q} \rightarrow d^{!} \mathcal{K}_{X}=\mathcal{K}_{D}(1.7)$ induced by $\mathcal{P} \otimes \mathcal{Q} \rightarrow \mathcal{K}_{X}$

The pairing $\langle$,$\rangle is compatible with the proper push-forward in the following sense.$

\section{Lemma 1.3.2 Let}

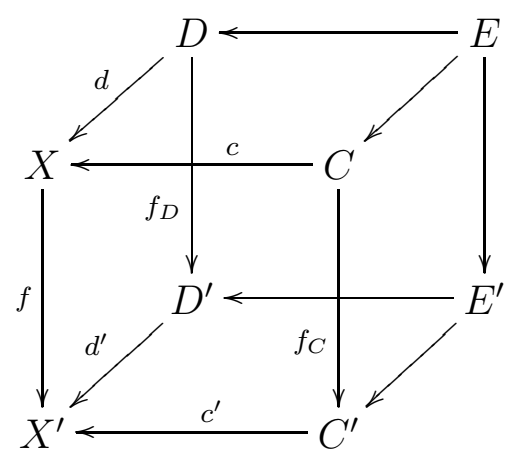

be a commutative cube of schemes over $k$. We assume that the horizontal faces are cartesian and that vertical arrows are proper. Let $\mathcal{P}$ and $\mathcal{Q}$ be objects of $D_{\text {ctf }}(X)$ and let $\mathcal{P} \otimes \mathcal{Q} \rightarrow \mathcal{K}_{X}$ be a morphism. 
Then, the pairings $\langle$,$\rangle for \mathcal{P} \otimes \mathcal{Q} \rightarrow \mathcal{K}_{X}$ and for $f_{*} \mathcal{P} \otimes f_{*} \mathcal{Q} \rightarrow f_{*} \mathcal{K}_{X} \rightarrow \mathcal{K}_{X^{\prime}}$ form a commutative diagram

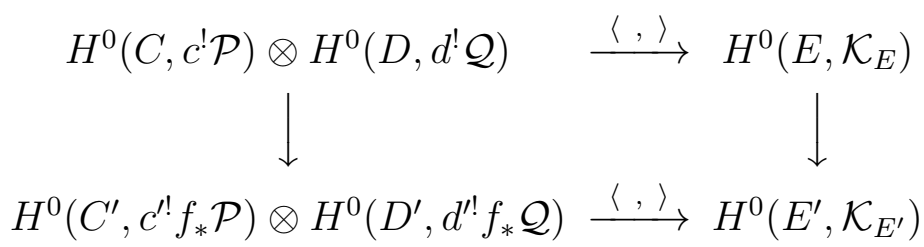

where the left vertical maps are induced by the base change maps $f_{C *} c^{!} \rightarrow c^{\prime !} f_{*}$ and $f_{D *} d^{!} \rightarrow d^{\prime !} f_{*}(1.2)$.

Proof. It follows from the commutativity of the squares (A) and (B) in the diagram [1] (4.4.2).

Applying the pairing (1.27) to cohomological correspondences, we obtain the Verdier pairing. Let $X$ and $Y$ be schemes over $k$ and

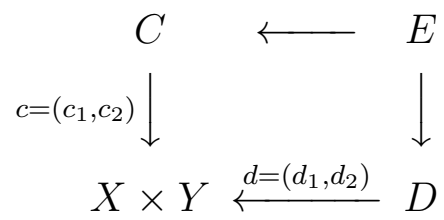

be a cartesian diagram of schemes over $k$. Let $\mathcal{F}$ and $\mathcal{G}$ be objects of $D_{\text {ctf }}(X)$ and of $D_{\text {ctf }}(Y)$ respectively. We put $\mathcal{H}=R \mathcal{H} o m\left(\operatorname{pr}_{2}^{*} \mathcal{G}, \operatorname{pr}_{1}^{!} \mathcal{F}\right)$ and $\mathcal{H}^{*}=R \mathcal{H o m}\left(\operatorname{pr}_{1}^{*} \mathcal{F}, \operatorname{pr}_{2}^{!} \mathcal{G}\right)$ on $X \times Y$. A canonical map

$$
\mathcal{H} \otimes \mathcal{H}^{*} \rightarrow \mathcal{K}_{X \times Y}
$$

is defined in [1] (4.1.4), using the isomorphism (1.14). If $X$ and $Y$ are smooth over $k$ of dimension $d$ and if $\mathcal{F}$ and $\mathcal{G}$ are smooth sheaves of free $\Lambda$-modules, we have $\mathcal{H}=\mathcal{H o m}\left(\operatorname{pr}_{2}^{*} \mathcal{G}, \operatorname{pr}_{1}^{*} \mathcal{F}\right)(d)[2 d]$ and $\mathcal{H}^{*}=\mathcal{H} o m\left(\operatorname{pr}_{1}^{*} \mathcal{F}, \operatorname{pr}_{2}^{*} \mathcal{G}\right)(d)[2 d]$ and the map (1.30) is induced by the pairing $\mathcal{H} o m\left(\operatorname{pr}_{2}^{*} \mathcal{G}, \operatorname{pr}_{1}^{*} \mathcal{F}\right) \otimes \mathcal{H} o m\left(\operatorname{pr}_{1}^{*} \mathcal{F}, \operatorname{pr}_{2}^{*} \mathcal{G}\right) \rightarrow \Lambda: a \otimes b \mapsto \operatorname{Tr}(b \circ a)$. The pairing (1.27) defines the Verdier pairing

$$
\langle,\rangle: \operatorname{Hom}\left(c_{2}^{*} \mathcal{G}, c_{1}^{!} \mathcal{F}\right) \otimes \operatorname{Hom}\left(d_{2}^{*} \mathcal{F}, d_{1}^{!} \mathcal{G}\right) \rightarrow H^{0}\left(E, \mathcal{K}_{E}\right)
$$

as in 11 (4.2.5).

The following result will be useful in computing the Verdier pairing of the extension by zero of a correspondence.

Proposition 1.3.3 Let $X$ and $Y$ be schemes over $k$ and $j_{U}: U \rightarrow X$ and $j_{V}: V \rightarrow Y$ be open immersions. Let $f:(X \times Y)^{\prime} \rightarrow X \times Y$ be a proper morphism over $k$ that is an isomorphism on $(X \times V) \cup(U \times Y)$; we identify $(X \times V) \cup(U \times Y)$ as an open 
subscheme of $(X \times Y)^{\prime}$ by the restriction of $f$. We consider a commutative diagram

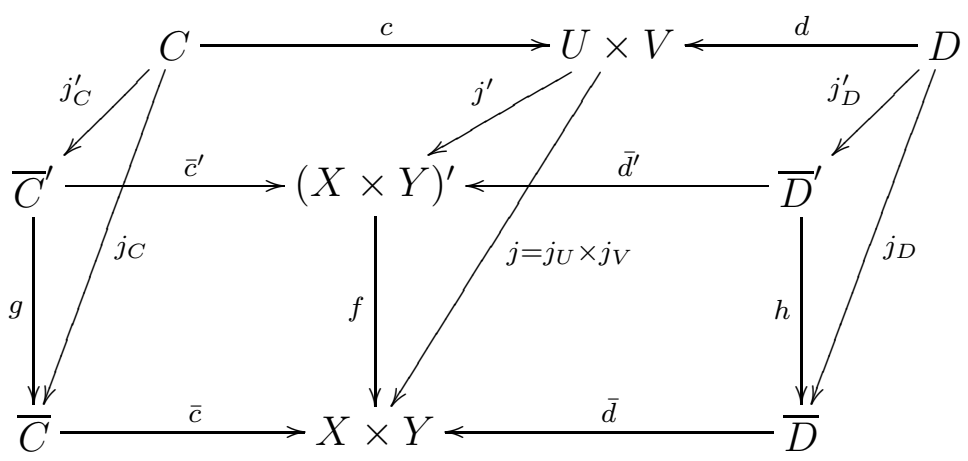

of schemes over $k$. We assume that the vertial arrows $f, g, h$ and the horizontal arrows $c, \bar{c}, \bar{c}^{\prime}, d, \bar{d}, \bar{d}^{\prime}$ are proper and that the four slant parallelograms are cartesian, so that the six slant arrows are open immersions. We also assume $C=\bar{C} \times_{X \times Y}(X \times V)$ and $D=\bar{D} \times_{X \times Y}(U \times Y)$.

Let $\mathcal{F}$ be an object of $D_{\mathrm{ctf}}(U)$ and $\mathcal{G}$ be an object of $D_{\mathrm{ctf}}(V)$ respectively. $W e$ put $\mathcal{H}=R \mathcal{H o m}\left(\mathrm{pr}_{2}^{*} \mathcal{G}, \mathrm{pr}_{1}^{!} \mathcal{F}\right)$ and $\mathcal{H}^{*}=R \mathcal{H} o m\left(\operatorname{pr}_{1}^{*} \mathcal{F}, \operatorname{pr}_{2}^{!} \mathcal{G}\right)$ on $U \times V$ and $\overline{\mathcal{H}}=$ $R \mathcal{H o m}\left(\mathrm{pr}_{2}^{*} j_{V !} \mathcal{G}, \mathrm{pr}_{1}^{!} j_{U !} \mathcal{F}\right)$ and $\overline{\mathcal{H}}^{*}=R \mathcal{H}$ om $\left(\mathrm{pr}_{1}^{*} j_{U !} \mathcal{F}, \mathrm{pr}_{2}^{!} j_{V !} \mathcal{G}\right)$ on $X \times Y$. Let $\overline{\mathcal{H}}^{\prime}$ and $\overline{\mathcal{H}}^{* \prime}$ be objects of $D_{\text {ctf }}\left((X \times Y)^{\prime}\right)$. Let $u: c_{!} \Lambda \rightarrow \mathcal{H}$ and $v: d_{!} \Lambda \rightarrow \mathcal{H}^{*}$ be cohomological correspondences.

1. For morphisms $a:\left.\left.\overline{\mathcal{H}}\right|_{U \times Y} \rightarrow \overline{\mathcal{H}}^{\prime}\right|_{U \times Y}$ and $b:\left.\left.\overline{\mathcal{H}}^{*}\right|_{X \times V} \rightarrow \overline{\mathcal{H}}^{* \prime}\right|_{X \times V}$, there exist unique morphisms $\bar{a}: \overline{\mathcal{H}} \rightarrow f_{*} \overline{\mathcal{H}}^{\prime}$ and $\bar{b}: \overline{\mathcal{H}}^{*} \rightarrow f_{*} \overline{\mathcal{H}}^{* \prime}$ extending a and $b$ respectively.

2. Let $\bar{u}^{\prime}: \bar{c}_{1}^{\prime} \Lambda \rightarrow \overline{\mathcal{H}}^{\prime}$ and $\bar{v}^{\prime}: \bar{d}_{!}^{\prime} \Lambda \rightarrow \overline{\mathcal{H}}^{\prime *}$ be morphisms and $\bar{a}: \overline{\mathcal{H}} \rightarrow f_{*} \overline{\mathcal{H}}^{\prime}$ and $\bar{b}: \overline{\mathcal{H}}^{*} \rightarrow f_{*} \overline{\mathcal{H}}^{* \prime}$ be isomorphisms. We identify the functor $j^{*} f_{*}$ with $j^{* *}$ by the isomorphism $j^{* *} \rightarrow j^{*} f_{*}(1.2)$. Suppose the squares

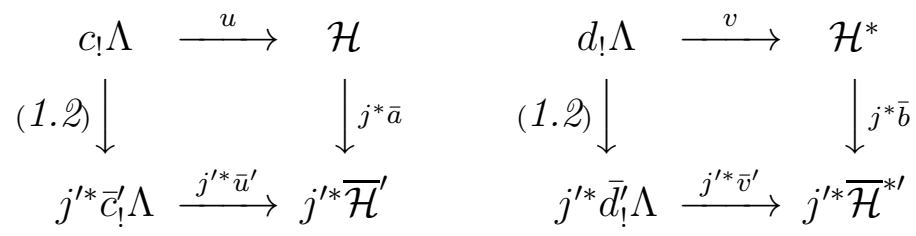

are commutative. Then, for the zero-extensions $j_{C !} u$ and $j_{D !} v$, the squares

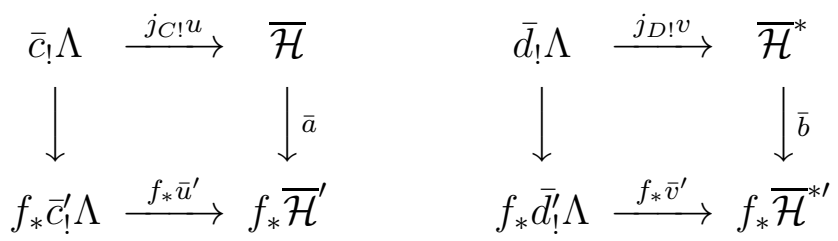

are commutative, where the left vertical arrows are induced by the canonical maps $\Lambda \rightarrow g_{*} \Lambda$ and $\Lambda \rightarrow h_{*} \Lambda$.

3. We keep the assumpions in 2. Further, let $\overline{\mathcal{H}}^{\prime} \otimes \overline{\mathcal{H}}^{* \prime} \rightarrow \mathcal{K}_{(X \times Y)^{\prime}}$ be a map making 
the square

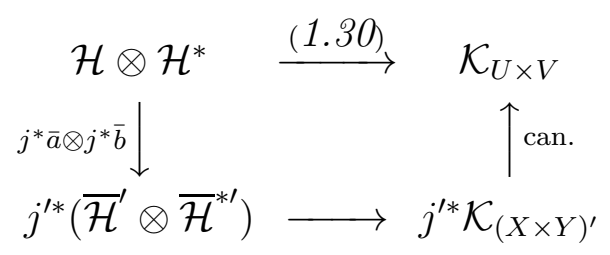

commutative. Then, we have

$$
\left\langle j_{C !} u, j_{D !} v\right\rangle=f_{*}\left\langle\bar{u}^{\prime}, \bar{v}^{\prime}\right\rangle
$$

in $H^{0}\left(E, \mathcal{K}_{E}\right)$.

Proof. 1. Since $\overline{\mathcal{H}}=\left.\left(j_{U} \times 1\right) ! \mathcal{H}\right|_{U \times Y}$ and $\overline{\mathcal{H}}^{*}=\left.\left(1 \times j_{V}\right) ! \mathcal{H}^{*}\right|_{X \times V}$, the assertion follows.

2. We show the assertion for $u$. The proof for $v$ is similar and omitted. We claim that we obtain (1.33) by applying $j^{*}$ to (1.34). This is clear for the bottom line and the right column. For the top line, we have $j_{C}^{*} j_{C !} u=u$ by Corollary 1.2.4 2. We show it for the left column. It suffices to show the diagram

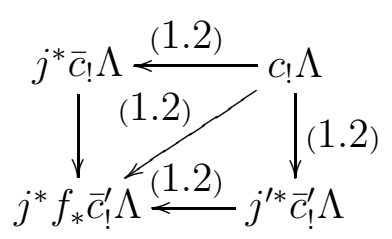

is commutative. The lower right triangle is commutative by the transitivity (1.4) of the base change maps (1.2) for the base change of $\bar{C} \rightarrow(X \times Y)^{\prime} \rightarrow X \times Y$ by $j: U \times V \rightarrow X \times Y$. Since the base change map (1.2) is the inverse of (1.1), the upper left triangle is the adjoint of the transitivity for the canonical maps $\bar{c}_{!} \Lambda \rightarrow f_{*} \bar{c}_{!}^{\prime} \Lambda \rightarrow j_{*} c_{!} \Lambda$ and is commutative. Hence, the left column in (1.34) also induces that in (1.33).

Since $\bar{a}$ is an isomorphism, there exists a unique map $\bar{u}: \bar{c}_{!} \Lambda \rightarrow \overline{\mathcal{H}}$ that makes the square (1.34) commutative. Since $u$ is the unique map that makes the square (1.33) commutative, we have $j_{C}^{*} \bar{u}=u$. Thus we obtain $\bar{u}=j_{C !} u$ by Lemma 1.2.3.

3. We defined the push forward $f_{*} \bar{u}^{\prime}: \Lambda \rightarrow g_{*} \bar{c}^{\prime} \overline{\mathcal{H}}^{\prime} \rightarrow \bar{c}^{!} f_{*} \overline{\mathcal{H}}^{\prime}$ and proved $f_{*}\left\langle\bar{u}^{\prime}, \bar{v}^{\prime}\right\rangle=$ $\left\langle f_{*} \bar{u}^{\prime}, f_{*} \bar{v}^{\prime}\right\rangle$ in Lemma 1.3.2. The push forward $f_{*} \bar{u}^{\prime}$ is the adjoint of the composition $\bar{c} ! \Lambda \rightarrow f_{*} \bar{c}_{!}^{\prime} \Lambda \stackrel{f_{*} \bar{u}^{\prime}}{\rightarrow} f_{*} \overline{\mathcal{H}}^{\prime}$ and is equal to $\bar{a} \circ j_{C !} u$ by 2 . Hence, we have $\left\langle f_{*} \bar{u}^{\prime}, f_{*} \bar{v}^{\prime}\right\rangle=$ $\left\langle\bar{a} \circ j_{C !} u, \bar{b} \circ j_{D !} v\right\rangle$. Thus, it suffices to show $\left\langle j_{C !} u, j_{D !} v\right\rangle=\left\langle\bar{a} \circ j_{C !} u, \bar{b} \circ j_{D !} v\right\rangle$.

Since $\overline{\mathcal{H}} \otimes \overline{\mathcal{H}}^{*}=j_{!}\left(\mathcal{H} \otimes \mathcal{H}^{*}\right)$, we obtain a commutative diagram

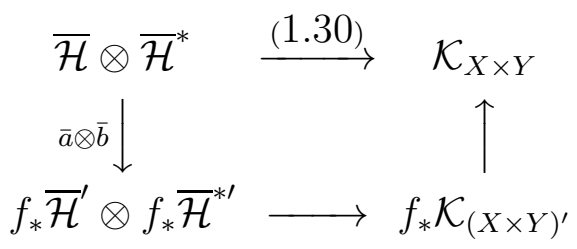

where the lower horizontal arrow is induced by $\overline{\mathcal{H}}^{\prime} \otimes \overline{\mathcal{H}}^{* \prime} \rightarrow \mathcal{K}_{(X \times Y)^{\prime}}$. Thus, we have $\left\langle j_{C !} u, j_{D !} v\right\rangle=\left\langle\bar{a} \circ j_{C !} u, \bar{b} \circ j_{D !} v\right\rangle$ as required. 


\section{Characteristic class of an $\ell$-adic étale sheaf.}

\subsection{Characteristic class of a cohomological correpondence.}

Let $X$ be a scheme over $k$ and $\delta: X=\Delta_{X} \rightarrow X \times X$ be the diagonal map. Let $\mathcal{F}$ be an object of $D_{\text {ctf }}(X)$ and let $1=u(X, 1)$ be the cohomological correspondence defined by the identity of $\mathcal{F}$ on the diagonal $X$.

Definition 2.1.1 Let $c=\left(c_{1}, c_{2}\right): C \rightarrow X \times X$ be a closed immersion and $u: c_{2}^{*} \mathcal{F} \rightarrow$ $c_{1}^{!} \mathcal{F}$ be a cohomological correspondence on $C$. Then, we call the cohomology class $\langle u, 1\rangle \in H_{C \cap X}^{0}\left(X, \mathcal{K}_{X}\right)$ defined in (1.31) the characteristic class and write $C(\mathcal{F}, C, u)$.

If $C=X$ is the diagonal and $u: \mathcal{F} \rightarrow \mathcal{F}$ is an endomorphism, we drop $C$ from the notation and we write $C(\mathcal{F}, u) \in H^{0}\left(X, \mathcal{K}_{X}\right)$. Further, if $u$ is the identity, we simply write $C(\mathcal{F})=\langle 1,1\rangle$ and call it the characteristic class of $\mathcal{F}$.

Example 2.1.2 ([13] Exercise I.32) If $X=C=\operatorname{Spec} k$, we have $C(\mathcal{F}, u)=\operatorname{Tr} u \in$ $\Lambda$. In particular, if $u=1$, we have $C(\mathcal{F})=\chi(\mathcal{F})$.

Lemma 2.1.3 Let $X$ be a scheme over $k$ and $c=\left(c_{1}, c_{2}\right): C \rightarrow X \times X$ be a closed immersion. Let $\left(\mathcal{F}, F_{\bullet}\right)$ be an object of the filtered derived category $D F_{\text {ctf }}(X)$ and $u: c_{2}^{*} \mathcal{F} \rightarrow c_{1}^{!} \mathcal{F}$ be a morphism in the filtered derived category $D F_{\text {ctf }}(C)$. Then, we have

$$
C(\mathcal{F}, C, u)=\sum_{q} C\left(\operatorname{Gr}_{q}^{F} \mathcal{F}, C, \operatorname{Gr}_{q}^{F} u\right)
$$

in $H^{0}\left(X, \mathcal{K}_{X}\right)$.

Proof. It is a special case of the equality [11] (4.13.1). For later use, we briefly skecth the proof. By the isomorphism (1.6), the homomorphism $u \in \operatorname{Hom}_{D F_{\text {ctf }}(C)}\left(c_{2}^{*} \mathcal{F}, c_{1}^{!} \mathcal{F}\right)$ defines a section $u$ of $c^{!} F_{0} R \mathcal{H} o m\left(\operatorname{pr}_{2}^{*} \mathcal{F}, \operatorname{pr}_{1}^{!} \mathcal{F}\right)$ on $C$. Similarly the identity defines a section 1 of $\delta^{!} F_{0} R \mathcal{H}$ om $\left(\mathrm{pr}_{1}^{*} \mathcal{F}, \mathrm{pr}_{2}^{!} \mathcal{F}\right)$ on the diagonal $X$. We have a canonical isomorphism $G r_{0}^{F} R \mathcal{H} o m\left(\operatorname{pr}_{2}^{*} \mathcal{F}, \operatorname{pr}_{1}^{!} \mathcal{F}\right) \rightarrow \bigoplus_{q} R \mathcal{H}$ om $\left(\operatorname{pr}_{2}^{*} G r_{q}^{F} \mathcal{F}, \operatorname{pr}_{1}^{!} G r_{q}^{F} \mathcal{F}\right)$ and similarly for $G r_{0}^{F} R \mathcal{H}$ om $\left(\operatorname{pr}_{1}^{*} \mathcal{F}, \mathrm{pr}_{2}^{!} \mathcal{F}\right)$. Since the restriction of the canonical pairing (1.27) on $F_{0} R \mathcal{H}$ om $\left(\operatorname{pr}_{2}^{*} \mathcal{F}, \operatorname{pr}_{1}^{!} \mathcal{F}\right) \otimes F_{0} R \mathcal{H} o m\left(\operatorname{pr}_{1}^{*} \mathcal{F}, \operatorname{pr}_{2}^{!} \mathcal{F}\right)$ is induced by the sum of the canonical pairings $R \mathcal{H}$ om $\left(\operatorname{pr}_{2}^{*} G r_{q}^{F} \mathcal{F}, \operatorname{pr}_{1}^{!} G r_{q}^{F} \mathcal{F}\right) \otimes R \mathcal{H} o m\left(\operatorname{pr}_{1}^{*} G r_{q}^{F} \mathcal{F}, \operatorname{pr}_{2}^{!} G r_{q}^{F} \mathcal{F}\right) \rightarrow \mathcal{K}_{X \times X}$, the assertion follows.

Remark 2.1.4 ([9]) For a distinguished triangle $\rightarrow \mathcal{F} \stackrel{u}{\rightarrow} \mathcal{G} \stackrel{v}{\rightarrow} \mathcal{H} \stackrel{w}{\rightarrow}$ of perfect complexes of $\Lambda$-modules and endomorphisms $f, g, h$ of $\mathcal{F}, \mathcal{G}, \mathcal{H}$ compatible with $u, v, w$, there is a counterexample to the equality $\operatorname{Tr}(g)=\operatorname{Tr}(f)+\operatorname{Tr}(h)$.

Corollary 2.1.5 Let $X$ be a scheme over $k$ and $(\mathcal{F}, F)$ be an object of $D F_{\mathrm{ctf}}(X)$. Then, we have

$$
C(\mathcal{F})=\sum_{q} C\left(G r_{q}^{F} \mathcal{F}\right)
$$

in $H^{0}\left(X, \mathcal{K}_{X}\right)$. 
Proposition 2.1.6 (11] Théorème 4.4, Corollaire 4.8) Let $f: X \rightarrow Y$ be a proper morphism of schemes over $k$ and we consider a commutative diagram

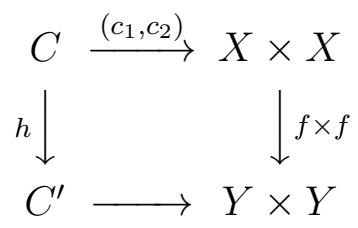

of schemes over $k$ where the vertical arrows are proper and the horizontal arrows are closed immersions. Let $u: c_{2}^{*} \mathcal{F} \rightarrow c_{1}^{\prime} \mathcal{F}$ be a cohomological correspondence. Then we have

$$
C\left(f_{*} \mathcal{F}, C^{\prime}, h_{*} u\right)=f_{*} C(\mathcal{F}, C, u) .
$$

In particular, if $X$ is proper over $k$, we have

$$
\operatorname{Tr}\left(u^{*}: H^{*}\left(X_{\bar{k}}, \mathcal{F}\right)\right)=\operatorname{Tr} C(\mathcal{F}, C, u),
$$

where $\bar{k}$ is a separable closure of $k$.

By devissage using Lemma 2.1.3 and Proposition 2.1.6, the computation of a characteric class is reduced to the computation of $C(j ! \mathcal{F})$ where $j: U \rightarrow X$ is an open immersion, $U$ is smooth and the cohomology sheaves $\mathcal{H}^{q} \mathcal{F}$ are locally constant on $U$. We will later compute the characteristic class $C(j ! \mathcal{F})$ or rather the difference $C(j ! \mathcal{F})-\operatorname{rank} \mathcal{F} \cdot C(j ! \Lambda)$ in terms of the ramification of $\mathcal{F}$ along the boundary $X \backslash U$.

We give an equivalent description of the characteristic class. The canonical isomorphism (1.14)

$$
\mathcal{H}=R \mathcal{H} o m\left(\operatorname{pr}_{2}^{*} \mathcal{F}, \operatorname{pr}_{1}^{!} \mathcal{F}\right) \longrightarrow \mathcal{F} \otimes^{L} \mathbf{D} \mathcal{F}
$$

induces an isomorphism

$$
\delta^{*} \mathcal{H} \longrightarrow \mathcal{F} \otimes^{L} \mathbf{D} \mathcal{F}
$$

Thus the evaluation map

$$
\mathcal{F} \otimes{ }^{L} \mathbf{D} \mathcal{F} \longrightarrow \mathcal{K}_{X}
$$

[11] (2.2.2) induces a map

$$
\delta^{*} \mathcal{H} \longrightarrow \mathcal{K}_{X}
$$

If $X$ is smooth of dimension $d$ and if $\mathcal{F}$ is a smooth sheaf of free $\Lambda$-modules, the map (2.4) is equal to the composition of

$$
\delta^{*} \mathcal{H}=\delta^{*} \mathcal{H o m}\left(\operatorname{pr}_{2}^{*} \mathcal{F}, \operatorname{pr}_{1}^{*} \mathcal{F}\right)(d)[2 d]=\mathcal{E} n d(\mathcal{F})(d)[2 d] \stackrel{\operatorname{Tr}}{\longrightarrow} \Lambda(d)[2 d] \rightarrow \mathcal{K}_{X} .
$$

If we put $\mathcal{H}^{*}=R \mathcal{H}$ om $\left(\mathrm{pr}_{1}^{*} \mathcal{F}, \operatorname{pr}_{2}^{!} \mathcal{F}\right)$, the map (2.4) is the dual of the map $\Lambda_{X} \rightarrow \delta^{!} \mathcal{H}^{*}$ corresponding to $1=u(X, 1)$. 
Proposition 2.1.7 Let $c: C \rightarrow X \times X$ be a closed immersion and $u: c_{!} \Lambda_{C} \rightarrow \mathcal{H}=$ $R \mathcal{H}$ om $\left(\operatorname{pr}_{2}^{*} \mathcal{F}, \operatorname{pr}_{1}^{!} \mathcal{F}\right)$ be a cohomological correspondence. Let $i: C \cap X \rightarrow X$ denote the immersion. Then the characteristic class $C(\mathcal{F}, C, u) \in H_{C \cap X}^{0}\left(X, \mathcal{K}_{X}\right)$ is equal to the cohomology class of the composition

$$
i_{!} \Lambda_{C \cap X}=\delta^{*} c_{!} \Lambda_{C} \stackrel{\delta^{*}(u)}{\longrightarrow} \delta^{*} \mathcal{H} \stackrel{2.4}{\longrightarrow} \mathcal{K}_{X}
$$

Proof. Let 1: $\Lambda_{X} \rightarrow \delta ! \mathcal{H}^{*}$ be the cohomological correspondence defined by the identity of $\mathcal{F}$. Then the evaluation map (2.4) is equal to the composition of

$$
\delta^{*} \mathcal{H} \stackrel{\operatorname{id} \otimes 1}{\longrightarrow} \delta^{*} \mathcal{H} \otimes \delta^{!} \mathcal{H}^{*} \stackrel{1.7}{\longrightarrow} \delta^{!}\left(\mathcal{H} \otimes \mathcal{H}^{*}\right) \longrightarrow \delta^{!} \mathcal{K}_{X \times X}=\mathcal{K}_{X}
$$

where the last arrow is induced by the pairing (1.30). Thus the assertion follows from Lemma 1.3.1

We define a refinement of the characteristic class for zero-extensions. Let $X$ be a scheme over $k$ and $j_{U}: U \rightarrow X$ be an open immersion over $k$. Let $\delta: X \rightarrow X \times X$ and $\delta_{U}: U \rightarrow U \times U$ be the diagonal maps. Let $\mathcal{F}$ be an object of $D_{\text {ctf }}(U)$. We put $\mathcal{H}=R \mathcal{H}$ om $\left(\operatorname{pr}_{2}^{*} \mathcal{F}, \operatorname{pr}_{1}^{!} \mathcal{F}\right)$ on $U \times U$ and $\overline{\mathcal{H}}=R \mathcal{H}$ om $\left(\operatorname{pr}_{2}^{*} j_{U !} \mathcal{F}, \operatorname{pr}_{1}^{!} j_{U !} \mathcal{F}\right)$ on $X \times X$. Since $\overline{\mathcal{H}}=\left(j_{U} \times 1\right)_{!}\left(1 \times j_{U}\right)_{*} \mathcal{H}$, we have a canonical isomorphism $j_{U !} \delta_{U}^{*} \mathcal{H} \rightarrow \delta^{*} \overline{\mathcal{H}}$. Thus the evaluation map $e: \delta_{U}^{*} \mathcal{H} \rightarrow \mathcal{K}_{U}(\underline{2.4})$ on $U$ induces its zero-extension $j_{U !} e: \delta^{*} \overline{\mathcal{H}} \rightarrow j_{U !} \mathcal{K}_{U}$.

Definition 2.1.8 Let $X$ be a scheme over $k$ and $j_{U}: U \rightarrow X$ be an open immersion over $k$. Let $\bar{C}$ be a closed subscheme of $X \times X$ and we consider the cartesian diagram

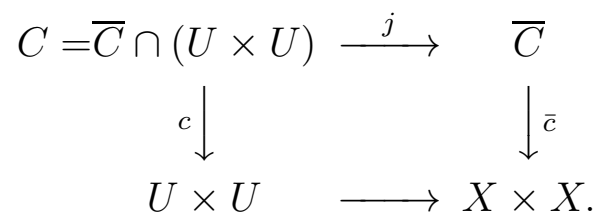

We assume $C=\bar{C} \cap(X \times U)$. Let $\bar{i}: \bar{C} \cap X \rightarrow X$ denotes the closed immersion. Let $\mathcal{F}$ be an object of $D_{\text {ctf }}(U)$. Let $u: c_{1} \Lambda \rightarrow \mathcal{H}$ be a cohomological correspondence and let $j_{!} u: \bar{c}_{!} \Lambda \rightarrow \overline{\mathcal{H}}$ be the zero-extension. Then, we define the refinement $C_{!}\left(j_{U !} \mathcal{F}, \bar{C}, j_{!} u\right) \in$ $H_{\bar{C} \cap X}^{0}\left(X, j_{U !} \mathcal{K}_{U}\right)$ of the characteristic class to be the class of the composition

$$
\bar{i}_{!} \Lambda_{\bar{C} \cap X}=\delta^{*} \bar{c}_{!} \Lambda_{C} \stackrel{\delta^{*}(j ! u)}{\longrightarrow} \delta^{*} \overline{\mathcal{H}} \stackrel{j_{U !} e}{\longrightarrow} j_{U !} \mathcal{K}_{U} .
$$

If $\bar{C}$ is the diagonal and $u: \mathcal{F} \rightarrow \mathcal{F}$ is an endomorphism, we drop $\bar{C}$ from the notation and we write $C_{!}\left(j_{U !} \mathcal{F}, j_{!} u\right) \in H^{0}\left(X, j_{U !} \mathcal{K}_{U}\right)$. Further, if $u$ is the identity, we simply write $C_{!}\left(j_{U !} \mathcal{F}\right)$ and call it the refined characteristic class of $j_{U !} \mathcal{F}$.

It is clear from Proposition 2.1.7 that the characteristic class $C\left(j_{U !} \mathcal{F}, \bar{C}, j_{!} u\right) \in$ $H_{\bar{C} \cap X}^{0}\left(X, \mathcal{K}_{X}\right)$ is the image of the refinement $C_{!}\left(j_{U !} \mathcal{F}, \bar{C}, j_{!} u\right) \in H_{\bar{C} \cap X}^{0}\left(X, j_{U !} \mathcal{K}_{U}\right)$ by the canonical map $H_{\bar{C} \cap X}^{0}\left(X, j_{U !} \mathcal{K}_{U}\right) \rightarrow H_{\bar{C} \cap X}^{0}\left(X, \mathcal{K}_{X}\right)$. 
We give a compatibility with the pull-back. Let

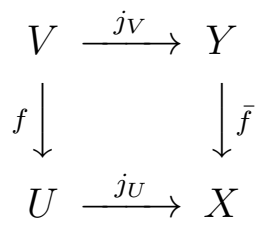

be a cartesian square of schemes over $k$ where the horizontal arrows are open immersions. We assume that $U$ and $V$ are smooth over $k$ and $\operatorname{dim} U=\operatorname{dim} V$. By this assumption, the map (1.9) induces a canonical map

$$
\bar{f}^{*} j_{U !} \mathcal{K}_{U}=j_{V !} f^{*} \mathcal{K}_{U} \rightarrow j_{V !} f^{!} \mathcal{K}_{U}=j_{V !} \mathcal{K}_{V}
$$

Let $c=\left(c_{1}, c_{2}\right): C \rightarrow U \times U$ be a closed immersion. Let $\bar{C}$ be the closure of $C$ in $X \times X$ and put $\bar{C}^{\prime}=(\bar{f} \times \bar{f})^{-1} \bar{C}$. Then, the map (2.6) induces a map $\bar{f}^{*}: H_{\bar{C} \cap X}^{0}\left(X, j_{U !} \mathcal{K}_{U}\right) \rightarrow$ $H_{\bar{C}^{\prime} \cap Y}^{0}\left(Y, j_{V !} \mathcal{K}_{V}\right)$.

Proposition 2.1.9 Let the notation be as above and assume that $C=\bar{C} \cap(X \times U)$. Let $\mathcal{F}$ be an object of $D_{\text {ctf }}(U)$ and let $u: c_{2}^{*} \mathcal{F} \rightarrow c_{1}^{!} \mathcal{F}$ be a cohomological correspondence on $C$. Let $j: C \rightarrow \bar{C}$ and $j^{\prime}: C^{\prime}=(f \times f)^{-1} C \rightarrow \bar{C}^{\prime}$ denote the open immersions. Then, we have $C^{\prime}=\bar{C}^{\prime} \cap(Y \times V)$ and

$$
C_{!}\left(j_{V !} f^{*} \mathcal{F}, \bar{C}^{\prime}, j_{!}^{\prime}(f \times f)^{*} u\right)=\bar{f}^{*} C_{!}\left(j_{U !} \mathcal{F}, \bar{C}, j_{!} u\right)
$$

in $H_{\bar{C}^{\prime} \cap Y}^{0}\left(Y, j_{V !} \mathcal{K}_{V}\right)$.

Proof. The equality $C^{\prime}=\bar{C}^{\prime} \cap(Y \times V)$ is clear from $C=\bar{C} \cap(X \times U)$. We put $\mathcal{H}=R \mathcal{H} o m\left(\operatorname{pr}_{2}^{*} \mathcal{F}, \operatorname{pr}_{1}^{!} \mathcal{F}\right)$ and $\overline{\mathcal{H}}=R \mathcal{H} o m\left(\operatorname{pr}_{2}^{*} j_{U !} \mathcal{F}, \operatorname{pr}_{1}^{!} j_{U !} \mathcal{F}\right)$ on $U \times U$ and on $X \times X$ respectively. We also put $\mathcal{H}^{\prime}=R \mathcal{H o m}\left(\operatorname{pr}_{2}^{*} f^{*} \mathcal{F}, \operatorname{pr}_{1}^{!} f^{*} \mathcal{F}\right)$ on $V \times V$ and $\overline{\mathcal{H}}^{\prime}=$ $R \mathcal{H} o m\left(\operatorname{pr}_{2}^{*} j_{V !} f^{*} \mathcal{F}, \operatorname{pr}_{1}^{!} j_{V !} f^{*} \mathcal{F}\right)$ on $Y \times Y$. We consider the commutative diagram

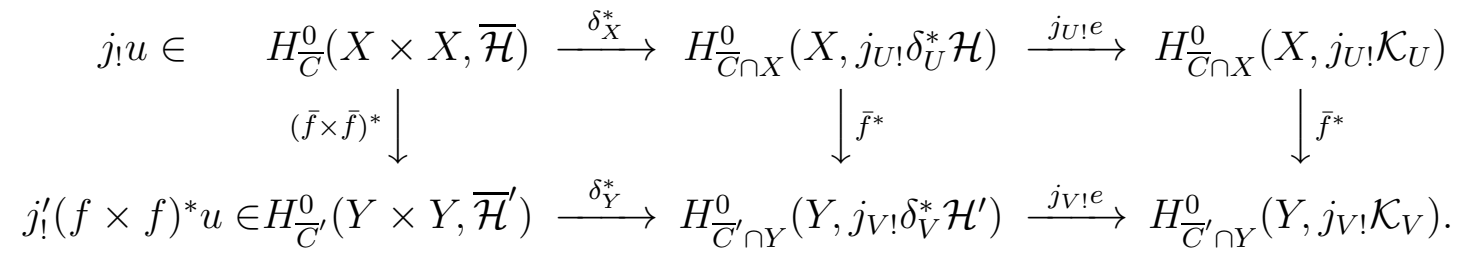

By the uniqueness Lemma 1.2.3, we have $(\bar{f} \times \bar{f})^{*} j_{!} u=j_{!}^{\prime}(f \times f)^{*} u$. Thus the assertion follows.

Corollary 2.1.10 If $X=U$ and $Y=V$, we have

$$
C\left(f^{*} \mathcal{F}, C^{\prime},(f \times f)^{*} u\right)=f^{*} C(\mathcal{F}, C, u) .
$$


We apply Proposition 2.1.9 to a finite étale Galois covering. We keep the notation in Proposition 2.1.9 and assume that $V \rightarrow U$ is a finite étale Galois covering of Galois group $G$. We assume further that $C=U$ is the diagonal and hence $u: \mathcal{F} \rightarrow \mathcal{F}$ is an endomorphism. For $\sigma \in G$, let $\Gamma_{\sigma} \subset V \times V$ be the graph of $\sigma: V \rightarrow V$ and let $\sigma^{*}: \sigma^{*} f^{*} \mathcal{F} \rightarrow(f \circ \sigma)^{*} \mathcal{F}=f^{*} \mathcal{F}$ denote the canonical map. We consider the composition $f^{*}(u) \circ \sigma^{*}: \sigma^{*} f^{*} \mathcal{F} \rightarrow f^{*} \mathcal{F}$ as a cohomological correspondence on $f^{*} \mathcal{F}$ on the graph $\Gamma_{\sigma} \subset V \times V$.

Corollary 2.1.11 Let the notation be as in Proposition 2.1.9. We assume that $C=$ $\Delta_{U} \subset U \times U$ and $u: \mathcal{F} \rightarrow \mathcal{F}$ is an endomorphism of $\mathcal{F}$. We assume further that $V \rightarrow U$ is a finite étale Galois covering of Galois group $G$. For $\sigma \in G$, let $j_{\sigma}: \Gamma_{\sigma} \rightarrow \overline{\Gamma_{\sigma}}$ be the open immersion to the closure in $Y \times Y$. Then, we have

$$
\bar{f}^{*} C_{!}\left(j_{U !} \mathcal{F}, j_{!} u\right)=\sum_{\sigma \in G} C_{!}\left(j_{V !} f^{*} \mathcal{F}, \overline{\Gamma_{\sigma}}, j_{\sigma !}\left(f^{*}(u) \circ \sigma^{*}\right)\right)
$$

in $H^{0}\left(Y, j_{V !} \mathcal{K}_{V}\right)$.

Proof. By Proposition 2.1.9, it suffices to show $C_{!}\left(j_{V !} f^{*} \mathcal{F}, \bar{C}^{\prime}, j_{!}^{\prime}(f \times f)^{*} u\right)=$ $\sum_{\sigma \in G} C_{!}\left(j_{V !} f^{*} \mathcal{F}, \overline{\Gamma_{\sigma}}, j_{\sigma !}\left(f^{*}(u) \circ \sigma^{*}\right)\right)$. Since $V \rightarrow U$ is an étale Galois covering, we have $(f \times f)^{*} u=\sum_{\sigma \in G} f^{*}(u) \circ \sigma^{*}$. Since $\overline{\Gamma_{\sigma}} \subset \bar{C}^{\prime}$, we have $C_{!}\left(j_{V !} f^{*} \mathcal{F}, \bar{C}^{\prime}, j_{!}^{\prime}\left(f^{*}(u) \circ \sigma^{*}\right)\right)=$ $C_{!}\left(j_{V !} f^{*} \mathcal{F}, \overline{\Gamma_{\sigma}}, j_{\sigma !}\left(f^{*}(u) \circ \sigma^{*}\right)\right)$ in $H^{0}\left(Y, j_{V !} \mathcal{K}_{V}\right)$ and the assertion follows.

Proposition 2.1.12 We consider a commutative diagram

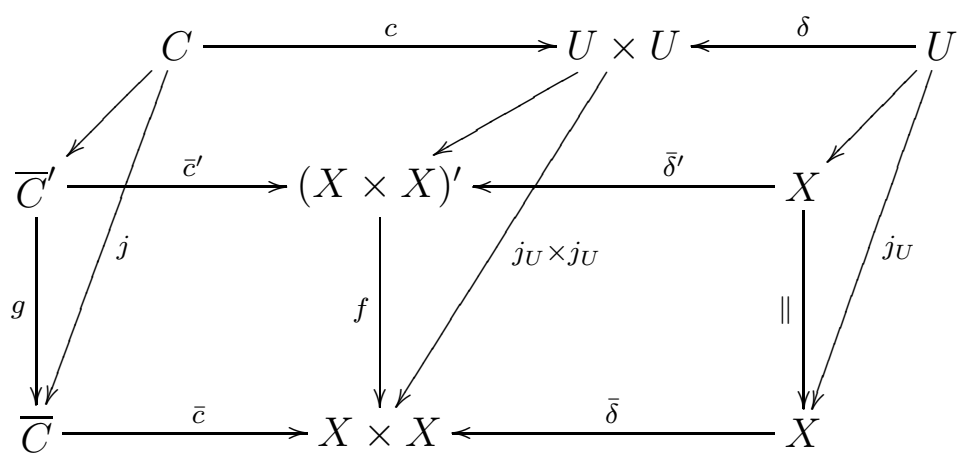

of schemes over $k$. We assume that the horizontal arrows are closed immersions, that the six slant arrows are open immersions, that the vertial arrows $f$ and $g$ are proper and that $f$ is an isomorphism on $(X \times U) \cup(U \times X)$. We also assume that the four slant parallelograms are cartesian and $C=\bar{C} \times_{X \times X}(X \times U)$.

Let $\mathcal{F}$ be an object of $D_{\mathrm{ctf}}(U) . \quad$ We put $\mathcal{H}=R \mathcal{H}$ om $\left(\mathrm{pr}_{2}^{*} \mathcal{F}, \mathrm{pr}_{1}^{!} \mathcal{F}\right)$ and $\mathcal{H}^{*}=$ $R \mathcal{H}$ om $\left(\operatorname{pr}_{1}^{*} \mathcal{F}, \operatorname{pr}_{2}^{!} \mathcal{F}\right)$ on $U \times U$. We also put $\overline{\mathcal{H}}=R \mathcal{H} o m\left(\operatorname{pr}_{2}^{*} j_{!} \mathcal{F}, \operatorname{pr}_{1}^{!} j_{!} \mathcal{F}\right)$ and $\overline{\mathcal{H}}^{*}=$ $R \mathcal{H}$ om $\left(\mathrm{pr}_{1}^{*} j_{!} \mathcal{F}, \mathrm{pr}_{2}^{!} j_{!} \mathcal{H}\right)$ on $X \times X$.

Let $\overline{\mathcal{H}}^{\prime}$ and $\overline{\mathcal{H}}^{* \prime}$ be objects of $D_{\text {ctf }}\left((X \times X)^{\prime}\right)$ and $a:\left.\left.\overline{\mathcal{H}}\right|_{U \times Y} \rightarrow \overline{\mathcal{H}}^{\prime}\right|_{U \times Y}$ and $b:\left.\overline{\mathcal{H}}^{*}\right|_{X \times V} \rightarrow$ $\left.\overline{\mathcal{H}}^{* \prime}\right|_{X \times V}$ be isomorphisms such that the unique maps $\bar{a}: \overline{\mathcal{H}} \rightarrow f_{*} \overline{\mathcal{H}}^{\prime}$ and $\bar{b}: \overline{\mathcal{H}}^{*} \rightarrow f_{*} \overline{\mathcal{H}}^{* \prime}$ 
inducing $a$ and $b$ in Proposition 1.3 .31 are isomorphisms. Let $\overline{\mathcal{H}}^{\prime} \otimes \overline{\mathcal{H}}^{* \prime} \rightarrow \mathcal{K}_{(X \times X)^{\prime}}$ be a map making the diagram (1.35) commutative.

Let $u: c_{!} \Lambda \rightarrow \mathcal{H}$ be a cohomological correspondence. Let $\bar{u}^{\prime}: \bar{c}_{!}^{\prime} \Lambda \rightarrow \overline{\mathcal{H}}^{\prime}$ and $\overline{1}^{\prime}: \bar{\delta}_{!}^{\prime} \Lambda \rightarrow$ $\overline{\mathcal{H}}^{\prime *}$ be maps making the diagrams (1.33) commutative for $\bar{d}=\bar{\delta}: X \rightarrow X \times X$ and $v=1$.

1. We have

$$
C\left(j_{!} \mathcal{F}, \bar{C}, j_{!} u\right)=\left\langle\bar{u}^{\prime}, \overline{1}^{\prime}\right\rangle
$$

in $H^{0}\left(\bar{C} \cap X, \mathcal{K}_{\bar{C} \cap X}\right)=H_{\bar{C} \cap X}^{0}\left(X, \mathcal{K}_{X}\right)$.

2. Further, we regard $\bar{u}^{\prime} \in H_{\bar{C}^{\prime}}^{0}\left((X \times X)^{\prime}, \overline{\mathcal{H}}^{\prime}\right)$ and let $\bar{\delta}^{\prime *}: H_{\bar{C}^{\prime}}^{0}\left((X \times X)^{\prime}, \overline{\mathcal{H}}^{\prime}\right) \rightarrow$ $H_{\bar{C}^{\prime} \cap X}^{0}\left(X, \bar{\delta}^{\prime *} \overline{\mathcal{H}}^{\prime}\right)$ be the pull-back. Let $\widetilde{\operatorname{Tr}}: \bar{\delta}^{\prime *} \overline{\mathcal{H}}^{\prime} \rightarrow K_{X}$ be the pairing $\bar{\delta}^{\prime *} \overline{\mathcal{H}}^{\prime} \otimes \bar{\delta}^{\prime} \cdot \overline{\mathcal{H}}^{* \prime} \rightarrow$ $\bar{\delta}^{\prime} K_{(X \times X)^{\prime}}=K_{X}$ (1.7) induced by $\overline{\mathcal{H}}^{\prime} \otimes \overline{\mathcal{H}}^{* \prime} \rightarrow K_{(X \times X)^{\prime}}$ evaluated at $\overline{1}^{\prime}$. Then, we have

$$
C(j ! \mathcal{F}, \bar{C}, j ! u)=\widetilde{\operatorname{Tr}} \bar{\delta}^{* *} \bar{u}^{\prime}
$$

in $H^{0}\left(\bar{C} \cap X, \mathcal{K}_{\bar{C} \cap X}\right)=H_{\bar{C} \cap X}^{0}\left(X, \mathcal{K}_{X}\right)$.

Proof. 1. By Corollary 1.2.4, we have $1=j ! 1$. Hence, we have $C(j ! \mathcal{F}, \bar{C}, j ! u)=$ $\left\langle j_{!} u, j_{!} 1\right\rangle$. Since the assumptions in Proposition 1.3 .3 are satisfied, we have $\left\langle j_{!} u, j_{!} 1\right\rangle=$ $f_{*}\left\langle\bar{u}^{\prime}, \overline{1}^{\prime}\right\rangle$. Since the restriction of $f$ to $X$ is the identity, the assertion follows.

2. We have $\left\langle\bar{u}^{\prime}, \overline{1}^{\prime}\right\rangle=\widetilde{\operatorname{Tr}} \delta^{*} \bar{u}^{\prime}$ by Lemma 1.3.1. Thus, it follows from 1 .

\subsection{Characteristic class and log blow-up}

In this subsection, $X$ denotes a smooth scheme of dimension $d$ over $k$ and $D=$ $\bigcup_{i=1}^{m} D_{i} \subset X$ denotes a divisor with simple normal crossings. Let $U \subset X$ be the complement of $D$ and $j: U \rightarrow X$ be the open immersion.

Let $(X \times X)^{\prime} \rightarrow X \times X$ be the blow-up at $D_{i} \times D_{i}$ for $i=1, \ldots, m$. It is defined by the product of the ideal sheaves $I_{D_{i} \times D_{i}} \subset O_{X \times X}$. Let $p_{1}, p_{2}:(X \times X)^{\prime} \rightarrow X$ be the projections. For $i=1, \ldots, m$, let $\left(D_{i} \times X\right)^{\prime}$ be the proper transform of $D_{i} \times X$ and $\left(X \times D_{i}\right)^{\prime}$ be that of $X \times D_{i}$. We put $D^{(1) \prime}=\bigcup_{i}\left(D_{i} \times X\right)^{\prime}$ and $D^{(2) \prime}=\bigcup_{i}\left(X \times D_{i}\right)^{\prime}$. We define open subschemes $(U \times X)^{\prime}$ and $(X \times U)^{\prime}$ of $(X \times X)^{\prime}$ to be the complements of $D^{(1) \prime}$ and of $D^{(2) \prime}$ respectively.

The intersection $(X \times X)^{\sim}=(U \times X)^{\prime} \cap(X \times U)^{\prime}$ is the maximum open subscheme of $(X \times X)^{\prime}$ where $p_{1}^{*} D_{i}=p_{2}^{*} D_{i}$ for each $i=1, \ldots, m$. We obtain a commutative 
diagram

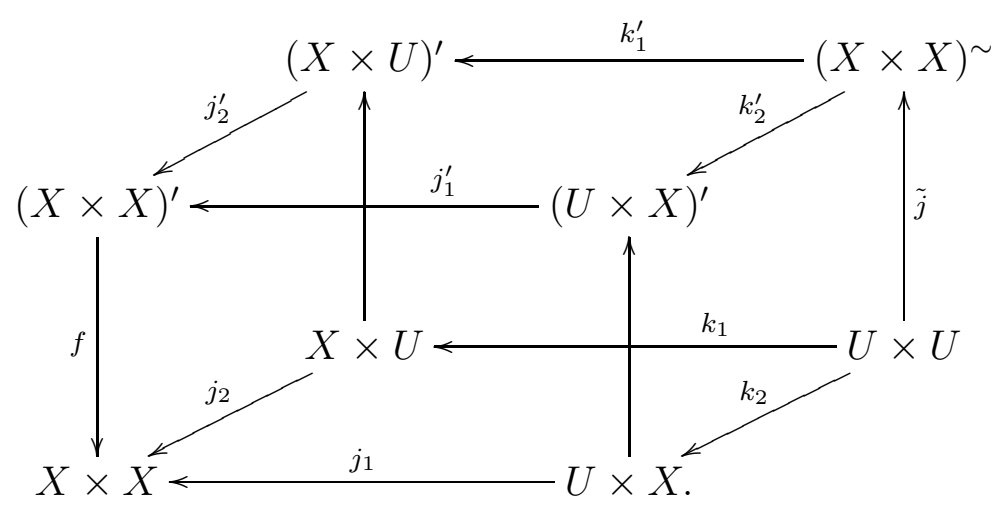

All the arrows except the blow-up $f:(X \times X)^{\prime} \rightarrow X \times X$ are open immersions. The four faces consisting of open immersions are cartesian. The diagonal map $\delta_{X}=\delta: X=$ $\Delta_{X} \rightarrow X \times X$ induces the log diagonal map $\delta_{X}^{\prime}=\delta^{\prime}: X=\Delta_{X}^{\log } \rightarrow(X \times X)^{\sim} \subset(X \times X)^{\prime}$.

Let $\mathcal{F}$ be a locally constant sheaf of free $\Lambda$-modules of finite rank on $U$ tamely ramified along $D=X \backslash U$. We put $\mathcal{H}_{0}=\mathcal{H o m}\left(\mathrm{pr}_{2}^{*} \mathcal{F}, \operatorname{pr}_{1}^{*} \mathcal{F}\right)$ and $\mathcal{H}=R \mathcal{H} o m\left(\operatorname{pr}_{2}^{*} \mathcal{F}, \operatorname{pr}_{1}^{!} \mathcal{F}\right)=$ $\mathcal{H}_{0}(d)[2 d]$ on $U \times U$. We put $\overline{\mathcal{H}}=R \mathcal{H} o m\left(\operatorname{pr}_{2}^{*} j_{!} \mathcal{F}, \operatorname{pr}_{1}^{!} j_{!} \mathcal{F}\right)=j_{1 !} R k_{2 *} \mathcal{H}=R j_{2 *} k_{1 !} \mathcal{H}$ on $X \times X$. We put $\widetilde{\mathcal{H}}_{0}=\tilde{j}_{*} \mathcal{H}_{0}$ on $(X \times X)^{\sim}$. Here and in the rest of this subsection, the symbol $j_{*}$ for an open immersion $j$ denotes the usual direct image and is not an abbreviation of $R j_{*}$. We also put $\widetilde{\mathcal{H}}=\widetilde{\mathcal{H}}_{0}(d)[2 d]$. The trace map $\operatorname{Tr}: \delta_{U}^{*} \mathcal{H}_{0}=\mathcal{E} n d(\mathcal{F}) \rightarrow \Lambda_{U}$ is extended uniquely to a map

$$
\widetilde{\operatorname{Tr}}: \delta^{*} \widetilde{\mathcal{H}}_{0}=j_{*} \mathcal{E} n d(\mathcal{F}) \longrightarrow \Lambda_{X}
$$

We define $\overline{\mathcal{H}}^{\prime}=j_{1 !}^{\prime} R k_{2 *}^{\prime} \widetilde{\mathcal{H}}=R j_{2 *}^{\prime} k_{1 !}^{\prime} \widetilde{\mathcal{H}}$ on $(X \times X)^{\prime}$.

Definition 2.2.1 Let $j: U \rightarrow X$ and $\mathcal{F}$ on $U$ be as above. Let $C$ be a closed subscheme of $U \times U$ and let $\bar{C}^{\prime}$ and $\widetilde{C}=\bar{C}^{\prime} \cap(X \times X)^{\sim}$ be the closures of $C$ in $(X \times X)^{\prime}$ and in $(X \times X)^{\sim}$ respectively.

1. We say that a closed subscheme $C \subset U \times U$ is non-expanding with respect to $X$ if we have $\widetilde{C}=\bar{C}^{\prime} \cap(X \times U)^{\prime}$.

2. Let $C \subset U \times U$ be a closed subscheme that is non-expanding with respect to $X$. We call the image of the injection $\Gamma\left(\widetilde{C},\left.\widetilde{\mathcal{H}}_{0}\right|_{\widetilde{C}}\right) \rightarrow \Gamma\left(C,\left.\mathcal{H}_{0}\right|_{C}\right)$ the tame part of $\Gamma\left(C,\left.\mathcal{H}_{0}\right|_{C}\right)=\operatorname{Hom}\left(c_{2}^{*} \mathcal{F}, c_{1}^{*} \mathcal{F}\right)$.

3. Let $\gamma: c_{2}^{*} \mathcal{F} \rightarrow c_{1}^{*} \mathcal{F}$ be a map in the tame part. For a cycle class $\widetilde{\Gamma} \in C H_{d}(\widetilde{C})$, let $\tilde{u}=\tilde{u}(\widetilde{\Gamma}, \gamma) \in H_{\widetilde{C}}^{0}\left((X \times X)^{\sim}, \widetilde{\mathcal{H}}\right)$ denote the cup-product of $\operatorname{cl}(\widetilde{\Gamma}) \in H_{\widetilde{C}}^{0}((X \times$ $\left.X)^{\sim}, \Lambda(d)[2 d]\right)$ with $\gamma \in \Gamma\left(\widetilde{C}, \widetilde{\mathcal{H}}_{0}\right)$. Let $\widetilde{\operatorname{Tr}}\left(\delta^{\prime *} \gamma\right) \in \Gamma(\widetilde{C} \cap X, \Lambda)$ denote the image of $\delta^{* *} \gamma \in \Gamma\left(\widetilde{C} \cap X, \widetilde{\mathcal{H}}_{0}\right)$ by the map induced by $\widetilde{\operatorname{Tr}}(2.9)$.

If a closed subscheme $C \subset U \times U$ is non-expanding with respect to $X$, the closure $\bar{C} \subset X \times X$ of $C$ satisfies $\bar{C} \cap(X \times U)=\widetilde{C} \cap(X \times U)=C$. For $\widetilde{\Gamma} \in C H_{d}(\widetilde{C})$ and 
$\gamma: c_{2}^{*} \mathcal{F} \rightarrow c_{1}^{*} \mathcal{F}$ in the tame part, the element $\tilde{u}=\tilde{u}(\widetilde{\Gamma}, \gamma) \in H_{\widetilde{C}}^{0}\left((X \times X)^{\sim}, \widetilde{\mathcal{H}}\right)$ is an extension of $u=u(\Gamma, \gamma) \in H_{C}^{0}(U \times U, \mathcal{H})$ defined by (1.15).

The following lemma gives an example of a non-expanding closed subscheme.

Lemma 2.2.2 (16] Proposition 1.1.6) Let $j: U \rightarrow X$ be as above. We consider a cartesian diagram

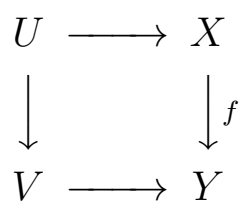

of morphisms of schemes over $k$. We assume $V$ is the complement of a Cartier divisor $B$ of $Y$. Then, the closed subscheme $C=U \times{ }_{V} U \subset U \times U$ is non-expanding with respect to $X$.

In the tame case, the characteristic class is computed as follows.

Proposition 2.2.3 Let the notation be as above. Let $\mathcal{F}$ be a smooth $\Lambda$-sheaf on $U=$ $X \backslash D$ tamely ramified along $D$. Let $C \subset U \times U$ be a closed subscheme that is nonexpanding with respect to $X$ and $\bar{C} \subset X \times X, \bar{C}^{\prime} \subset(X \times X)^{\prime}$ and $\widetilde{C} \subset(X \times X)^{\sim}$ be the closures.

1. The restriction map

$$
H_{\bar{C}^{\prime}}^{0}\left((X \times X)^{\prime}, \overline{\mathcal{H}}^{\prime}\right) \rightarrow H_{\widetilde{C}}^{0}\left((X \times X)^{\sim}, \widetilde{\mathcal{H}}\right)
$$

is an isomorphism.

2. Let $\gamma: c_{2}^{*} \mathcal{F} \rightarrow c_{1}^{*} \mathcal{F}$ be an element in the tame part $\Gamma\left(\widetilde{C},\left.\widetilde{\mathcal{H}}_{0}\right|_{\widetilde{C}}\right) \subset \Gamma\left(C,\left.\mathcal{H}_{0}\right|_{C}\right)=$ $\operatorname{Hom}\left(c_{2}^{*} \mathcal{F}, c_{1}^{*} \mathcal{F}\right)$. Let $\widetilde{\Gamma} \in C H_{0}(\widetilde{C})$ be an algebraic cycle class and $\Gamma \in C H_{0}(C)$ be the restriction. Then, we have

$$
C\left(j ! \mathcal{F}, \bar{C}, j_{!} u(\Gamma, \gamma)\right)=\operatorname{cl}\left(\widetilde{\Gamma}, \Delta_{X}^{\log }\right)_{(X \times X) \sim} \cdot \widetilde{\operatorname{Tr}} \delta^{*} \gamma
$$

in $H_{\bar{C} \cap X}^{2 d}(X, \Lambda(d))=H_{\bar{C} \cap X}^{0}\left(X, \mathcal{K}_{X}\right)$. The right hand side is the product of the class $\operatorname{cl}\left(\widetilde{\Gamma}, \Delta_{X}^{\log }\right)_{(X \times X) \sim} \in H_{\bar{C} \cap X}^{0}\left(X, \mathcal{K}_{X}\right)$ of the intersection product and $\widetilde{\operatorname{Tr}} \delta^{*} \gamma \in \Gamma(\bar{C} \cap X, \Lambda)$ defined in Definition 2.2.1.3.

Proof. 1. Since $\bar{C}^{\prime} \cap(X \times U)^{\prime}=\widetilde{C}$, we have an isomorphism

$$
\begin{aligned}
& H_{\bar{C}^{\prime}}^{0}\left((X \times X)^{\prime}, \overline{\mathcal{H}}^{\prime}\right)=H_{\bar{C}^{\prime}}^{0}\left((X \times X)^{\prime}, R j_{2 *}^{\prime} k_{1 !}^{\prime} \widetilde{\mathcal{H}}\right) \\
\rightarrow \quad & H_{\bar{C}^{\prime}}^{0}{ }_{(X \times U)^{\prime}}\left((X \times U)^{\prime}, k_{1 !}^{\prime} \widetilde{\mathcal{H}}\right)=H_{\widetilde{C}}^{0}\left((X \times X)^{\sim}, \widetilde{\mathcal{H}}\right)
\end{aligned}
$$

as in (1.22). 
2. Similarly as $\overline{\mathcal{H}}^{\prime}=j_{1 !}^{\prime} R k_{2 *}^{\prime} \tilde{j}_{*} \mathcal{H}_{0}(d)[2 d]$, we put $\mathcal{H}_{0}^{*}=\mathcal{H} o m\left(\operatorname{pr}_{1}^{*} \mathcal{F}, \operatorname{pr}_{2}^{*} \mathcal{F}\right)$ on $U \times U$, $\widetilde{\mathcal{H}}^{*}=\tilde{j}_{*} \mathcal{H}_{0}^{*}(d)[2 d]$ on $(X \times X)^{\sim}$, and $\overline{\mathcal{H}}^{\prime *}=j_{2 !}^{\prime} R k_{1 *}^{\prime} \widetilde{\mathcal{H}}^{*}=R j_{1 *}^{\prime} k_{2 !}^{\prime} \widetilde{\mathcal{H}}^{*}$ on $(X \times X)^{\prime}$. By 1 , we regard $\tilde{u}(\tilde{\Gamma}, \gamma) \in H_{\widetilde{C}}^{0}\left((X \times X)^{\sim}, \widetilde{\mathcal{H}}\right)$ and $\tilde{u}(X, 1) \in H_{X}^{0}\left((X \times X)^{\sim}, \widetilde{\mathcal{H}}^{* \prime}\right)$ as elements of $H_{\bar{C}^{\prime}}^{0}\left((X \times X)^{\prime}, \overline{\mathcal{H}}^{\prime}\right)$ and $H_{X}^{0}\left((X \times X)^{\prime}, \overline{\mathcal{H}}^{* \prime}\right)$ respectively. We consider a pairing $\overline{\mathcal{H}}^{\prime} \otimes \overline{\mathcal{H}}^{\prime *}=\left(j_{1}^{\prime} k_{2}^{\prime}\right)_{!}\left(\tilde{j}_{*} \mathcal{H}_{0} \otimes \tilde{j}_{*} \mathcal{H}_{0}^{*}\right)(2 d)[4 d] \rightarrow \mathcal{K}_{(X \times X)^{\prime}}$ induced by the pairing $\mathcal{H}_{0} \otimes \mathcal{H}_{0}^{*} \rightarrow \Lambda_{U \times U}: a \otimes b \mapsto \operatorname{Tr}(b \circ a)$.

By Lemma 2.2.4 below, the assumptions in Proposition 2.1.12 are satisfied. We claim that the map $\widetilde{\operatorname{Tr}}: \bar{\delta}^{\prime *} \overline{\mathcal{H}}^{\prime}=j_{*} \mathcal{E} n d(\mathcal{F})(d)[2 d] \rightarrow K_{X}=\Lambda_{X}(d)[2 d]$ in Proposition 2.1.12, 2 is induced by the map $\widetilde{T r}$ defined in (2.9). On $U$, it is shown in the proof of Proposition 2.1.7. Since the map $\widetilde{\operatorname{Tr}}: \delta^{*} \widetilde{\mathcal{H}}_{0}=j_{*} \mathcal{E} n d(\mathcal{F}) \rightarrow \Lambda_{X}$ is the unique map extending $\operatorname{Tr}: \delta^{*} \mathcal{H}_{0}=\mathcal{E} n d(\mathcal{F}) \rightarrow \Lambda_{U}$, it induces the map $\widetilde{\operatorname{Tr}}: \bar{\delta}^{*} \overline{\mathcal{H}}^{\prime} \rightarrow K_{X}$. Thus, applying Proposition 2.1.12 2, we obtain $C\left(j_{!} \mathcal{F}, \bar{C}, j_{!} u\right)=\widetilde{\operatorname{Tr}} \delta^{*} \tilde{u}(\tilde{\Gamma}, \gamma)=\delta^{*}(\operatorname{cl}(\tilde{\Gamma}))$. $\widetilde{\operatorname{Tr}} \delta^{*} \gamma$. By the compatibility (1.11), we have $\delta^{\prime *}(\operatorname{cl}(\tilde{\Gamma}))=\operatorname{cl}\left(\tilde{\Gamma}, \Delta_{X}^{\log }\right)_{(X \times X) \sim}$. Thus the assertion follows.

Lemma 2.2.4 (cf. [8], [17], 16] Lemma 2.2.1) The canonical maps $\overline{\mathcal{H}} \rightarrow f_{*} \overline{\mathcal{H}}^{\prime}$ and $\overline{\mathcal{H}}^{*} \rightarrow f_{*} \overline{\mathcal{H}}^{* \prime}$ are isomorphisms.

Proof. It suffices to show that the stalk of $f_{*} \overline{\mathcal{H}}^{\prime}$ is 0 at an arbitrary geometric point of $\bigcup_{i} D_{i} \times D_{i}$. Let $x \in \bigcup_{i} D_{i} \times D_{i}$ and $D_{1}, \ldots, D_{m}$ be the components of $D$. Then, since the question is étale local and since it is reduced to the case where $\Lambda$ is a field, we may assume $\mathcal{F}$ is the tensor product $\mathcal{F}_{1} \otimes \cdots \otimes \mathcal{F}_{m}$ where $\mathcal{F}_{i}$ is the extension by zero of a smooth sheaf of rank 1 on the complement $X \backslash D_{i}$ for each $i=1, \ldots, r$.

Let $(X \times X)_{i}^{\prime}$ be the blow-up of $X \times X$ at $D_{i} \times D_{i}$. Then, $(X \times X)^{\prime}$ is the fiber product of $(X \times X)_{i}^{\prime}$ over $X \times X$. Hence, by the Künneth formula, it is reduced to the case $r=1$ and $D=D_{1}$.

The fiber of $(X \times X)^{\sim} \subset(X \times X)^{\prime} \rightarrow X \times X$ at a point in $D_{1} \times D_{1}$ is isomorphic to $\mathbb{G}_{m} \subset \mathbf{P}^{1}$ and the restriction of $\mathcal{H}_{0}$ to $\mathbb{G}_{m}$ is smooth and is tamely ramified along 0 and $\infty$. Hence, by the proper base change theorem, it is reduced to showing that $H^{q}\left(\mathbb{A} \frac{1}{k}, j ! \mathcal{G}\right)=0$ for $q=0,1,2$ for a smooth sheaf $\mathcal{G}$ on $\mathbb{G}_{m}$ tamely ramified at 0 and $\infty$ where $j: \mathbb{G}_{m} \rightarrow \mathbb{A}^{1}$ is the open immersion.

It is clear for $q=0$ and follows by duality for $q=2$. The smooth sheaf $\mathcal{G}$ is trivialized by the covering $[m]: \mathbb{G}_{m} \rightarrow \mathbb{G}_{m}$ for an integer $m$ invertible in $k$. Thus, the case $q=1$ is reduced to the case where $\mathcal{G}$ is constant. Hence it follows from the acyclicity of $\mathbb{A}^{1}$.

We put $C_{U}=(-1)^{d} c_{d}\left(\Omega_{X / k}^{1}(\log D)\right) \in H^{2 d}\left(X, \mathbb{Z}_{\ell}(d)\right)$. Since the conormal sheaf $N_{X /(X \times X) \sim}$ is canonically isomorphic to $\Omega_{X / k}^{1}(\log D)$, we have $C_{U}=\left(\Delta_{X}^{\log }, \Delta_{X}^{\log }\right)_{(X \times X)^{\sim}}$.

Corollary 2.2.5 1. Assume that $\mathcal{F}$ is tamely ramified along $D$ and that $C=U$ is the diagonal. Then every endomorphism of $\mathcal{F}$ is in the tame part and we have $\operatorname{End}_{U}(\mathcal{F})^{\sim}=\operatorname{End}_{U}(\mathcal{F})$. For an endomorphism $\gamma \in \operatorname{End}_{U}(\mathcal{F})$, we have

$$
C(j ! \mathcal{F}, j ! \gamma)=\operatorname{Tr} \gamma \cdot C_{U}
$$


in $H^{2 d}(X, \Lambda(d))$. In particular, for $\mathcal{F}=\Lambda$ and $\gamma=\mathrm{id}$, we have

$$
C(j ! \Lambda)=C_{U}
$$

2. Assume $C$ is non-expanding and is of dimension d. Then, we have

$$
C\left(j_{!} \Lambda, C, u(C, 1)\right)=\left(\widetilde{C}, \Delta_{X}^{\log }\right)_{(X \times X) \sim} .
$$

If $X$ is proper, taking the trace of (2.13), we recover the Lefschetz trace formula

$$
\operatorname{Tr}\left(C^{*}, H_{c}^{*}\left(U_{\bar{k}}, \Lambda\right)\right)=\operatorname{Tr}\left(\widetilde{C}, \Delta_{X}^{\log }\right)_{(X \times X)^{\sim}}
$$

for an open variety [16] Theorem 2.3.4. In particular, if $C=U$, we have

$$
\chi_{c}(U)=\operatorname{deg} C_{U}=\operatorname{deg}\left(\Delta_{X}^{\log }, \Delta_{X}^{\log }\right)_{(X \times X)^{\sim}} .
$$

\section{Characteristic class and Swan class}

In this section, $\ell$ denotes a prime number invertible on a noetherian scheme $X$ and $E$ denotes a finite extension of $\mathbb{Q}_{\ell}$. Let $O$ be the integer ring of $E$ and $F=O / \lambda$ be the residue field of $E$. For a constructible $E$-sheaf $\mathcal{F}$ on $X, \mathcal{F}_{O}$ denotes an $O$-lattice and $\mathcal{F}_{n}$ denotes the reduction $\mathcal{F}_{O} \otimes_{O} O / \lambda^{n}$. We put $\overline{\mathcal{F}}=\mathcal{F}_{1}$.

For a constructible $E$-sheaf $\mathcal{F}$ on a scheme $X$ over $k$ and a cohomological correspondence $u$ on $C \subset X \times X$, the characteristic class $C(\mathcal{F}, C, u) \in H^{0}\left(X, \mathcal{K}_{X}\right)$ is defined as $C\left(\mathcal{F}_{n}, C, u_{n}\right)_{n} \in \lim _{n} H^{0}\left(X, R f^{!} O / \lambda^{n}\right) \otimes_{O} E$. It inherits the properties established in the previous section for torsion coefficients. For a smooth $E$-sheaf $\mathcal{F}$ on a smooth dense open subscheme $U \subset X$, we will prove that the difference $C\left(j_{!} \mathcal{F}\right)-\operatorname{rank} \mathcal{F} \cdot C\left(j_{!} E\right)$ is given by the Swan class $\operatorname{Sw}(\mathcal{F})$ defined in 16, under a certain assumption formulated using a notion introduced in $\S 3.1$. The definition of the $\operatorname{Swan}$ class $\operatorname{Sw}(\mathcal{F})$ will be recalled in $\S 3.2$.

\section{$3.1 \quad \ell$-adic sheaves potentially of Kummer type}

Definition 3.1.1 Let $X$ be a scheme and $U \subset X$ be an open subscheme.

1. Let $D_{i} \subset X, i \in I$ be a finite family of Cartier divisors such that $U \cap D_{i}=\emptyset$ for each $i \in I$. Then, we say a locally constant constructible sheaf $\mathcal{F}$ of sets on $U$ is of Kummer type with respect to $D_{i}, i \in I$ if the following condition is satisfied.

(Kum) For each $x \in X$, there exist an étale neighborhood $W \rightarrow X$ of $x$, bases $t_{i}$ of the invertible ideals $\left.O\left(-D_{i}\right)\right|_{W}$ and an integer $m$ invertible on $W$ such that the pull-back of $\mathcal{F}$ is constant on $U \times_{X} W\left[T_{i}, i \in I\right] /\left(T_{i}^{m}-t_{i}, i \in I\right)$.

We say that a finite étale scheme $V$ over $U$ is of Kummer type with respect to $D_{i}, i \in I$ if the locally constant sheaf on $U$ represented by $V$ is of Kummer type with respect to $D_{i}, i \in I$. 
2. We say that a locally constant constructible sheaf $\mathcal{F}$ of sets on $U$ is of Kummer type with respect to $X$ if there exists a finite family of Cartier divisors $D_{i} \subset X, i \in I$ such that $\mathcal{F}$ is of Kummer type with respect to $D_{i}, i \in I$. We say that a smooth $E$-sheaf $\mathcal{F}$ on $U$ is of Kummer type with respect to $X$ if there exists a finite family of Cartier divisors $D_{i} \subset X, i \in I$ such that $\mathcal{F}_{n}$ is of Kummer type with respect to $D_{i}, i \in I$ for every $n \geq 0$.

3. We say that a smooth E-sheaf $\mathcal{F}$ on $U$ is potentially of Kummer type with respect to $X$ if there exists a cartesian diagram

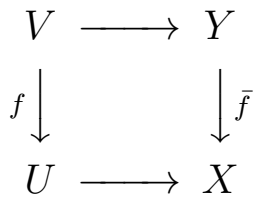

satisfying the following properties: The map $\bar{f}: Y \rightarrow X$ is proper surjective, $f: V \rightarrow U$ is finite étale and $f^{*} \mathcal{F}$ is of Kummer type with respect to $Y$.

For a geometric point $\bar{x}$ of a normal noetherian scheme $X$, let $X_{\bar{x}}$ be the strict localization of $X$ at $\bar{x}$ and let $I_{\bar{x}}=\pi_{1}\left(U \times_{X} X_{\bar{x}}, \bar{\eta}_{x}\right)$ be the inertia group at $\bar{x}$ where $\bar{\eta}_{\bar{x}}$ is a geometric point of $U_{\bar{x}}=U \times_{X} X_{\bar{x}}$.

Lemma 3.1.2 Let $X$ be a normal noetherian scheme and $U \subset X$ be an open subscheme. We consider the following conditions on a locally constant constructible sheaf $\mathcal{F}$ of sets on $U$.

(1) $\mathcal{F}$ is of Kummer type with respect to $X$.

(2) For each geometric point $\bar{x}$ of $X$, the image of $I_{\bar{x}} \rightarrow \operatorname{Aut}\left(\mathcal{F}_{\bar{\eta}_{\bar{x}}}\right)$ is a finite abelian group of order invertible at $\bar{x}$.

(3) For each geometric point $\bar{x}$ of $X$ such that $O_{X, \bar{x}}$ is a discrete valuation ring, the image of $I_{\bar{x}} \rightarrow \operatorname{Aut}\left(\mathcal{F}_{\bar{\eta}_{\bar{x}}}\right)$ is a finite group of order invertible at $\bar{x}$.

Then, we have (1) $\Rightarrow$ (2) $\Rightarrow$ (3).

If $X$ is a regular noetherian scheme and $U$ is the complement of a divisor with simple normal crossings, then the three conditions (1), (2) and (3) are equivalent.

Proof. (1) $\Rightarrow(2)$ Let $\mathcal{F}$ be a locally constant constructible sheaf of sets on $U$ that is of Kummer type with respect to $D_{i}, i \in I$. Let the notation be as in the condition (Kum) and take a geometric point $\bar{x}$ above $x$. We define a map

$$
I_{\bar{x}} \rightarrow \prod_{i \in I} \mu_{m}
$$

by sending $\sigma$ to $\left(\sigma\left(\sqrt[m]{t_{i}}\right) / \sqrt[m]{t_{i}}\right)_{i}$. Then the image $I_{\bar{x}} \rightarrow \operatorname{Aut}\left(\mathcal{F}_{\bar{\eta}_{\bar{x}}}\right)$ is a quotient of the image of $I_{\bar{x}} \rightarrow \prod_{i} \mu_{m}$.

$(2) \Rightarrow(3)$ Clear.

$(3) \Rightarrow(1)$ We assume $X$ is regular and $D=X \backslash U$ is a divisor with simple normal crossings. Then $\mathcal{F}$ is of Kummer type with respect to the family $D_{i}, i \in I$ of the irreducible components of $D$ by [18] Proposition 5.2 (Lemme d'Abhyankhar absolu). 
Corollary 3.1.3 Let $X$ be a normal scheme over $k$ and $U \subset X$ be a dense open subscheme smooth over $k$. If $\operatorname{dim} X \leq 2$, an arbitrary smooth E-sheaf $\mathcal{F}$ on $U$ is potentially of Kummer type with respect to $X$.

Proof. Clear from Lemma 3.1.2 and the strong resolution of singularities for surfaces.

Remark. Let $U \subset X$ be a dense open subscheme of a normal scheme $X$ over $k$ and $\mathcal{F}$ be a locally constant constructible sheaf of sets on $U$. We consider the following conditions:

$\left(1^{\prime}\right)$ There exists a proper modification $X^{\prime} \rightarrow X$ such that $\mathcal{F}$ is of Kummer type with respect to $X^{\prime}$.

$\left(2^{\prime}\right)$ For each geometric point $\bar{x}$ of $X$, the image of the inertia $I_{\bar{x}} \rightarrow$ Aut $\mathcal{F}_{\bar{x}}$ has order invertible in $k$.

Kato has told the authors that $\left(2^{\prime}\right)$ implies $\left(1^{\prime}\right)$. For the other implication, there is a counterexample.

We consider a geometric construction generalizing the log blow-up studied in the previous section. Assume $k$ is perfect and let $Y$ be a normal scheme over $k$. Let $V \subset Y$ be an open subscheme and $j: V \rightarrow Y$ denote the open immersion. Let $D_{i}, i \in I$ be a finite family of Cartier divisors of $Y$ such that $V \cap D_{i}=\emptyset$. Let $(Y \times Y)^{\prime}$ be the blow-up of $Y \times Y$ at $D_{i} \times D_{i}$ for each $i \in I, \overline{(Y \times Y)^{\prime}}$ be its normalization and let $p_{1}, p_{2}: \overline{(Y \times Y)^{\prime}} \rightarrow Y$ denote the projections. Let $(Y \times Y)^{\sim} \subset \overline{(Y \times Y)^{\prime}}$ be the maximum open subscheme where $p_{1}^{*} D_{i}=p_{2}^{*} D_{i}$ for each $i \in I$. Let $\tilde{j}: V \times V \rightarrow(Y \times Y)^{\sim}$ denote the open immersion.

We consider an endomorphism $g: Y \rightarrow Y$ such that $g^{-1}(V)=V$. Let $\widetilde{Y}_{g} \subset Y$ be the maximum open subscheme where $D_{i}=g^{*} D_{i}$ for each $i \in I$. Then, by the universality of blow-up and by the assumption that $Y$ is normal, the immersion $\gamma=(1, g): Y \rightarrow Y \times Y$ induces an immersion $\tilde{\gamma}: \widetilde{Y}_{g} \rightarrow(Y \times Y)^{\sim}$. If $g$ is the identity of $Y$, we obtain an immersion $\tilde{\delta}: Y=\widetilde{Y}_{\text {id }} \rightarrow(Y \times Y)^{\sim}$. Thus, we have a commutative diagram

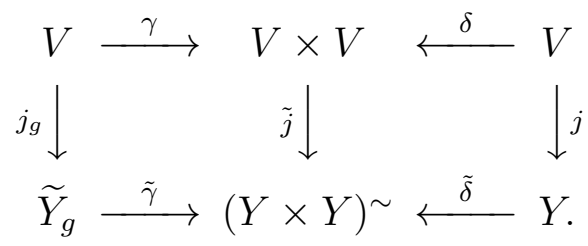

For a geometric point $\bar{y}$ of $Y$, let $Y_{\bar{y}}$ denote the strict localization. Taking a geometric point $\bar{\eta}$ of $V_{\bar{y}}=V \times_{Y} Y_{\bar{y}}$, we define the inertia group by $I_{\bar{y}}=\pi_{1}\left(V_{\bar{y}}, \bar{\eta}\right)$.

Proposition 3.1.4 Let the notation be as above. Let $\mathcal{F}$ and $\mathcal{G}$ be locally constant constructible sheaves of sets on $V$ of Kummer type with respect to $D_{i}, i \in I$ and put $\mathcal{H}=\mathcal{H o m}\left(\operatorname{pr}_{2}^{*} \mathcal{G}, \operatorname{pr}_{1}^{*} \mathcal{F}\right)$ on $V \times V$. 
1. The base change map

$$
\tilde{\gamma}^{*} \tilde{j}_{*} \mathcal{H} \longrightarrow j_{g *} \gamma^{*} \mathcal{H}
$$

is an isomorphism.

2. Let $y \in \widetilde{Y}_{g} \backslash V$ be a point such that $\tilde{\delta}(y)=\tilde{\gamma}(y)$. Let $\bar{y}$ be a geometric point above $y$ and let $I_{\bar{y}}=\pi_{1}\left(V_{\bar{y}}, \bar{\eta}\right)$ be the inertia group where $\bar{\eta}$ is a geometric point of $V_{\bar{y}}=$ $V \times_{Y} Y_{\bar{y}}$. We identify $\left(j_{*} \delta^{*} \mathcal{H}\right)_{\bar{y}}=\left(j_{*} \mathcal{H} \operatorname{Hom}(\mathcal{G}, \mathcal{F})\right)_{\bar{y}}=\operatorname{Hom}_{I_{\bar{y}}}\left(\mathcal{G}_{\bar{\eta}}, \mathcal{F}_{\bar{\eta}}\right)$ and $\left(j_{g *} \gamma^{*} \mathcal{H}\right)_{\bar{y}}=$ $\left(j_{g *} \mathcal{H o m}\left(g^{*} \mathcal{G}, \mathcal{F}\right)\right)_{\bar{y}}=\operatorname{Hom}_{I_{\bar{y}}}\left(g^{*} \mathcal{G}_{\bar{\eta}}, \mathcal{F}_{\bar{\eta}}\right)$. Let $m$ be an integer satisfying the property in the condition (Kum) for the sheaves $\mathcal{F}$ and $\mathcal{G}$.

We define $u_{i} \in O_{Y, \bar{y}}^{\times}$by $g^{*}\left(t_{i}\right)=u_{i} t_{i}$ for each $i \in I$. Then, we have $u_{i}(\bar{y})=1$. Let $v_{i} \in O_{Y, \bar{y}}^{\times}$be the unique $m$-th root of $u_{i}$ such that $v_{i}(\bar{y})=1$ for each $i \in I$. Let $\bar{g}: \bar{\eta} \rightarrow \bar{\eta}$ be a map compatible with $g: V \rightarrow V$ and assume $\bar{g}^{*}\left(\sqrt[m]{t_{i}}\right)=v_{i} \sqrt[m]{t_{i}}$ for each $i \in I$. Let $\bar{g}^{*}: g^{*} \mathcal{G}_{\bar{\eta}}=\bar{g}^{*}\left(\mathcal{G}_{\bar{\eta}}\right) \rightarrow \mathcal{G}_{\bar{\eta}}$ be the induced isomorphism. Then the diagram

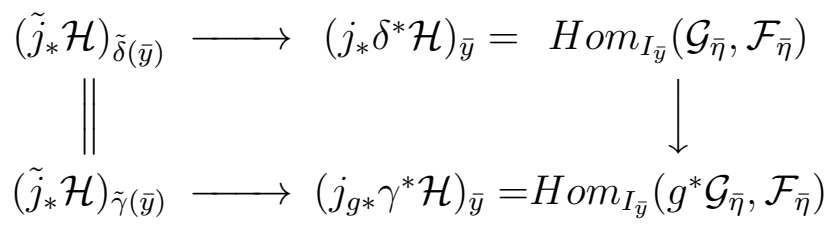

is commutative, where the horizontal arrows are the isomorphisms (3.3) and the right vertical arrow is induced by the isomorphism $\bar{g}^{*}: g^{*} \mathcal{G}_{\bar{\eta}} \rightarrow \mathcal{G}_{\bar{\eta}}$.

Proof. 1. It is sufficient to show that the map $\left(\tilde{j}_{*} \mathcal{H}\right)_{\tilde{\gamma}(\bar{y})} \rightarrow\left(j_{g *} \gamma^{*} \mathcal{H}\right)_{\bar{y}}$ is an isomorphism for each geometric point $\bar{y}$ of $\widetilde{Y}_{g} \backslash V$. Let $\bar{\eta}$ be a geometric point of $V_{\bar{y}}=V \times_{Y} Y_{\bar{y}}$ where $Y_{\bar{y}}$ is the strict localization. Let $I_{\bar{y}}=\pi_{1}\left(V_{\bar{y}}, \bar{\eta}\right), I_{\tilde{\gamma}(\bar{y})}=\pi_{1}\left((V \times V)_{\gamma(\bar{y})}, \tilde{\gamma}(\bar{\eta})\right)$ and $I_{g(\bar{y})}=\pi_{1}\left(V_{g(\bar{y})}, g(\bar{\eta})\right)$ be the inertia groups. We regard the $I_{\bar{y}^{-}}$set $\mathcal{F}_{\bar{\eta}}$ as an $I_{\tilde{\gamma}(\bar{y})^{-}}$

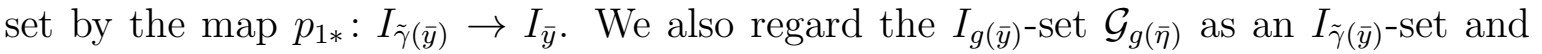
an $I_{\bar{y}}$-set by the maps $p_{2 *}: I_{\tilde{\gamma}(\bar{y})} \rightarrow I_{g(\bar{y})}$ and by $g_{*}: I_{\bar{y}} \rightarrow I_{g(\bar{y})}$. Then, the stalks $\left(\tilde{j}_{*} \mathcal{H}\right)_{\tilde{\gamma}(\bar{y})}$ and $\left(j_{g *} \gamma^{*} \mathcal{H}\right)_{\bar{y}}$ are naturally identified with the sets $\operatorname{Hom}_{I_{\tilde{\gamma}(\bar{y})}}\left(\mathcal{G}_{g(\bar{\eta})}, \mathcal{F}_{\bar{\eta}}\right)$ and $\operatorname{Hom}_{I_{\bar{y}}}\left(\mathcal{G}_{g(\bar{\eta})}, \mathcal{F}_{\bar{\eta}}\right)$ respectively. Since $p_{1 *} \circ \tilde{\gamma}_{*}=$ id and $p_{2 *} \circ \tilde{\gamma}_{*}=g_{*}$, it suffices to show that the images of $I_{\bar{y}}$ and of $I_{\tilde{\gamma}(\bar{y})}$ by the compositions

$$
I_{\bar{y}} \stackrel{\tilde{\gamma}_{*}}{\longrightarrow} I_{\tilde{\gamma}(\bar{y})} \stackrel{\left(p_{1 *}, p_{2 *}\right)}{\longrightarrow} I_{\bar{y}} \times I_{g(\bar{y})} \longrightarrow \operatorname{Aut}\left(\mathcal{F}_{\bar{\eta}}\right) \times \operatorname{Aut}\left(\mathcal{G}_{g(\bar{\eta})}\right)
$$

are the same.

Take a basis $t_{i}$ of $O\left(-D_{i}\right)$ for each $i \in I$ at $\bar{y}$ and an integer $m$ invertible at $y$ such that the image of $I_{\bar{y}} \rightarrow \operatorname{Aut}\left(\mathcal{F}_{\bar{\eta}}\right)$ is a quotient of the image of the map $t: I_{\bar{y}} \rightarrow M=$ $\prod_{i \in I} \mu_{m}$ (3.1). We also take a basis $s_{i}$ of $O\left(-D_{i}\right)$ for each $i$ at $g(y)$ such that the image of $I_{g(\bar{y})} \rightarrow \operatorname{Aut}\left(\mathcal{G}_{g(\bar{\eta})}\right)$ is a quotient of the image of $s: I_{g(\bar{y})} \rightarrow M=\prod_{i \in I} \mu_{m}$. Since $\bar{y}$ is a geometric point of $(Y \times Y)^{\sim}$, there exists a unit $u_{i}$ on a neighborhood of $\bar{y}$ such that $g^{*}\left(s_{i}\right)=u_{i} t_{i}$ for each $i \in I$. Since the $m$-th roots of $u_{i}$ define an étale covering, 
we have a commutative diagram

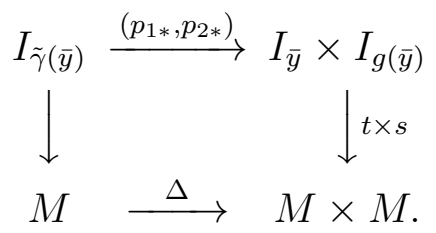

Since the map $\tilde{\gamma}_{*}: I_{\bar{y}} \rightarrow I_{\tilde{\gamma}(\bar{y})}$ is a section of $p_{1 *}: I_{\tilde{\gamma}(\bar{y})} \rightarrow I_{\bar{y}}$, both $\operatorname{Image}\left(I_{\bar{y}} \rightarrow M \times M\right)$ and Image $\left(I_{\tilde{\gamma}(\bar{y})} \rightarrow M \times M\right)$ are equal to $\Delta\left(\right.$ Image $\left.t: I_{\bar{y}} \rightarrow M\right)$. Thus the assertion is proved.

2. The assertion is étale local. We may assume there exists a basis $t_{i}$ of $O\left(-D_{i}\right)$ for each $i \in I$ and $\mathcal{G}$ is trivialized by the étale covering $W=V\left[T_{i}, i \in I\right] /\left(T_{i}^{m}-t_{i}, i \in I\right) \rightarrow$ $V$. Thus, it is reduced to the case where $\mathcal{G}$ is the locally constant sheaf represented by the étale covering $W \rightarrow V$.

Let $\tilde{u}_{i} \in \Gamma\left((Y \times Y)^{\sim}, O^{\times}\right)$be the unit defined by the equation $1 \otimes t_{i}=\tilde{u}_{i}\left(t_{i} \otimes 1\right)$. Since $\tilde{\gamma}(y)=\tilde{\delta}(y)$, we have $u_{i}(y)=\tilde{u}_{i}(\tilde{\gamma}(y))=\tilde{u}_{i}(\tilde{\delta}(y))=1$. Hence there exists a unique unit $\tilde{v}_{i} \in \Gamma\left((Y \times Y)_{\bar{y}}, O^{\times}\right)$on the strict localization such that $\tilde{v}_{i}^{m}=\tilde{u}_{i}$ and $\tilde{v}_{i}(\bar{y})=1$. Then, by sending $1 \otimes T_{i}$ to $\tilde{v}_{i}\left(T_{i} \otimes 1\right)$, we obtain an isomorphism $p_{2}^{*} \mathcal{G} \rightarrow p_{1}^{*} \mathcal{G}$. The pull-back of this isomorphism by $\gamma$ defines an isomorphism $g^{*} \mathcal{G} \rightarrow \mathcal{G}$. The diagram (3.4) is commutative if we define the right vertical arrow to be that induced by this isomorphism $g^{*} \mathcal{G} \rightarrow \mathcal{G}$.

On the other hand, we have $u_{i}=\tilde{\gamma}^{*}\left(\tilde{u}_{i}\right)$ and hence $v_{i}=\tilde{\gamma}^{*}\left(\tilde{v}_{i}\right)$ for each $i \in I$. By the assumption $\bar{g}^{*}\left(\sqrt[m]{t_{i}}\right)=v_{i} \sqrt[m]{t_{i}}$ for each $i \in I$, the map $\bar{g}^{*}: g^{*} \mathcal{G}_{\bar{\eta}} \rightarrow \mathcal{G}_{\bar{\eta}}$ is also induced by the isomorphism $g^{*} \mathcal{G} \rightarrow \mathcal{G}$ defined above. Thus the assertion follows.

We apply Proposition 3.1.4 to the following situation. We consider a cartesian diagram

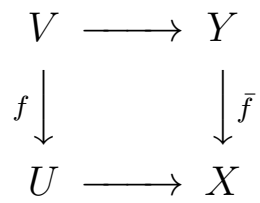

of normal schemes over a perfect field $k$ satisfying the following conditions. The horizontal arrows are open immersions with dense images. The map $f: V \rightarrow U$ is a finite étale Galois covering of Galois group $G$. The map $\bar{f}: Y \rightarrow X$ is proper and the action of $G$ on $V$ is extended to an action on $Y$ over $X$.

Let $D_{i} \subset Y, i \in I$ be a finite family of Cartier divisors of $Y$ such that $V \cap D_{i}=\emptyset$ for each $i \in I$. Let $\mathcal{F}$ be a smooth $E$-sheaf on $U$ and put $\mathcal{F}_{V}=f^{*} \mathcal{F}$ on $V$. We assume that $\mathcal{F}_{V}$ is of Kummer type with respect to $D_{i}, i \in I$. We put $\mathcal{H}=\mathcal{H} o m\left(\operatorname{pr}_{2}^{*} \mathcal{F}_{V}, \operatorname{pr}_{1}^{*} \mathcal{F}_{V}\right)$ on $V \times V$.

For $\sigma \in G$, applying the construction of the diagram (3.2) to the map $\sigma: V \rightarrow V$, we obtain an open immersion $j_{\sigma}: V \rightarrow \widetilde{Y}_{\sigma}$ and an immersion $\tilde{\gamma}_{\sigma}: \tilde{Y}_{\sigma} \rightarrow(Y \times Y)^{\sim}$. We identify $\delta^{*} \mathcal{H}=\mathcal{E} n d\left(\mathcal{F}_{V}\right)$ and $\gamma^{*} \mathcal{H}=\mathcal{H o m}\left(\sigma^{*} \mathcal{F}_{V}, \mathcal{F}_{V}\right)$. Let $y \in \widetilde{Y}_{\sigma} \backslash V$ be a point satisfying $\tilde{\gamma}_{\sigma}(y)=\tilde{\delta}(y)$ and $\bar{y}$ be a geometric point above $y$. The base change maps 
induce isomorphisms

$$
\left(j_{\sigma *} \mathcal{H o m}\left(\sigma^{*} \mathcal{F}_{V}, \mathcal{F}_{V}\right)\right)_{\bar{y}} \longleftarrow \tilde{j}_{*} \mathcal{H}_{\bar{y}} \longrightarrow\left(j_{*} \mathcal{E} n d\left(\mathcal{F}_{V}\right)\right)_{\bar{y}}
$$

by Proposition 3.1 .4 . 1.

We recall the definition of the Brauer trace. Recall that $F$ is the residue field of an $\ell$-adic field $E$. For an automorphism $a$ of an $F$-vector space $M$ of dimension $n$, the Brauer trace $\operatorname{Tr}^{B r}(a: M) \in E$ is defined as follows. Let $\operatorname{det}(T-a: M)=$ $\left(T-\alpha_{1}\right) \cdots\left(T-\alpha_{n}\right) \in F[T]$ be the eigenpolynomial of $a$ and let $\tilde{\alpha}_{1}, \ldots, \tilde{\alpha}_{n} \in E^{\mathrm{ur}}$ be the Teichmüller liftings of $\alpha_{1}, \ldots, \alpha_{n}$. Then the Brauer trace $\operatorname{Tr}^{B r}(a: M)$ is defined to be the sum $\tilde{\alpha}_{1}+\cdots+\tilde{\alpha}_{n}$.

Corollary 3.1.5 Let the notation be as above. Assume that the pull-back $\mathcal{F}_{V}=f^{*} \mathcal{F}$ is of Kummer type with respect to $D_{i}, i \in I$. Assume further that the reduction $\overline{\mathcal{F}}_{V}$ $\bmod \lambda$ is constant and let $\bar{M}$ denote the corresponding $F$-representation of $G$.

Let $\sigma \in G$ be an element of order prime to $\ell$. We regard the canonical map $\sigma^{*}: \sigma^{*} \mathcal{F}_{V}=\sigma^{*} f^{*} \mathcal{F} \rightarrow(f \circ \sigma)^{*} \mathcal{F}=f^{*} \mathcal{F}=\mathcal{F}_{V}$ as a section of $j_{\sigma *} \mathcal{H o m}\left(\sigma^{*} \mathcal{F}_{V}, \mathcal{F}_{V}\right)$. Let $y \in \widetilde{Y}_{\sigma} \backslash V$ be a point such that $\tilde{\gamma}_{\sigma}(y)=\tilde{\delta}(y)$ and $\bar{y}$ be a geometric point above $y$.

Let $\widetilde{\operatorname{Tr}}_{\bar{y}}:\left(j_{\sigma *} \mathcal{H} \operatorname{om}\left(\sigma^{*} \mathcal{F}_{V}, \mathcal{F}_{V}\right)\right)_{\bar{y}} \rightarrow E$ be the composition of

$$
\left(j_{\sigma *} \mathcal{H o m}\left(\sigma^{*} \mathcal{F}_{V}, \mathcal{F}_{V}\right)\right)_{\bar{y}} \stackrel{\text { 3.6 }}{\longrightarrow}\left(j_{*} \mathcal{E} n d\left(\mathcal{F}_{V}\right)\right)_{\bar{y}} \stackrel{\operatorname{Tr}}{\longrightarrow} E .
$$

Then, we have

$$
\widetilde{\operatorname{Tr}}_{\bar{y}}\left(\sigma^{*}\right)=\operatorname{Tr}^{B r}(\sigma: \bar{M})
$$

Proof. We put $\bar{x}=\bar{f}(\bar{y})$ and take a geometric point $\bar{\eta}$ of $V_{\bar{y}}$. Let $I_{\bar{x}}=\pi_{1}\left(U_{\bar{x}}, \bar{\eta}\right)$ be the inertia group. Then, the element $\sigma \in G$ is in the image of the natural map $I_{\bar{x}} \rightarrow G$. By the assumption that the order of $\sigma$ is prime to $\ell$, we may take an inverse image $\bar{\sigma} \in I_{\bar{x}}$ such that the pro-order of $\bar{\sigma}$ is prime to $\ell$. The map $\bar{\sigma}: \bar{\eta} \rightarrow \bar{\eta}$ is compatible with $\sigma: V \rightarrow V$.

We take an basis $t_{i}$ of $O\left(-D_{i}\right)$ for each $i \in I$. Since $\overline{\mathcal{F}}_{V}$ is constant, the image of $\pi_{1}(V, \bar{\eta}) \rightarrow \operatorname{Aut}\left(\mathcal{F}_{V, n, \bar{\eta}}\right)$ is of order a power of $\ell$ for each $n \geq 0$. Thus, in the condition (Kum) for $\mathcal{F}_{n}$, we may take a power of $\ell$ as an integer $m$. By the assumption $\tilde{\delta}(y)=\tilde{\gamma}_{\sigma}(y)$, we have $\sigma\left(t_{i}\right) / t_{i}=1$ at $y$. Since $\bar{\sigma}$ is of pro-order prime to $\ell$, for each $i \in I$ and a power $m$ of $\ell$, we have $\bar{\sigma}\left(\sqrt[m]{t_{i}}\right) / \sqrt[m]{t_{i}}=1$ at $\bar{y}$. Thus by Proposition 3.1.4 2, the composition $\left(j_{\sigma *} \mathcal{H} \text { om }\left(\sigma^{*} \mathcal{F}_{V}, \mathcal{F}_{V}\right)\right)_{\bar{y}} \rightarrow\left(j_{*} \mathcal{E} n d\left(\mathcal{F}_{V}\right)\right)_{\bar{y}}$ is induced by the map $\bar{\sigma}^{*}: \sigma^{*} \mathcal{F}_{V, \bar{\eta}} \rightarrow \mathcal{F}_{V, \bar{\eta}}$. Hence $\widetilde{\operatorname{Tr}}_{\bar{y}}\left(\sigma^{*}\right)$ is equal to $\operatorname{Tr}\left(\bar{\sigma}^{*}: \mathcal{F}_{\bar{\eta}}\right)$. Since the pro-order of $\bar{\sigma}$ is prime to $\ell$, the action of $\bar{\sigma}^{*}$ on $\mathcal{F}_{\bar{\eta}}$ is of finite order prime to $\ell$. Thus, we have $\operatorname{Tr}\left(\bar{\sigma}^{*}: \mathcal{F}_{\bar{\eta}}\right)=\operatorname{Tr}^{B r}(\sigma: \bar{M})$. 


\subsection{Review on the Swan class}

We briefly recall the definition of the Swan class [16. First, we recall the definition of the Swan character class. Assume $k$ is perfect and we consider a cartesian diagram

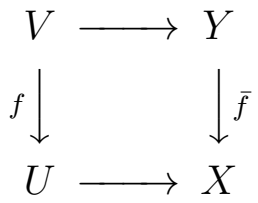

of schemes over $k$. We assume that the horizontal arrows are open immersion, that $\bar{f}: Y \rightarrow X$ is proper, and $f: U \rightarrow V$ is a finite étale Galois covering of smooth schemes of dimension $d$. Let $G$ be the Galois group $\operatorname{Gal}(V / U)$. By alteration [4] (cf. 16] Lemma 3.2.1), one can construct a diagram

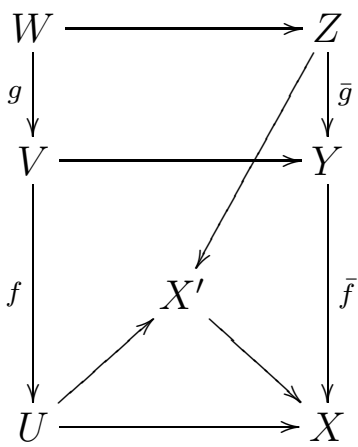

of schemes over $k$ satisfying the following properties.

(3.8.1) $U$ is the complement of a Cartier divisor $B$ of $X^{\prime}$ and the map $X^{\prime} \rightarrow X$ is an isomorphism on $U$.

(3.8,2) $Z$ is smooth over $k$ and $W$ is the complement of a divisor with simple normal crossings.

(3.8,3) The map $\bar{g}: Z \rightarrow Y$ is proper, surjective and generically finite. The squares are cartesian.

We define the log blow-up $(Z \times Z)^{\prime}$, its open subscheme $(Z \times Z)^{\sim}=(W \times W)^{\prime}$ and the $\log$ diagonal map $Z=\Delta_{Z}^{\log } \rightarrow(Z \times Z)^{\sim}=(W \times W)^{\prime}$ as in $\S 2.2$.

Let $\left(X^{\prime} \times X^{\prime}\right)^{\prime}$ be the blow-up of $X^{\prime} \times X^{\prime}$ at $B \times B$ and $p_{1}, p_{2}:\left(X^{\prime} \times X^{\prime}\right)^{\prime} \rightarrow X^{\prime}$ be the projections. Let $\left(X^{\prime} \times X^{\prime}\right)^{\sim}$ be the maximum open subscheme of $\left(X^{\prime} \times X^{\prime}\right)^{\prime}$ where $p_{1}^{*} B=p_{2}^{*} B$. Let $\left(Z \times_{X^{\prime}} Z\right)^{\sim}$ be the inverse image of the diagonal $X^{\prime} \rightarrow\left(X^{\prime} \times X^{\prime}\right)^{\sim}$ by the map $(Z \times Z)^{\sim} \rightarrow\left(X^{\prime} \times X^{\prime}\right)^{\sim}$.

For an element $\sigma \in G$, let $\Gamma_{\sigma}$ denote the graph of $\sigma$. We consider the Gysin map $(g \times g)^{!}: C H_{d}\left(V \times_{U} V \backslash \Delta_{V}\right)=\bigoplus_{\sigma \neq 1, \sigma \in G} \mathbb{Z} \cdot\left[\Gamma_{\sigma}\right] \rightarrow C H_{d}\left(W \times_{U} W \backslash W \times_{V} W\right)$ in the intersection theory [10]. For $\sigma \neq 1$, the Swan character class $s_{V / U}(\sigma) \in C H_{0}(Y \backslash V)_{\mathbb{Q}}$ is defined as the intersection product

$$
s_{V / U}(\sigma)=-\frac{1}{[W: V]} \bar{g}_{*}\left(\Gamma, \Delta_{Z}^{\log }\right)_{(Z \times Z)^{\sim}}
$$


by taking a lifting $\Gamma \in C H_{d}\left(\left(Z \times_{X^{\prime}} Z\right)^{\sim} \backslash W \times_{V} W\right)$ of $(g \times g)^{!} \Gamma_{\sigma} \in C H_{d}\left(W \times_{U} W \backslash W \times_{V}\right.$ $W)$. For $\sigma=1, s_{V / U}(\sigma) \in C H_{0}(Y \backslash V)_{\mathbb{Q}}$ is defined by requiring $\sum_{\sigma \in G} s_{V / U}(\sigma)=0$.

The following basic facts are proved in [16].

Lemma 3.2.1 1. (16] Proposition 3.2.2) The 0-cycle class $s_{V / U}(\sigma) \in C H_{0}(Y \backslash V)_{\mathbb{Q}}$ is independent of the choice of $Z, W$ and $X^{\prime}$.

Let $h: Y^{\prime} \rightarrow Y$ be a proper birational morphism inducing the identity on $V$. Then, we have $h_{*} s_{V / U}(\sigma)=s_{V / U}(\sigma)$.

2. (16] Lemma 1.1.3, 3.3.2) Assume the order of $\sigma$ is not a power of $p$ and let $\bar{C} \subset\left(Z \times_{X^{\prime}} Z\right)^{\sim}$ be the closure of the inverse image $C=(g \times g)^{-1}\left(\Gamma_{\sigma}\right)$ of the graph $\Gamma_{\sigma} \subset V \times_{U} V$ of $\sigma$. Then, there exists a diagram (3.8) such that the intersection $\bar{C} \cap \Delta_{Z}^{\log }$ is empty and hence $s_{V / U}(\sigma)=0$.

We consider an open immersion $U \rightarrow X$ of schemes over $k$. We assume $U$ is smooth of dimension $d$. For a smooth $E$-sheaf $\mathcal{F}$ on $U$, the naive Swan class is defined as follows. We take a finite étale Galois covering $V \rightarrow U$ of Galois group $G$, trivializing the reduction $\overline{\mathcal{F}}$ modulo $\lambda$. Let $\bar{M}$ be the $F$-representation of $G$ corresponding to $\overline{\mathcal{F}}$. We take a cartesian diagram (3.7) such that $\bar{f}: Y \rightarrow X$ is proper. Let $G_{(p)}$ denote the subset of $G$ consisting of elements of order a power of $p$. Then, the naive Swan class $\operatorname{Sw}^{\text {naive }}(\mathcal{F}) \in C H_{0}(X \backslash U)_{E}$ is defined by

$$
\operatorname{Sw}^{\text {naive }}(\mathcal{F})=\frac{1}{|G|} \sum_{\sigma \in G_{(p)}} \bar{f}_{*} s_{V / U}(\sigma) \operatorname{Tr}^{B r}(\sigma: \bar{M})
$$

where $\operatorname{Tr}^{B r}$ denotes the Brauer trace. The naive Swan class $\operatorname{Sw}^{\text {naive }}(\mathcal{F})$ lies in fact in $C H_{0}(X \backslash U)_{E_{0}}$ where $E_{0}=E \cap \mathbb{Q}\left(\zeta_{p^{\infty}}\right)$. The Swan class $\operatorname{Sw}(\mathcal{F}) \in C H_{0}(X \backslash U)_{\mathbb{Q}}$ is defined to be $\frac{1}{\left[E_{0}: \mathbb{Q}\right]} \operatorname{Tr}_{E_{0} / \mathbb{Q}} \mathrm{Sw}^{\text {naive }}(\mathcal{F})$. They are conjectured to be the same and known to be equal if $\operatorname{dim} X \leq 2$ [16] Lemma 4.2.3.2. It is also proved in the proof of Theorem 4.3.14 in loc. cit. that $\operatorname{deg} \operatorname{Sw}(\mathcal{F})=\operatorname{deg} \operatorname{Sw}^{\text {naive }}(\mathcal{F})$, if $X$ is proper.

\subsection{The characteristic class and the Swan class}

Now, we state and prove the main result of this section.

Theorem 3.3.1 Let $X$ be a scheme over a perfect field $k$ and $j: U \rightarrow X$ be an open immersion. We assume $U$ is smooth of dimension d. Let $\mathcal{F}$ be a smooth E-sheaf on $U$.

1. Let $f: V \rightarrow U$ be a finite étale Galois covering trivializing the reduction $\overline{\mathcal{F}}$ mod $\lambda$. Let

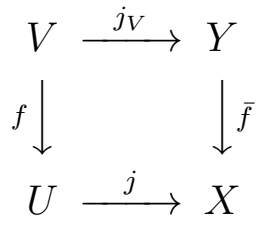


be a cartesian diagram of schemes over $k$ where $\bar{f}: Y \rightarrow X$ is proper. Let $G=$ $\operatorname{Gal}(V / U)$ be the Galois group and $\bar{M}$ be the $F$-representation of $G$ corresponding to $\overline{\mathcal{F}}$. Then, if $\mathcal{F}_{V}=f^{*} \mathcal{F}$ is of Kummer type with respect to $Y$, we have

$$
C\left(j_{V !} \mathcal{F}_{V}, \overline{\Gamma_{\sigma}}, \sigma^{*}\right)=-s_{V / U}(\sigma) \operatorname{Tr}^{B r}(\sigma: \bar{M})
$$

for $\sigma \in G, \neq 1$. If the order of $\sigma$ is not a power of $p$, the both sides are 0 .

2. Assume $\mathcal{F}$ is potentially of Kummer type with respect to $X$. Then, we have

$$
C\left(j_{!} \mathcal{F}\right)=\operatorname{rank} \mathcal{F} \cdot C\left(j_{!} E\right)-\mathrm{Sw}^{\text {naive }} \mathcal{F}
$$

in $H^{0}\left(X, \mathcal{K}_{X}\right)$.

Proof. 1. First, we show that we may assume the additional conditions $(3.9,1)$ (3.9.5) below are satisfied. By Proposition 2.1.6 and Lemma 3.2.1, we may replace $X$ and $Y$ by proper modifications. By replacing $X$ by a blow-up, we may assume that $U$ is the complement of a Cartier divisor $B$. By replacing $Y$ by the closure of the image of the immersion $(\sigma)_{\sigma \in G}: V \rightarrow\left(\prod_{\sigma \in G}\right)_{X} Y$, we may assume the action of $G$ on $V$ is extended to an action on $Y$. Let $D_{i}, i \in I$ be a finite family of Cartier divisors of $Y$ with respect to which $\mathcal{F}_{V}$ is of Kummer type. By replacing it by the family $\sigma\left(D_{i}\right),(i, \sigma) \in I \times G$, we may assume the family $D_{i}, i \in I$ is stable under the $G$-action. Further, by replacing $Y$ by the blow-up by the intersections $D_{i} \cap D_{j}$, we may assume that $O\left(-D_{i}\right)+O\left(-D_{j}\right)$ is an invertible ideal for each $i, j \in I$. Replacing $X$ and $Y$ by the normalizations, we may assume $X$ and $Y$ are normal. Thus we may assume the following conditions are satisfied.

[3.9.1) $U \subset X$ is the complement of a Cartier divisor $B$.

(3.9,2) The action of $G$ on $V$ over $U$ is extended to an action on $Y$ over $X$.

[3.9.3) $\mathcal{F}_{V}$ is of Kummer type with respect to a family $D_{i}, i \in I$ of Cartier divisors and the family $D_{i}, i \in I$ is stable under the $G$-action.

(3.9, 4) $X$ and $Y$ are normal.

(3.9.5) $O\left(-D_{i}\right)+O\left(-\sigma^{*} D_{i}\right)$ is an invertible ideal for each $i \in I$ and $\sigma \in G$.

Let $\overline{(Y \times Y)}^{\prime}$ be the normalization of the blow-up of $Y \times Y$ at $D_{i} \times D_{i}, i \in I$ as in $\S 3.1$. Let $(Y \times Y)^{\sim} \subset \overline{(Y \times Y)}^{\prime}$ be the maximum open subscheme where $p_{1}^{*} D_{i}=p_{2}^{*} D_{i}$ for each $i \in I$ and $\tilde{j}_{V}: V \times V \rightarrow(Y \times Y)^{\sim}$ be the open immersion. The closed immersion $\gamma=(1, \sigma): Y \rightarrow Y \times Y$ is uniquely lifted to a closed immersion $\tilde{\gamma}: Y \rightarrow \overline{(Y \times Y)}^{\prime}$, by the condition (3.9.5), by the universality of blow-up and the by the assumption that $Y$ is normal. The intersection $\widetilde{Y}_{\sigma}=Y \cap(Y \times Y)^{\sim}$ is the maximum open subscheme where $D_{i}=\sigma^{*} D_{i}$ for each $i \in I$ and $j_{\sigma}: V \rightarrow \widetilde{Y}_{\sigma}$ be the open immersion. 
We take an alteration

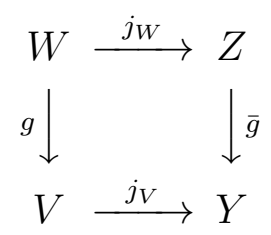

satisfying the following properties.

(3.12,1) The diagram is cartesian.

[3.12,2) The map $g: Z \rightarrow Y$ is proper, surjective and generically finite.

(3.12,3) $Z$ is smooth over $k$ and $W$ is the complement of a divisor $E=\bigcup_{j \in J} E_{j}$ with simple normal crossings.

We consider the smooth $E$-sheaf $\mathcal{F}_{W}=(f \circ g)^{*} \mathcal{F}$ on $W$, the closed subscheme $C=(g \times g)^{-1}\left(\Gamma_{\sigma}\right) \subset W \times W$ and the pull-back of the map $\sigma^{*}: \sigma^{*} \mathcal{F}_{V} \rightarrow \mathcal{F}_{V}$ on $C$. We show that they satisfy the assumptions in Proposition 2.2.3 Let $(Z \times Z)^{\sim} \subset(Z \times Z)^{\prime}$ be the $\log$ blow-up as in $\S 2.2$. Since $\overline{\mathcal{F}}$ is constant on $V$, the sheaf $\mathcal{F}_{W}$ is tamely ramified along $Z \backslash W$. By Lemma 2.2.2, the subscheme $W \times_{U} W \subset W \times W$ is non-expanding with respect to $Z$. Hence its closed subscheme $C$ is also non-expanding with respect to $Z$. Let $\widetilde{C}$ denote the closure of $C$ in $(Z \times Z)^{\sim}$. We put $\mathcal{H}=\mathcal{H o m}\left(\operatorname{pr}_{2}^{*} \mathcal{F}_{V}, \operatorname{pr}_{1}^{*} \mathcal{F}_{V}\right)$ on $V \times V$. We consider the closed immersions $\gamma: V \rightarrow V \times V$ and $\tilde{\gamma}: \widetilde{Y}_{\sigma} \rightarrow(Y \times Y)^{\sim}$. By Proposition 3.1.4 and by the assumption that $\mathcal{F}_{V}$ is of Kummer type, the restriction map $\Gamma\left(\widetilde{Y}_{\sigma},\left.\left(\tilde{j}_{V_{*}} \mathcal{H}\right)\right|_{\tilde{Y}_{\sigma}}\right) \rightarrow \Gamma\left(V, \gamma^{*} \mathcal{H}\right)$ is an isomorphism. By the commutative diagram

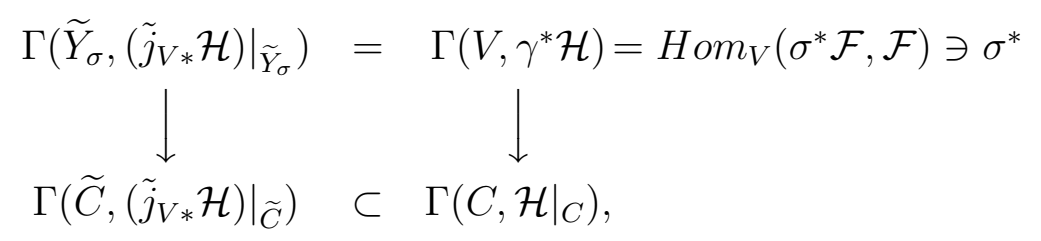

the image of $\sigma^{*}$ in $\Gamma\left(C,\left.\mathcal{H}\right|_{C}\right)$ lies in the tame part $\Gamma\left(\widetilde{C},\left.\left(\tilde{j}_{V *} \mathcal{H}\right)\right|_{\tilde{C}}\right)$. Thus the reductions $\mathcal{F}_{n}$ satisfy the assumptions in Proposition 2.2.3. By applying it to $\mathcal{F}_{n}$ and taking the limit, we obtain

$$
C\left(j_{W !} ! \mathcal{F}_{W}, \Gamma_{\sigma}, \sigma^{*}\right)=\operatorname{cl}\left((g \times g)^{!} \Gamma_{\sigma}, \Delta_{W}^{\log }\right)_{(W \times W)^{\prime}} \cdot \widetilde{T r} \delta^{\prime *} \tilde{\sigma}
$$

for $\sigma \in G$.

If the order of $\sigma$ is not a power of $p$, the intersection $\widetilde{C} \cap \Delta_{Z}^{\log }$ is empty and hence the both sides in (3.10) are 0 by Lemma 3.2.1 2. If the order of $\sigma$ is a power of $p$, we have $\widetilde{\operatorname{Tr}} \delta^{\prime *} \tilde{\sigma}=\operatorname{Tr}^{B r}(\sigma: \bar{M})$ by Corollary 3.1.5 since $\mathcal{F}_{V}$ is assumed of Kummer type. Thus (3.10) follows from the definition of the Swan character class. 
2. We take a commutative diagram (3.9) as in 1 such that the pull-back $\mathcal{F}_{V}$ is of Kummer type with respect to $Y$. Applying Lemma 1.2.5 to the reductions $\mathcal{F}_{n}$ and taking the limit, we obtain

$$
f^{*}\left(C\left(j_{!} \mathcal{F}\right)-\operatorname{rank} \mathcal{F} \cdot C\left(j_{!} E\right)\right)=\sum_{\sigma \in G}\left(C\left(j_{V !} \mathcal{F}_{V}, \Gamma_{\sigma}, \sigma^{*}\right)-\operatorname{rank} \mathcal{F} \cdot C\left(j_{V !} E, \Gamma_{\sigma}, 1\right)\right) .
$$

By 1, the right hand side is equal to

$$
-\sum_{\sigma \in G_{(p)}, \neq 1} s_{V / U}(\sigma) \cdot\left(\operatorname{Tr}^{B r}(\sigma: \bar{M})-\operatorname{rank} \mathcal{F}\right)=-\sum_{\sigma \in G_{(p)}} s_{V / U}(\sigma) \cdot \operatorname{Tr}^{B r}(\sigma: \bar{M}) .
$$

Thus, taking $f_{*}$, we obtain

$$
\begin{aligned}
|G|\left(C\left(j_{!} \mathcal{F}\right)-\operatorname{rank} \mathcal{F} \cdot C\left(j_{!} E\right)\right) & =-\sum_{\sigma \in G_{(p)}} f_{*} s_{V / U}(\sigma) \cdot \operatorname{Tr}^{B r}(\sigma: \bar{M}) \\
& =-|G| \mathrm{Sw}^{\text {naive }} \mathcal{F} .
\end{aligned}
$$

We recover the following main result of [16].

Corollary 3.3.2 (16] Theorem 4.2.9) For a smooth E-sheaf $\mathcal{F}$ on $U$, we have

$$
\chi_{c}(U, \mathcal{F})=\operatorname{rank} \mathcal{F} \cdot \chi_{c}(U, E)-\operatorname{deg} \operatorname{Sw} \mathcal{F} .
$$

Proof. Since $\operatorname{deg} \mathrm{Sw} \mathcal{F}=\operatorname{deg} \mathrm{Sw}^{\text {naive }} \mathcal{F}$, it suffices to show

$$
\chi_{c}(U, \overline{\mathcal{F}})=\operatorname{rank} \overline{\mathcal{F}} \cdot \chi_{c}(U, E)-\operatorname{deg} \operatorname{Sw}^{\text {naive }} \overline{\mathcal{F}}
$$

for a smooth $F$-sheaf $\overline{\mathcal{F}}$ on $U$. By Brauer induction, we may assume the rank of $\overline{\mathcal{F}}$ is 1 . Let $\bar{\chi}: \pi_{1}(U)^{\mathrm{ab}} \rightarrow F^{\times}$be the character corresponding to $\overline{\mathcal{F}}$ and let $\chi: \pi_{1}(U)^{\mathrm{ab}} \rightarrow E^{\times}$ be its Teichmüller lifting. Then, the order of $\chi$ is finite and the corresponding smooth $E$-sheaf $\mathcal{F}_{\chi}$ on $U$ is potentially of Kummer type. Thus, it suffices to take the trace of (3.11).

\section{Characteristic class of a sheaf of rank 1}

In this section we assume $\Lambda$ is a finite local $\mathbb{Z}_{\ell^{-}}$-algebra. We fix an inclusion $\mu_{p^{\infty}}(\Lambda) \rightarrow$ $\mathbb{Z}\left[\frac{1}{p}\right] / \mathbb{Z}$ and identify the $p$-primary part $\mu_{p \infty}(\Lambda)$ as a subgroup of $\mathbb{Z}\left[\frac{1}{p}\right] / \mathbb{Z}$. Let $X$ be a smooth scheme over $k$ and $j: U \rightarrow X$ be the open immersion of the complement of a divisor $D$ with simple normal crossings. For a smooth rank 1 sheaf $\mathcal{F}$ on $U$, we will show that the difference $C\left(j_{!} \mathcal{F}\right)-\operatorname{rank} \mathcal{F} \cdot C\left(j_{!} \Lambda\right)$ is given by the 0 -cycle class $c_{\mathcal{F}}$ defined by Kato in [15], assuming $\mathcal{F}$ is clean with respect to $D$. The definition of the 0 -cycle class $c_{\mathcal{F}}$ will be recalled in Definition 4.2.1,2. As a byproduct, we obtain a new proof of the Grothendieck-Ogg-Shafarevich formula

$$
\chi_{c}(U, \mathcal{F})=\operatorname{rank} \mathcal{F} \cdot \chi_{c}(U, \Lambda)-\operatorname{deg} \operatorname{Sw} \mathcal{F} .
$$

for curves without using the Weil formula. 


\subsection{Review on ramification of Artin-Schreier-Witt characters}

We briefly recall the ramification theory of Artin-Schreier-Witt characters according to [14] $\S 3$ and [2] $\S 10$. Let $K$ be a complete discrete valuation field of characteristic $p>0$.

Let $F$ be the residue field of $K$. Let $\Omega_{F}=\Omega_{F / F^{p}}^{1}$ be the $F$-vector space of differential 1 -forms and put

$$
\Omega_{F}(\log )=\Omega_{F} \oplus\left(F \otimes_{\mathbb{Z}} K^{\times}\right) /\left(d a-(a \otimes a): a \in O_{K}, a \neq 0\right) .
$$

For $a \in K^{\times}$, let $d \log a \in \Omega_{F}(\log )$ denote the image of $1 \otimes a$. We have a short exact sequence

$$
0 \longrightarrow \Omega_{F} \longrightarrow \Omega_{F}(\log ) \stackrel{\text { res }}{\longrightarrow} F \longrightarrow 0
$$

of $F$-vector space where the residue map res: $\Omega_{F}(\log ) \rightarrow F$ sends $d \log a$ to ord $a$. A choice of prime element defines a splitting $F \rightarrow \Omega_{F}(\log )$ of the exact sequence.

A filtration $F_{\bullet}$ on $H^{1}(K, \mathbb{Q} / \mathbb{Z})$ is defined in 14 Definition $(2.1)$ and is recalled in $2 \S 10.4$. We have $F_{-1} H^{1}(K, \mathbb{Q} / \mathbb{Z})=0$ and $F_{0} H^{1}(K, \mathbb{Q} / \mathbb{Z})$ is the unramified part. For a character $\chi \in H^{1}(K, \mathbb{Q} / \mathbb{Z})$, the Swan conductor of $\chi$ is defined to be the smallest integer $r \geq 0$ such that $\chi \in F^{r} H^{1}(K, \mathbb{Q} / \mathbb{Z})$. For $r>0$, a canonical injection

$$
\mathrm{rsw}_{r}: G r_{r}^{F} H^{1}(K, \mathbb{Q} / \mathbb{Z}) \longrightarrow \Omega_{F}(\log ) \otimes m_{K}^{-r} / m_{K}^{-r+1}
$$

is defined in Theorem (3.2) (3) [14] and is recalled in Proposition 10.7 [2].

\subsection{Blow-up of the diagonal}

Let $X$ be a smooth scheme of dimension $d$ over a perfect field $k$ and $U \subset X$ be the complement of a divisor $D=\bigcup_{i \in I} D_{i}$ with simple normal crossings. Let $j: U \rightarrow X$ denote the open immersion and let $\mathcal{F}$ be a smooth $\Lambda$-sheaf of rank 1 on $U$.

For each irreducible component $D_{i}$ of $D$, the sheaf $\mathcal{F}$ defines a character of the absolute Galois group of the local field $K_{i}$ at the generic point. By the identification $\mu_{p^{\infty}}(\Lambda) \subset \mathbb{Z}\left[\frac{1}{p}\right] / \mathbb{Z}$ fixed at the beginning of this section, the $p$-primary part of the character $\pi_{1}(U)^{\mathrm{ab}} \rightarrow \Lambda^{\times}$corresponding to $\mathcal{F}$ defines a character $\pi_{1}(U)^{\mathrm{ab}} \rightarrow \mathbb{Z}\left[\frac{1}{p}\right] / \mathbb{Z}$. Hence applying the theory recalled in the previous subsection, we define the Swan conductor $\operatorname{Sw}_{i}(\mathcal{F}) \in \mathbb{N}$ for each $i$. We define the Swan divisor $D_{\mathcal{F}}$ to be $\sum_{i \in I} \mathrm{Sw}_{i}(\mathcal{F}) D_{i}$. We decompose $D=D_{t}+D_{w}$ into the sum of the tame part $D_{t}=\sum_{\operatorname{Sw}_{i}(\mathcal{F})=0} D_{i}$ and the wild part $D_{w}=D_{\mathcal{F} \text {,red }}=\sum_{\mathrm{Sw}_{i}(\mathcal{F})>0} D_{i}$. The refined Swan conductor $\operatorname{rsw}_{i} \mathcal{F}$ defines a non-zero section of the locally free sheaf $\left.\Omega_{X / k}^{1}(\log D)\left(D_{\mathcal{F}}\right)\right|_{D_{w}}$ at the generic point $\xi_{i}$ of each irreducible component $D_{i} \subset D_{w}$. By [14] Theorem (7.1) and Proposition (7.3), there exists a unique global section $\operatorname{rsw}^{\mathcal{F}} \in \Gamma\left(D_{w},\left.\Omega_{X / k}^{1}(\log D)\left(D_{\mathcal{F}}\right)\right|_{D_{w}}\right)$ extending $\operatorname{rsw}_{i} \mathcal{F}$ for each $D_{i} \subset D_{w}$. For a locally free sheaf $\mathcal{E}$ of finite rank on a scheme $S$, let $c_{*}(\mathcal{E})=\sum_{i} c_{i}(\mathcal{E}) \in \bigoplus_{i} C H^{i}(S \rightarrow S)$ denote the total Chern class and put $c_{*}(\mathcal{E})^{*}=$ $c_{*}\left(\mathcal{E}^{*}\right)=\sum_{i}(-1)^{i} c_{i}(\mathcal{E})$. 
Definition 4.2.1 Let $X$ be a smooth scheme of dimension d over a perfect field $k$ and $U \subset X$ be the complement of a divisor $D$ with simple normal crossings. Let $\mathcal{F}$ be a smooth $\Lambda$-sheaf on $U$ of rank 1 and $\operatorname{rsw} \mathcal{F} \in \Gamma\left(D_{w},\left.\Omega_{X / k}^{1}(\log D)\left(D_{\mathcal{F}}\right)\right|_{D_{w}}\right)$ be the refined Swan character defined on the wild part $D_{w}$.

1. We say that $\mathcal{F}$ is clean with respect to $D$ if the section $\mathrm{rsw} \mathcal{F}$ is nowhere vanishing on $D_{w}$.

2. If $\mathcal{F}$ is clean with respect to $D$, we define a 0 -cycle class $c_{\mathcal{F}} \in C H_{0}\left(D_{w}\right)$ by

$$
c_{\mathcal{F}}=\left\{c_{*}\left(\Omega_{X / k}^{1}(\log D)\right)^{*} \cap\left(1+D_{\mathcal{F}}\right)^{-1} \cap\left[D_{\mathcal{F}}\right]\right\}_{\operatorname{dim} 0} .
$$

The refined Swan character may be also regarded as an $O_{D_{w}}$-linear map rsw $\mathcal{F}: O_{D_{w}}\left(-D_{\mathcal{F}}\right) \rightarrow$ $\left.\Omega_{X / k}^{1}(\log D)\right|_{D_{w}}$. Then, we have

$$
c_{\mathcal{F}}=(-1)^{d-1} c_{d-1}\left(\operatorname{Coker}\left(\operatorname{rsw} \mathcal{F}:\left.O_{D_{w}}\left(-D_{\mathcal{F}}\right) \rightarrow \Omega_{X / k}^{1}(\log D)\right|_{D_{w}}\right)\right) \cap\left[D_{\mathcal{F}}\right]
$$

In [16], the equality $c_{\mathcal{F}}=\operatorname{Sw}(\mathcal{F})$ is proved if $d \leq 2$ in Theorem 5.1 .5 and is conjectured in general in Conjecture 5.1.1.

Let $f:(X \times X)^{\prime} \rightarrow X \times X$ be the log blow-up with respect to $D \subset X$ and let $X \rightarrow(X \times X)^{\prime}$ be the log diagonal as in $\S 2.2$. Recall that $(U \times X)^{\prime},(X \times U)^{\prime} \subset(X \times X)^{\prime}$ are the complement of the proper transforms of $D \times X$ and of $X \times D$ respectively. In this section, we write $(U \times U)^{\prime}$ for the log product $(X \times X)^{\sim}=(U \times X)^{\prime} \cap(X \times U)^{\prime}$.

We regard $D_{\mathcal{F}} \subset X$ as a closed subscheme of $(X \times X)^{\prime}$ by the log diagonal map $X \rightarrow(X \times X)^{\prime}$ and let $\bar{g}:(X \times X)^{\prime \prime} \rightarrow(X \times X)^{\prime}$ be the blow-up at $D_{\mathcal{F}}$. Let $(U \times$ $U)^{\prime \prime},(U \times X)^{\prime \prime},(X \times U)^{\prime \prime} \subset(X \times X)^{\prime \prime}$ be the inverse image of $(U \times U)^{\prime},(U \times X)^{\prime},(X \times U)^{\prime}$ respectively. Let $\Delta_{i} \subset(X \times X)^{\prime}$ be the exceptional divisor above $D_{i} \times D_{i}$ for each $i \in I$. Let $(U \times U)^{\prime \prime \prime} \subset(U \times U)^{\prime \prime}$ be the complement of the union of the proper transforms of $\Delta_{i}$ for $D_{i} \subset D_{w}$. We consider a commutative diagram

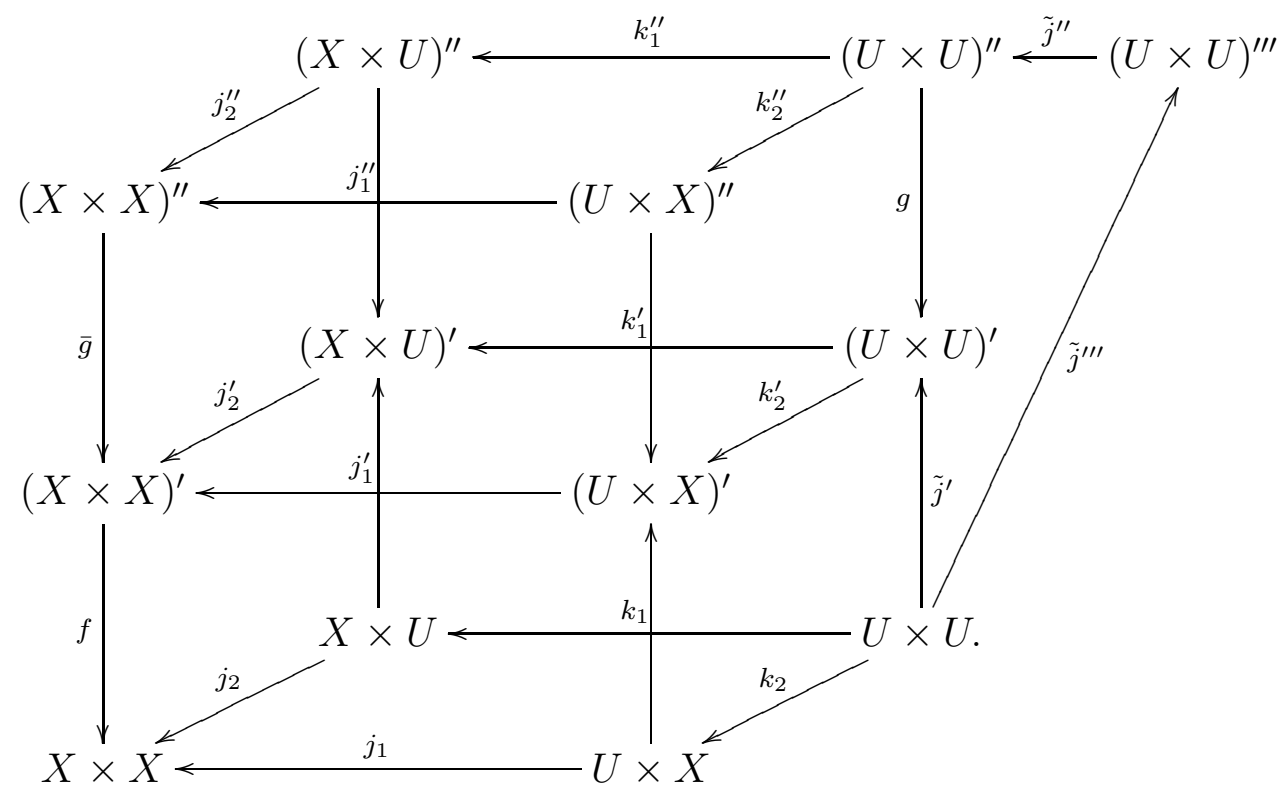


The vertical down arrows are blowing-ups and the other arrows are open immersions. The faces of the upper cube and the four faces consisting of open immersions of the lower cube are cartesian.

The projections $p_{1}, p_{2}:(U \times U)^{\prime \prime \prime} \rightarrow X$ are smooth of dimension $d$ and hence $(U \times$ $U)^{\prime \prime \prime}$ is smooth of dimension $2 d$ over $k$. The $\log$ diagonal $X \rightarrow(U \times U)^{\prime}$ is uniquely lifted to a closed immersion $X \rightarrow(U \times U)^{\prime \prime \prime}$. We identify the exceptional divisor of the blow-up $g:(U \times U)^{\prime \prime} \rightarrow(U \times U)^{\prime}$ with the $\mathbf{P}^{d}$-bundle $\mathbf{P}\left(\left.\Omega_{X / k}^{1}(\log D)\right|_{D_{w}} \oplus N_{D_{\mathcal{F}} / X}\right)=$ $\mathbf{P}\left(\left.\Omega_{X / k}^{1}(\log D)\left(D_{\mathcal{F}}\right)\right|_{D_{w}} \oplus O_{D_{w}}\right)$ over $D_{w}$. Under this identification, the inverse image $(U \times U)^{\prime \prime \prime} \times_{(U \times U)} D_{w}=(U \times U)^{\prime \prime \prime} \backslash(U \times U)$ of $D_{w}$ is identified with the $\mathbf{A}^{d}$-bundle $\mathbf{V}\left(\left.\Omega_{X / k}^{1}(\log D)\left(D_{\mathcal{F}}\right)\right|_{D_{w}}\right)$ over $D_{w}$. Here $\mathbf{P}(\mathcal{E})=\operatorname{Proj}\left(S^{\bullet} \mathcal{E}\right)$ and $\mathbf{A}(\mathcal{E})=\operatorname{Spec}\left(S^{\bullet} \mathcal{E}\right)$ for a locally free sheaf $\mathcal{E}$.

Proposition 4.2.2 Let $X$ be a smooth scheme of dimension d over a perfect field $k$ and $U \subset X$ be the complement of a divisor $D$ with simple normal crossings. Let $\mathcal{F}$ be a smooth $\Lambda$-sheaf on $U$ of rank 1 and $D_{\mathcal{F}}=\mathrm{Sw}(\mathcal{F})$ be the Swan divisor as above. We put $\mathcal{H}_{0}=\mathcal{H}$ om $\left(\operatorname{pr}_{2}^{*} \mathcal{F}, \operatorname{pr}_{1}^{*} \mathcal{F}\right)$ on $U \times U$. Then, we have the following.

1. The $\Lambda$-sheaf $\mathcal{H}_{0}^{\prime \prime \prime}=\tilde{j}_{*}^{\prime \prime \prime} \mathcal{H}_{0}$ is a smooth $\Lambda$-sheaf of rank 1 on $(U \times U)^{\prime \prime \prime}$.

2. The restriction of the smooth $\Lambda$-sheaf $\mathcal{H}_{0}^{\prime \prime \prime}$ on the complement $(U \times U)^{\prime \prime \prime} \backslash(U \times U)$ is the Artin-Schreier sheaf defined by the minus of the refined Swan character $\mathrm{rsw} \mathcal{F} \in$ $\Gamma\left(D_{w},\left.\Omega_{X / k}^{1}(\log D)\left(D_{\mathcal{F}}\right)\right|_{D_{w}}\right)$ regarded as a linear form on $(U \times U)^{\prime \prime \prime} \backslash(U \times U)=$ $\mathbf{V}\left(\left.\Omega_{X / k}^{1}(\log D)\left(D_{\mathcal{F}}\right)\right|_{D_{w}}\right)$.

First, we verify it at the generic point of each irreducible component of $(U \times U)^{\prime \prime \prime} \backslash$ $(U \times U)=\mathbf{V}\left(\left.\Omega_{X / k}^{1}(\log D)\left(D_{\mathcal{F}}\right)\right|_{D_{w}}\right)$. Let $D_{i}$ be an irreducible component of $D_{w}$ and $\xi_{i}$ be the generic point of $D_{i}$. Let $K_{i}$ be the local field of $X$ at $\xi_{i}$ and let $\chi_{i}: G_{K_{i}}^{\mathrm{ab}} \rightarrow \Lambda^{\times}$ be the character defined by the sheaf $\mathcal{F}$. The function field $F_{i}$ of $D_{i}$ is the residue field of $K_{i}$. Put $n_{i}=\mathrm{Sw}_{K_{i}} \chi_{i}>0$ and let $\mathrm{rsw}_{i} \in \Omega_{F_{i}}(\log ) \otimes m_{K_{i}}^{-n_{i}} / m_{K_{i}}^{-n_{i}+1}$ denote the refined Swan character of the $p$-primary part of $\chi_{i}$. Let $\eta_{i}$ be the generic point of the divisor $(U \times U)^{\prime \prime \prime} \times_{(U \times U)^{\prime}} D_{i}$, let $L_{i}$ be the local field at $\eta_{i}$ and $E_{i}$ be the residue field. Let $\varphi_{i}: G_{L_{i}}^{\text {ab }} \rightarrow \Lambda^{\times}$be the character defined by the sheaf $\mathcal{H}_{0}$. We have an inclusion $\Omega_{F_{i}}(\log ) \otimes m_{K_{i}}^{-n_{i}} / m_{K_{i}}^{-n_{i}+1}=\Omega_{X / k}^{1}(\log D)\left(D_{\mathcal{F}}\right)_{\xi_{i}} \subset E_{i}$.

The following lemma is a consequence of [2] Proposition 13.6.

Lemma 4.2.3 Let the notation be as above and $n_{i}=\mathrm{Sw}_{K_{i}} \chi_{i}>0$. Then we have the following.

1. The character $\varphi_{i}: G_{L_{i}}^{\mathrm{ab}} \rightarrow \Lambda^{\times}$is unramified and is of order $p$.

2. The character $\varphi_{i}$ regarded as an element of $H^{1}\left(L_{i}, \mathbb{Z} / p \mathbb{Z}\right)$ is the image of the minus of the refined Swan character $\operatorname{rsw} \chi_{i} \in \Omega_{F_{i}}(\log ) \otimes m_{\xi_{i}}^{-n_{i}} / m_{\xi_{i}}^{-n_{i}+1}$ by the canonical map

$$
\Omega_{F_{i}}(\log ) \otimes m_{\xi_{i}}^{-n_{i}} / m_{\xi_{i}}^{-n_{i}+1} \subset E_{i} \longrightarrow H^{1}\left(E_{i}, \mathbb{Z} / p \mathbb{Z}\right) \subset H^{1}\left(L_{i}, \mathbb{Z} / p \mathbb{Z}\right)
$$

of Artin-Schreier theory. 
Corollary 4.2.4 The smooth sheaf $\mathcal{H}_{0}$ on $U \times U$ is ramified along the component $\Delta_{i}$ of the exceptional divisor $(U \times U)^{\prime} \backslash(U \times U)$ over $D_{i} \times D_{i}$ if $n_{i}=\mathrm{Sw}_{K_{i}} \chi_{i}>0$.

Proof. We prove it by contradiction. Assume the sheaf $\mathcal{H}_{0}$ is unramified along $\Delta_{i}$. Then the sheaf $\mathcal{H}_{0}$ is extended to a smooth sheaf on a neighborhood of the generic point $\xi_{i}$ of $D_{i}=\Delta_{i} \cap \Delta_{X}^{\log }$. Then, the character $\varphi_{i} \in H^{1}\left(L_{i}, \mathbb{Z} / p \mathbb{Z}\right)$ is defined by the pull-back of a character in $G_{\kappa\left(\xi_{i}\right)}^{\mathrm{ab}}=G_{F_{i}}^{\mathrm{ab}} \rightarrow \Lambda^{\times}$. This contradicts to Lemma 4.2.3 2 .

Proof of Proposition 4.2.2. 1. It follows from the assertion 1 in Lemma 4.2.3 and the purity of branch locus.

2. It follows from the assertion 2 in Lemma 4.2.3.

Corollary 4.2.5 Let the notation be as in Proposition 4.2.2. We put $\mathcal{H}_{0}^{\prime}=\tilde{j}_{*}^{\prime} \mathcal{H}_{0}$. Then, we have the following.

1. The canonical map $j_{1 !} R k_{2 *} \mathcal{H}_{0}=R j_{2 *} k_{1 !} \mathcal{H}_{0} \rightarrow R f_{*} j_{1 !}^{\prime} R k_{2 *}^{\prime} \mathcal{H}_{0}^{\prime}=R f_{*} R j_{2 *}^{\prime} k_{1 !}^{\prime} \mathcal{H}_{0}^{\prime}$ is an isomorphism.

2. If $\mathcal{F}$ is clean with respect to $D$, the identity of $\mathcal{H}_{0}$ is extended to an isomorphism $\mathcal{H}_{0}^{\prime} \rightarrow R g_{*} \tilde{j}_{!}^{\prime \prime} \mathcal{H}_{0}^{\prime \prime \prime}$

Proof. 1. It suffices to show that the restrictions of $R f_{*} j_{1 !}^{\prime} R k_{2 *}^{\prime} \mathcal{H}_{0}^{\prime}$ and $R f_{*} R j_{2 *}^{\prime} k_{1 !}^{\prime} \mathcal{H}_{0}^{\prime}$ are 0 on $D \times D$. Since the assertion is étale local on $X$, we may assume the following conditions are satisfied: We have $X=X_{1} \times X_{2}, U=U_{1} \times U_{2}$ and $\mathcal{F}=\mathcal{F}_{1} \otimes \mathcal{F}_{2}$. The open subschemes $U_{1} \subset X_{1}$ and $U_{2} \subset X_{2}$ are the complement of divisors $D_{1} \subset X_{1}$ and $D_{2} \subset X_{2}$ with simple normal crossings. The rank 1 sheaf $\mathcal{F}_{1}$ on $U_{1}$ is tamely ramified along $D_{1}$ and $\mathcal{F}_{2}$ on $U_{2}$ is wildly ramified along each component of $D_{2}$.

Then, by the Künneth formula, it suffices to show the cases where $X=X_{1}$ and $X=X_{2}$ respectively. The case $\mathcal{F}$ is tamely ramified is proved in Lemma 2.2.4. Assume $\mathcal{F}$ is wildly ramified along each component of $D$. Then, by Corollary 4.2.4 the sheaf $\mathcal{H}_{0}$ is ramified along each component of the exceptional divisor $(U \times U)^{\prime} \backslash(U \times U)$. Thus we have $\mathcal{H}_{0}^{\prime}=\tilde{j}_{!}^{\prime} \mathcal{H}_{0}$ and the assertion follows.

2. Let $g_{0}: T=(U \times U)^{\prime \prime \prime} \backslash(U \times U) \rightarrow D_{w}$ denote the restriction of $g:(U \times U)^{\prime \prime} \rightarrow$ $(U \times U)^{\prime}$. It suffices to show $R g_{0 !}\left(\left.\mathcal{H}_{0}^{\prime \prime \prime}\right|_{T}\right)=0$. By Proposition 4.2.2 2, the restriction $\left.\mathcal{H}_{0}^{\prime \prime \prime}\right|_{T}$ is the Artin-Schreier sheaf defined by the refined Swan conductor rsw $\mathcal{F}$ on the $\mathbf{A}^{d}$-bundle $T=\mathbf{V}\left(\left.\Omega_{X / k}^{1}(\log D)\left(D_{\mathcal{F}}\right)\right|_{D_{w}}\right)$. By the assumption that $\operatorname{rsw}^{\mathcal{F}}$ is nowhere vanishing, the restriction of $\mathcal{H}_{0}^{\prime \prime \prime}$ on every geometric fiber is an Artin-Schreier sheaf on $\mathbf{A}^{d}$ defined by a non-trivial linear form. Thus the assertion follows.

Now, we are ready to prove the main result of this section.

Theorem 4.2.6 Let $X$ be a smooth scheme of dimension $d$ over a perfect field $k$ and $U \subset X$ be the complement of a divisor with simple normal crossings. Let $\mathcal{F}$ be a smooth $\Lambda$-sheaf on $U$ of rank 1 . If $\mathcal{F}$ is clean with respect to $D$, we have

$$
C(j ! \mathcal{F})=C(j ! \Lambda)-c_{\mathcal{F}}
$$

in $H^{2 d}(X, \Lambda(d))$. 
Proof. We keep the notation in Proposition 4.2.2. We put $\mathcal{H}_{0}=\mathcal{H}$ om $\left(\operatorname{pr}_{2}^{*} \mathcal{F}, \operatorname{pr}_{1}^{*} \mathcal{F}\right)$ and $\mathcal{H}_{0}^{*}=\mathcal{H} o m\left(\operatorname{pr}_{1}^{*} \mathcal{F}, \operatorname{pr}_{2}^{*} \mathcal{F}\right)$ on $U \times U$. By Proposition loc. cit., the sheaves $\mathcal{H}_{0}^{\prime \prime \prime}=\tilde{j}_{*}^{\prime \prime \prime} \mathcal{H}_{0}$ and $\mathcal{H}_{0}^{\prime \prime \prime *}=\tilde{j}_{*}^{\prime \prime \prime} \mathcal{H}_{0}^{*}$ on $(U \times U)^{\prime \prime \prime}$ are smooth of rank 1 . The natural pairing $\mathcal{H}_{0} \otimes \mathcal{H}_{0}^{*} \rightarrow \Lambda$ on $U \times U$ is extended uniquely to a pairing $\mathcal{H}_{0}^{\prime \prime \prime} \otimes \mathcal{H}_{0}^{\prime \prime \prime *} \rightarrow \Lambda$ on $(U \times U)^{\prime \prime \prime}$.

Further we put $\overline{\mathcal{H}}=j_{1 !} R k_{2 *} \mathcal{H}_{0}(d)[2 d]$ and $\overline{\mathcal{H}}^{*}=j_{2 !} R k_{1 *} \mathcal{H}_{0}^{*}(d)[2 d]$ on $X \times X$ and $\overline{\mathcal{H}}^{\prime \prime}=j_{1 !}^{\prime \prime} R k_{2 *}^{\prime \prime} \tilde{j}_{!}^{\prime \prime} \mathcal{H}_{0}^{\prime \prime \prime}(d)[2 d]$ and $\overline{\mathcal{H}}^{\prime \prime *}=j_{2 !}^{\prime \prime} R k_{1 *}^{\prime \prime} \tilde{j}_{!}^{\prime \prime} \mathcal{H}_{0}^{\prime \prime \prime *}(d)[2 d]$ on $(X \times X)^{\prime \prime}$. We define $\tilde{1} \in H_{X}^{0}\left((X \times X)^{\prime \prime}, \overline{\mathcal{H}}^{\prime \prime}\right)=\Gamma\left(X, j_{*} \mathcal{E} n d(\mathcal{F})\right)=\Gamma(U, \mathcal{E} n d(\mathcal{F}))$ to be the identity. We also define $\tilde{1}^{*} \in H_{X}^{0}\left((X \times X)^{\prime \prime}, \overline{\mathcal{H}}^{\prime \prime *}\right)$ to be the identity. By Corollary 4.2.5, we obtain canonical isomorphisms $\overline{\mathcal{H}} \rightarrow R(f \circ \bar{g})_{*} \overline{\mathcal{H}}^{\prime \prime}$ and $\overline{\mathcal{H}}^{*} \rightarrow R(f \circ \bar{g})_{*} \overline{\mathcal{H}}^{\prime \prime *}$. The pairing $\mathcal{H}_{0}^{\prime \prime \prime} \otimes \mathcal{H}_{0}^{\prime \prime \prime *} \rightarrow \Lambda$ on $(U \times U)^{\prime \prime \prime}$ induces a pairing $\overline{\mathcal{H}}^{\prime \prime} \otimes \overline{\mathcal{H}}^{\prime \prime *}=\left(j_{1}^{\prime} \circ k_{2}^{\prime} \circ \tilde{j}^{\prime \prime}\right)_{!}\left(\mathcal{H}_{0}^{\prime \prime \prime} \otimes\right.$ $\left.\mathcal{H}_{0}^{\prime \prime \prime *}(2 d)[4 d]\right) \rightarrow \mathcal{K}_{(X \times X)^{\prime \prime}}$ on $(X \times X)^{\prime \prime}$.

Thus the assumptions in Proposition 2.1 .12 are satisfied. Since both $(f \circ \bar{g})_{*} \tilde{1}$ and $(f \circ \bar{g})_{*} \tilde{1}^{*}$ are equal to $j_{!} 1=1$, we obtain $C\left(j_{!} \mathcal{F}\right)=\left\langle\tilde{1}, \tilde{1}^{*}\right\rangle$. By the compatibility (1.12), the right hand side is further equal to $\operatorname{cl}(X, X)_{(X \times X)^{\prime \prime}}$. Since the conormal sheaf $N_{X /(X \times X)^{\prime \prime}}$ is isomorphic to $\Omega_{X / k}(\log D)\left(D_{\mathcal{F}}\right)$, the self-intersection product $(X, X)_{(X \times X)^{\prime \prime}}$ is equal to the 0 -cycle class

$$
(-1)^{d} c_{d}\left(\Omega_{X / k}(\log D)\left(D_{\mathcal{F}}\right)\right) \cap[X]=\left\{c_{*}\left(\Omega_{X / k}(\log D)\right)\left(1-D_{\mathcal{F}}\right)^{-1} \cap[X]\right\}_{\operatorname{dim} 0}^{*} .
$$

Thus the assertion follows.

As in Section 3, let $E$ denote a finite extension of $\mathbb{Q}_{\ell}$.

Corollary 4.2.7 Let $X$ be a scheme over a perfect field $k$ and $U \subset X$ be a dense open subscheme smooth over $k$. Let $\mathcal{F}$ be a smooth $E$-sheaf of rank 1 on $U$ and let $j: U \rightarrow X$ be the open immersion. If $\operatorname{dim} U \leq 2$, we have

$$
C\left(j_{!} \mathcal{F}\right)=C\left(j_{!} E\right)-\mathrm{Sw} \mathcal{F}
$$

in $H^{0}\left(X, \mathcal{K}_{X}\right)$.

Proof. By Proposition 2.1.6 and Lemma 3.2.1, we may replace $X$ by a proper modification. Hence, by [15] Theorem 4.1, we may assume that $X$ is smooth, that $U \subset X$ is the complement of a divisor with simple normal crossings and that $\mathcal{F}$ is clean with respect to the boundary. Then the assertion follows from Theorem 4.2.6 applied to the reductions $\mathcal{F}_{n}$ and the equality $\operatorname{Sw}(\mathcal{F})=c_{\mathcal{F}}([16]$ Theorem 5.1.5).

Remark. By the argument in the proof of Corollaries 3.3.2 4.2.7 we obtain the Grothendieck-Ogg-Shafarevich formula

(3.13) $\chi_{c}\left(U_{\bar{k}}, \mathcal{F}\right)=\operatorname{rank} \mathcal{F} \cdot \chi_{c}\left(U_{\bar{k}}, \mathbb{Q}_{\ell}\right)-\operatorname{deg} \operatorname{Sw} \mathcal{F}$

for a smooth $E$-sheaf $\mathcal{F}$ if $\operatorname{dim} U \leq 2$. This proof does not use the Weil formula. 


\section{Localized characteristic class.}

Let $X$ be a smooth scheme over $k$ and $S \subset X$ be a closed subscheme such that the complement $U$ is dense. Let $\mathcal{F}$ be an object of $D_{\text {ctf }}(X)$ and assume that the cohomology sheaves $\mathcal{H}^{q} \mathcal{F}$ are smooth on $U$. In this section, we will define a localization $C_{S}^{0}(\mathcal{F}) \in H_{S}^{0}\left(X, \mathcal{K}_{X}\right)$ of the characteristic class as a cohomology class supported on $S$.

\subsection{Discrepancy of the map (1.7).}

Let $i: Z \rightarrow X$ be a closed immersion of schemes over $k$. For an object $\mathcal{F}$ of $D^{-}(Y, \Lambda)$, we have a canonical map $i^{*} \mathcal{F} \otimes^{L} R i^{!} \Lambda \rightarrow R i^{!} \mathcal{F}$ (1.7). We construct a functor

$$
\Delta_{i}: D^{-}(X, \Lambda) \rightarrow D^{-}(Z, \Lambda)
$$

fitting in a distinguished triangle

$$
\rightarrow i^{*} \mathcal{F} \otimes^{L} R i^{!} \Lambda \rightarrow R i^{!} \mathcal{F} \rightarrow \Delta_{i} \mathcal{F} \rightarrow .
$$

Let $P \rightarrow X$ be a conservative family of geometric points. For a $\Lambda$-sheaf $\mathcal{F}$ on $X$, let $\mathcal{F} \rightarrow \mathcal{C}(\mathcal{F})$ be the Godement resolution defined by $P(\underline{5}$, 4.2.2). A canonical map $\mathcal{F} \otimes i_{*} i \mathfrak{C}(\Lambda) \rightarrow i_{*} i \mathfrak{C}(\mathcal{F})$ is defined as follows. Let $U \rightarrow X$ be an etale morphism and $s \in \mathcal{F}(U)$ be a local section. Then, by regarding $s$ as a map $\left.\Lambda_{U} \rightarrow \mathcal{F}\right|_{U}$, we obtain a map $s:\left.\left.i_{*} i ! \mathcal{C}(\Lambda)\right|_{U} \rightarrow i_{*} i \mathfrak{C}(\mathcal{F})\right|_{U}$ by functoriality. In other words, we have a map $\left.\left.\mathcal{F}(U) \otimes i_{*} i^{!} \mathcal{C}(\Lambda)\right|_{U} \rightarrow i_{*} i^{!} \mathcal{C}(\mathcal{F})\right|_{U}$. They define a map $\mathcal{F} \otimes i_{*} i^{!} \mathcal{C}(\Lambda) \rightarrow i_{*} i^{\prime} \mathcal{C}(\mathcal{F})$ or equivalently $i^{*} \mathcal{F} \otimes i^{!} \mathcal{C}(\Lambda) \rightarrow i ! \mathcal{C}(\mathcal{F})$.

Let $\mathcal{K}$ be an object of $D^{-}(X, \Lambda)$. Let $\mathcal{K}_{1} \rightarrow \mathcal{K}$ be the Cartan-Eilenberg flat resolution. The map defined above induces a map $a: i^{*} \mathcal{K}_{1} \otimes i^{!} \mathcal{C}(\Lambda) \rightarrow i^{!} \mathcal{C}\left(\mathcal{K}_{1}\right)$ of double complexes. Let $N>0$ be an integer greater than the cohomological dimension of $i^{!}$. Let $\tau_{<N}^{\prime \prime}$ be the partial canonical truncation with respect to the Godement degree. Then we define

$$
\Delta_{i}(\mathcal{K})=\text { Cone }\left(\int\left(i^{*} \mathcal{K}_{1} \otimes i^{!} \tau_{<N}^{\prime \prime} \mathcal{C}(\Lambda)\right) \rightarrow \int i^{!} \tau_{<N}^{\prime \prime} \mathcal{C}\left(\mathcal{K}_{1}\right)\right)
$$

to be the mapping cone of the map of simple complexes associated to the partial truncation of the map of double complexes.

Lemma 5.1.1 For $\mathcal{K} \in D^{-}(X, \Lambda)$, we have a distinguished triangle

$$
\rightarrow i^{*} \mathcal{F} \otimes \otimes^{L} R i^{!} \Lambda \rightarrow R i^{!} \mathcal{F} \rightarrow \Delta_{i} \mathcal{F} \rightarrow .
$$

Proof. The simple complexes associated to $i^{*} \mathcal{K}_{1} \otimes i^{!} \tau_{<N}^{\prime \prime} \mathcal{C}(\Lambda)$ and $i^{!} \tau_{<N}^{\prime \prime} \mathcal{C}\left(\mathcal{K}_{1}\right)$ compute $i^{*} \mathcal{F} \otimes^{L} R i ! \Lambda$ and $R i^{!} \mathcal{F}$ respectively. Further the map a defines the canonical map $i^{*} \mathcal{F} \otimes{ }^{L} R i^{!} \Lambda \rightarrow R i ! \mathcal{F}$. Hence, the assertion follows.

Corollary 5.1.2 If the cohomology sheaves $\mathcal{H}^{q} \mathcal{K}$ are smooth on a open subscheme $U \subset X$, the complex $\Delta_{i} \mathcal{K}$ is acyclic on $Z \cap U$.

Proof. The canonical map $i^{*} \mathcal{F} \otimes^{L} R i^{!} \Lambda \rightarrow R i^{!} \mathcal{F}$ is an isomorphism on $U \cap Z$. Hence, the assertion follows from the distinguished triangle (5.1). 


\subsection{Localized characteristic class}

Let $X$ be a scheme over $k$ and $\delta: X \rightarrow X \times X$ be the diagonal map. Let $\mathcal{F}$ be an object of $D_{\text {ctf }}(X)$ and put $\mathcal{H}=R \mathcal{H} o m\left(\operatorname{pr}_{1}^{*} \mathcal{F}, \operatorname{pr}_{2}^{!} \mathcal{F}\right)$ on $X \times X$. Recall that the characteristic class $C(\mathcal{F}) \in H^{0}\left(X, \mathcal{K}_{X}\right)$ is defined by the composition $\delta_{*} \Lambda \rightarrow \mathcal{H} \rightarrow \delta_{*} \mathcal{K}_{X}$ by Proposition 2.1.7. We consider

$$
\Delta_{\delta} \delta_{*} \Lambda \rightarrow \Delta_{\delta} \mathcal{H} \rightarrow \Delta_{\delta} \delta_{*} \mathcal{K}_{X}
$$

Assume the cohomology sheaves $\mathcal{H}^{q} \mathcal{F}$ are smooth on the complement $U$ of a closed subscheme $S \subset X$. Then, the cohomology sheaves of $\mathcal{H}$ are smooth on $U \times U$ and hence the complex $\Delta_{\delta} \mathcal{H}$ on the diagonal $X$ is acyclic on $U$ by Corollary 5.1.2. Hence the composition of the canonical map $\Lambda_{X}=R \delta^{!} \delta_{*} \Lambda_{X} \rightarrow \Delta_{\delta} \delta_{*} \Lambda$ with the composition of (15.2) defines a cohomology class $C_{S}^{0}(\mathcal{F}) \in H_{S}^{0}\left(X, \Delta_{\delta} \delta_{*} \mathcal{K}_{X}\right)$.

Further assume $X$ is smooth of dimension $d$. Since the diagonal $\delta: X \rightarrow X \times X$ is a section of the smooth projection $\mathrm{pr}_{2}: X \times X \rightarrow X$, we have an isomorphism $\mathcal{K}_{X} \otimes R \delta^{!} \Lambda \rightarrow \Lambda_{X}$. Hence, by Corollary 5.1.2, we obtain a distinguished triangle

$$
\rightarrow \Lambda \rightarrow \mathcal{K}_{X} \rightarrow \Delta_{\delta} \delta_{*} \mathcal{K}_{X} \rightarrow
$$

and hence an exact sequence

$$
H_{S}^{0}(X, \Lambda) \rightarrow H_{S}^{0}\left(X, \mathcal{K}_{X}\right) \rightarrow H_{S}^{0}\left(X, \Delta_{\delta} \delta_{*} \mathcal{K}_{X}\right) \rightarrow H_{S}^{1}(X, \Lambda)
$$

We also assume that $U=X \backslash S$ is dense in $X$. Then, we have $H_{S}^{0}(X, \Lambda)=H_{S}^{1}(X, \Lambda)=0$ and hence the canonical map $H_{S}^{0}\left(X, \mathcal{K}_{X}\right) \rightarrow H_{S}^{0}\left(X, \Delta_{\delta} \delta_{*} \mathcal{K}_{X}\right)$ is an isomorphism. Thus, in this case, the class $C_{S}^{0}(\mathcal{F}) \in H_{S}^{0}\left(X, \Delta_{\delta} \delta_{*} \mathcal{K}_{X}\right)$ gives an element of $H_{S}^{0}\left(X, \mathcal{K}_{X}\right)$.

Definition 5.2.1 Let $X$ be a smooth scheme over $k$ and $S \subset X$ be a closed subscheme such that the complement $U$ is dense. Let $\mathcal{F}$ be an object of $D_{\text {ctf }}(X)$ and assume that the cohomology sheaves $\mathcal{H}^{q} \mathcal{F}$ are smooth on $U$. We call $C_{S}^{0}(\mathcal{F}) \in H_{S}^{0}\left(X, \mathcal{K}_{X}\right)$ the localized characteristic class of $\mathcal{F}$.

Lemma 5.2.2 Let $X$ be a smooth scheme over $k$ and $\left(\mathcal{F}, F_{\bullet}\right)$ be an object of $D F_{\text {ctf }}(X)$. Assume that the cohomology sheaves $\mathcal{H}^{q} G r_{p}^{F} \mathcal{F}$ are smooth on the complement of a closed subscheme $S \subset X$ for each $p \in \mathbb{Z}$. Then, we have

$$
C_{S}^{0}(\mathcal{F})=\sum_{q} C_{S}^{0}\left(G r_{q}^{F} \mathcal{F}\right)
$$

in $H_{S}^{0}\left(X, \mathcal{K}_{X}\right)$

Proof. The proof is similar to that of Lemma 2.1.3. The map $\delta_{*} \Lambda \rightarrow \mathcal{H}=$ $R \mathcal{H}$ om $\left(\operatorname{pr}_{2}^{*} \mathcal{F}, \operatorname{pr}_{1}^{!} \mathcal{F}\right)$ is induced by a map $\delta_{*} \Lambda \rightarrow F_{0} \mathcal{H}=F_{0} R \mathcal{H} o m\left(\operatorname{pr}_{2}^{*} \mathcal{F}, \operatorname{pr}_{1}^{!} \mathcal{F}\right)$. We have a canonical isomorphism $G r_{0}^{F} \mathcal{H} \rightarrow \bigoplus_{q} R \mathcal{H}$ om $\left(\operatorname{pr}_{2}^{*} G r_{q}^{F} \mathcal{F}, \operatorname{pr}_{1}^{!} G r_{q}^{F} \mathcal{F}\right)$. The map $\mathcal{H} \rightarrow \delta ! \mathcal{K}_{X}$ is induced by the sum of the maps $\mathcal{H}_{q}=R \mathcal{H} o m\left(\operatorname{pr}_{2}^{*} G r_{q}^{F} \mathcal{F}, \operatorname{pr}_{1}^{!} G r_{q}^{F} \mathcal{F}\right) \rightarrow$ 
$\delta ! \mathcal{K}_{X}$. Thus the composition $\delta_{*} \Lambda \rightarrow F_{0} \mathcal{H} \rightarrow \mathcal{H} \rightarrow \delta ! \mathcal{K}_{X}$ is equal to the sum of the compositions $\delta_{*} \Lambda \rightarrow F_{0} \mathcal{H} \rightarrow \mathcal{H}_{q} \rightarrow \delta^{!} \mathcal{K}_{X}$. Since the cohomology sheaves of $F_{0} \mathcal{H}$ are smooth on $U \times U$, the assertion follows.

We show that the localized characteristic class refines the characteristic class of $\mathcal{F}$.

Proposition 5.2.3 Let $X$ be a smooth scheme over $k$ and $S \subset X$ be a closed subscheme such that the complement $U$ is dense. Let $\mathcal{F}$ be an object of $D_{\mathrm{ctf}}(X)$ and assume that the cohomology sheaves $\mathcal{H}^{q} \mathcal{F}$ are smooth on $U$. Then the image of the localized characteristic class $C_{S}^{0}(\mathcal{F}) \in H_{S}^{0}\left(X, \mathcal{K}_{X}\right)$ by the canonical map $H_{S}^{0}\left(X, \mathcal{K}_{X}\right) \rightarrow$ $H^{0}\left(X, \mathcal{K}_{X}\right)$ is equal to the difference $C(\mathcal{F})-\left.\operatorname{rank} \mathcal{F}\right|_{U} \cdot C(\Lambda)$.

Proof. Let $j: U \rightarrow X$ be the open immersion. By applying Lemma 5.2.2 to the filtration defined by the subcomplex $j_{!} \mathcal{F}_{U} \subset \mathcal{F}$, it is reduced to the cases where $\mathcal{F}_{U}=0$ and $\mathcal{F}=j ! \mathcal{F}_{U}$ respectively.

First, we show the case $\mathcal{F}_{U}=0$. In this case, the complex $\mathcal{H}$ is supported on $S \times S$ and the localized class $C_{S}^{0}(\mathcal{F}) \in H_{S}^{0}\left(X, \mathcal{K}_{X}\right)$ is defined by the composition $\delta_{*} \Lambda \rightarrow \mathcal{H} \rightarrow \delta_{*} \mathcal{K}_{X} \rightarrow \delta_{*} \Delta_{\delta} \delta_{*} \mathcal{K}_{X}$. Thus the assertion follows in this case.

We show the case $\mathcal{F}=j_{!} \mathcal{F}_{U}$. First, we consider the case $\mathcal{F}=j_{!} \Lambda$. By Corollary 5.1.2 we have $C_{S}^{0}(\Lambda)=0$. Hence we have $C_{S}^{0}\left(j_{!} \Lambda\right)=-C_{S}^{0}\left(i_{*} \Lambda\right)$ by Lemma 5.2 .2 and its image is $-C\left(i_{*} \Lambda\right)=C\left(j_{!} \Lambda\right)-C(\Lambda)$. Thus the assertion is proved in this case.

We prove the general case $\mathcal{F}=j_{!} \mathcal{F}_{U}$. Since it has been proved for $j_{!} \Lambda_{U}$, it suffices to show that the image of $C_{S}^{0}\left(j_{!} \mathcal{F}\right)-\operatorname{rank} \mathcal{F} \cdot C_{S}^{0}\left(j_{!} \Lambda\right)$ is equal to $C\left(j_{!} \mathcal{F}\right)-\operatorname{rank} \mathcal{F} \cdot C\left(j_{!} \Lambda\right)$. To show this, we define a variant $C_{S !}^{0}\left(j ! \mathcal{F}_{U}\right) \in H_{S}^{0}\left(X, \Delta_{\delta} \delta_{*} j_{!} \mathcal{K}_{U}\right)$ of the difference $C_{!}\left(j ! \mathcal{F}_{U}\right)-$ rank $\mathcal{F} \cdot C_{!}\left(j_{!} \mathcal{F}_{U}\right) \in H^{0}\left(X, j_{!} \mathcal{K}_{U}\right)$. Recall that the refinement $C_{!}\left(j_{!} \mathcal{F}_{U}\right)$ of $C\left(j_{!} \mathcal{F}_{U}\right)$ is defined in Definition 2.1 .8 by the composition of $\delta_{*} \Lambda \rightarrow \mathcal{H} \rightarrow \delta_{*} j_{!} \mathcal{K}_{U}$. Applying $\Delta_{\delta}$ to this, we obtain a cohomology class $C_{S !}^{0}\left(j_{!} \mathcal{F}_{U}\right) \in H_{S}^{0}\left(X, \Delta_{\delta} \delta_{*} j_{!} \mathcal{K}_{U}\right)$. Since its image in $H_{S}^{0}\left(X, \Delta_{\delta} \delta_{*} \mathcal{K}_{X}\right) \stackrel{\sim}{\leftarrow} H_{S}^{0}\left(X, \mathcal{K}_{X}\right)$ is $C_{S}^{0}\left(j ! \mathcal{F}_{U}\right)$, it suffices to show the following.

Lemma 5.2.4 1. The difference $C_{S !}^{0}\left(j_{!} \mathcal{F}_{U}\right)-\operatorname{rank} \mathcal{F} \cdot C_{S !}^{0}\left(j_{!} \Lambda\right)$ is in the image of the injection $H_{S}^{0}\left(X, j_{!} \mathcal{K}_{U}\right) \rightarrow H_{S}^{0}\left(X, \Delta_{\delta} \delta_{*} j_{!} \mathcal{K}_{U}\right)$.

2. The image of its inverse image in $H_{S}^{0}\left(X, j_{!} \mathcal{K}_{U}\right)$ by the map $H_{S}^{0}\left(X, j_{!} \mathcal{K}_{U}\right) \rightarrow$ $H^{0}\left(X, j_{!} \mathcal{K}_{U}\right)$ is equal to the difference $C_{!}\left(j_{!} \mathcal{F}\right)-\operatorname{rank} \mathcal{F} \cdot C_{!}\left(j_{!} \Lambda\right)$.

Proof. We may assume $X$ and hence $U$ is connected. We may also assume $S$ is nonempty. We consider a commutative diagram

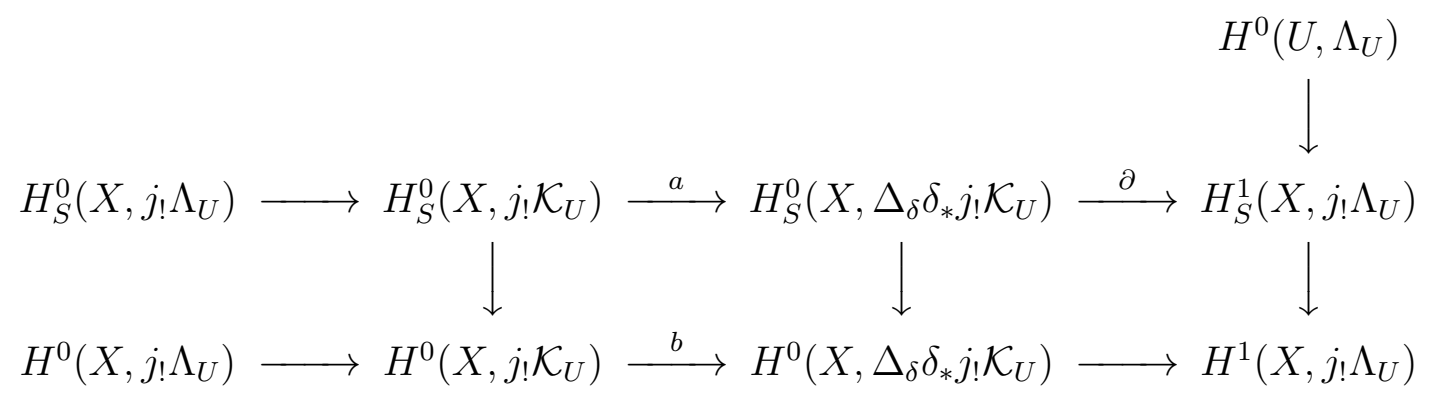


of exact sequences. The horizontal arrows are induced by the distinguished triangle $\rightarrow j_{!} \Lambda_{U} \rightarrow j_{!} \mathcal{K}_{U} \rightarrow \Delta_{\delta} \delta_{*} j_{!} \mathcal{K}_{U} \rightarrow$, similar to (5.3). The classes $C_{S !}^{0}\left(j_{!} \mathcal{F}\right)$ and $C_{S !}^{0}\left(j_{!} \Lambda\right)$ lie in third term $H_{S}^{0}\left(X, \Delta_{\delta} \delta_{*} j_{!} \mathcal{K}_{U}\right)$ on the upper line and $C_{!}\left(j_{!} \mathcal{F}\right)$ and $C_{!}\left(j_{!} \Lambda\right)$ lie in the second term $H^{0}\left(X, j_{!} \mathcal{K}_{U}\right)$ on the lower line. By the definition, they have the same images in the third term $H^{0}\left(X, \Delta_{\delta} \delta_{*} j_{!} \mathcal{K}_{U}\right)$ on the lower line.

Since $H_{S}^{0}\left(X, j_{!} \Lambda_{U}\right)=H^{0}\left(X, j_{!} \Lambda_{U}\right)=0$, the horizontal arrows $a$ and $b$ are injective. Thus, it suffices to prove

$$
\partial C_{S !}^{0}(j ! \mathcal{F})=\operatorname{rank} \mathcal{F} \cdot \partial C_{S !}^{0}(j ! \Lambda)
$$

in $H_{S}^{1}\left(X, j_{!} \Lambda_{U}\right)$.

By the exact sequence $0=H_{S}^{0}\left(X, \Lambda_{X}\right) \rightarrow H_{S}^{0}\left(X, \Lambda_{S}\right) \rightarrow H_{S}^{1}\left(X, j_{!} \Lambda_{U}\right) \rightarrow H_{S}^{1}\left(X, \Lambda_{X}\right)$ $=0$, the map $H^{0}\left(S, \Lambda_{S}\right)=H_{S}^{0}\left(X, \Lambda_{S}\right) \rightarrow H_{S}^{1}\left(X, j_{!} \Lambda_{U}\right)$ is an isomorphism. Thus, we may regard the equality (5.4) as an equality in $H^{0}(S, \Lambda)$.

By the commutative diagram, the boundary $\partial C_{S !}^{0}(j ! \mathcal{F})$ is in the image of the injection $H^{0}\left(X, \Lambda_{X}\right)=H^{0}\left(U, \Lambda_{U}\right) \rightarrow H_{S}^{1}\left(X, j_{!} \Lambda_{U}\right)=H^{0}\left(S, \Lambda_{S}\right)$. Thus, we may further regard the equality (5.4) as an equality in $H^{0}\left(X, \Lambda_{X}\right)$.

We show the equality (5.4) in $H^{0}\left(X, \Lambda_{X}\right)$, by reducing it to the case where $\mathcal{F}$ is unramified along $S$. Take a closed point $x \in U$ and put $S^{\prime}=S \amalg\{x\}$ and $U^{\prime}=$ $X \backslash S^{\prime}$. Let $j^{\prime}: U^{\prime} \rightarrow X$ be the open immersion and put $\mathcal{F}^{\prime}=\left.\mathcal{F}\right|_{U^{\prime}}$. Then, in the direct sum decomposition $H^{0}\left(S^{\prime}, \Lambda\right)=H^{0}(S, \Lambda) \oplus H^{0}(x, \Lambda)$, we have $C_{S^{\prime} !}^{0}\left(j_{!}^{\prime} \mathcal{F}^{\prime}\right)=$ $\left(\partial C_{S !}^{0}(j ! \mathcal{F}), \partial C_{x !}^{0}\left(j_{!}^{\prime} \mathcal{F}\right)\right) \in H^{0}(S, \Lambda) \oplus H^{0}(x, \Lambda)$. It lies in the diagonal image of $H^{0}(X, \Lambda)$. Since the construction is étale local, we have $\partial C_{x !}^{0}\left(j_{!}^{\prime} \mathcal{F}\right)=\operatorname{rank} \mathcal{F} \cdot \partial C_{x !}^{0}\left(j_{!}^{\prime} \Lambda\right)$. Thus we also have $\partial C_{S !}^{0}\left(j_{!} \mathcal{F}\right)=\operatorname{rank} \mathcal{F} \cdot \partial C_{S !}^{0}\left(j_{!} \Lambda\right)$ and the assertion is proved.

\section{References}

[1] A. Abbes and T. Saito, Ramification of local fields with imperfect residue fields I, Americal J. of Mathematics, 124.5 (2002), 879-920, ibid. II, Documenta Mathematica, Extra Volume Kato (2003), 3-70.

[2] —- Analyse micro-locale $\ell$-adique en caractéristique $p>0$. Le cas d'un trait, (preprint) math.AG/0602285.

[3] J.-L. Brylinski, Théorie du corps de classes de Kato et revêtements abéliens de surface, Ann. Inst. Fourier 33 (1983), 23-38.

[4] A. J. de Jong, Families of curves and alterations, Ann. Inst. Fourier (Grenoble) 47 (1997), no. 2, 599-621.

[5] P. Deligne, Cohomologie à supports propre, exposé XVII, SGA 4 tome 3, Springer LNM 305 (1973) 250-480.

[6] ——, La formule de dualité globale, exposé XVIII, SGA 4 tome 3, Springer LNM 305 (1973) 481-587. 


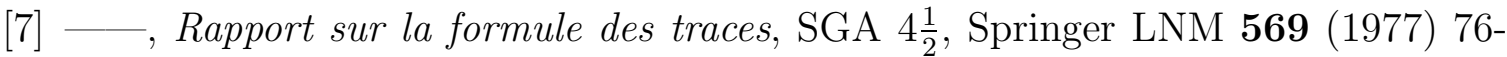
109.

[8] G. Faltings, Crystalline cohomology and p-adic Galois-representations, Algebraic analysis, geometry, and number theory (Baltimore, MD, 1988), 25-80, Johns Hopkins Univ. Press, Baltimore, MD, 1989.

[9] D. Ferrand, On the non additivity of the trace in derived categories, (preprint) math.CT/0506589.

[10] W. Fulton, Intersection theory, 2nd ed. Ergeb. der Math. und ihrer Grenz. 3. Folge. 2, Springer-Verlag, Berlin, 1998.

[11] A. Grothendieck, rédigé par L. Illusie, Formule de Lefschetz, exposé III, SGA 5, Springer LNM 589 (1977) 73-137.

[12] L. Illusie Complexes cotangent et déformations I, Springer LNM 239 (1971).

[13] M. Kashiwara, P. Schapira, Sheaves on manifolds, A Series of comprehensive studies in Mathematics 292 (1990), Springer-Verlag.

[14] K. Kato, Swan conductors for characters of degree one in the imperfect residue field case, Algebraic K-theory and algebraic number theory (Honolulu, HI, 1987), 101-131, Contemp. Math., 83, Amer. Math. Soc., Providence, RI, 1989.

[15] — Class field theory, $\mathcal{D}$-modules, and ramification of higher dimensional schemes, Part I, American J. of Math., 116 (1994), 757-784.

[16] K. Kato, T. Saito, Ramification theory of schemes over a perfect field, (preprint) math.AG/0402010 (to appear in Ann. of Math.).

[17] R. Pink, On the calculation of local terms in the Lefschetz-Verdier trace formula and its application to a conjecture of Deligne, Ann. of Math. (2) 135 (1992), no. $3,483-525$.

[18] Mme M. Raynaud (d'après notes inédites de A. Grothendieck), Propreté cohomologique des faisceaux d'ensembles et des faisceaux de groupes non commutatifs, exposé XIII, SGA 1, Springer LNM 224 (1971), Édition recomposée SMF (2003).
Ahmed Abbes
Takeshi Saito
LAGA,
Department of Mathematical Sciences,
Université de Paris 13,
University of Tokyo,
99, Av. J.-B. Clément,
Tokyo 153-8914 Japan
93430 Villetaneuse France
abbes@math.univ-paris13.fr
t-saito@ms.u-tokyo.ac.jp 